\title{
PHYLOGENETIC ANALYSES OF POSTCRANIAL SKELETAL MORPHOLOGY IN DIDELPHID MARSUPIALS
}

\author{
DAVID A. FLORES \\ Museo Argentino de Ciencias Naturales "Bernardino Rivadavia" \\ Buenos Aires, Argentina \\ (dflores@macn.gov.ar)
}

BULLETIN OF THE AMERICAN MUSEUM OF NATURAL HISTORY CENTRAL PARK WEST AT 79TH STREET, NEW YORK, NY 10024 Number 320, 81 pp., 40 figures, 8 tables Issued March 17, 2009 


\section{CONTENTS}

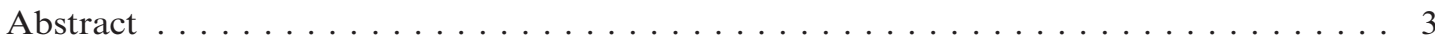

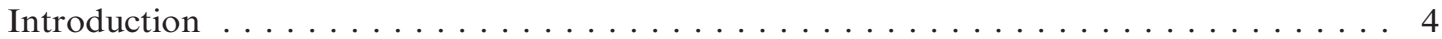

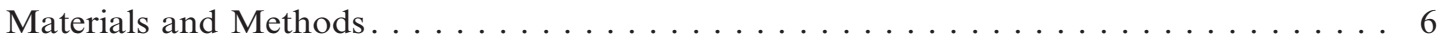

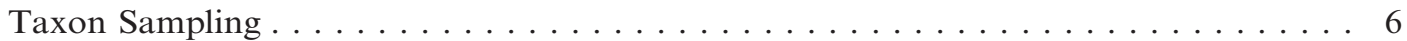

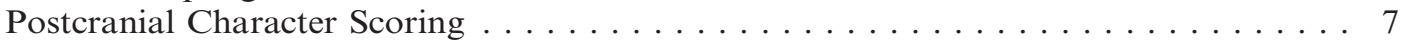

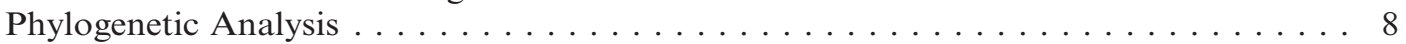

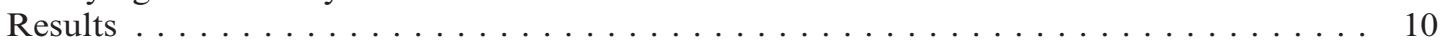

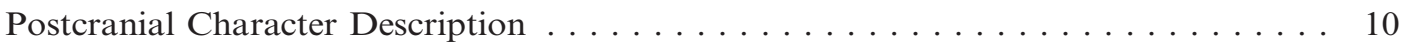

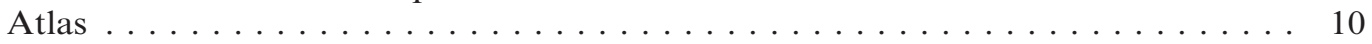

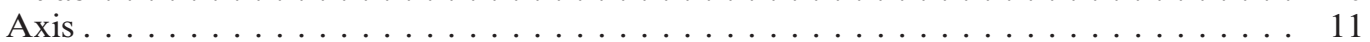

Posterior Cervical Vertebrae. . . . . . . . . . . . . . . . . . . . . . . 13

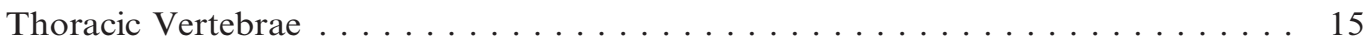

Lumbar Vertebrae . . . . . . . . . . . . . . . . . . . . . . . . . . . . . . 19

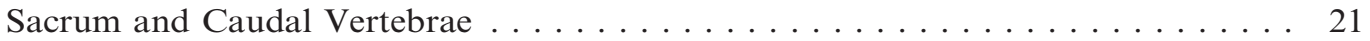

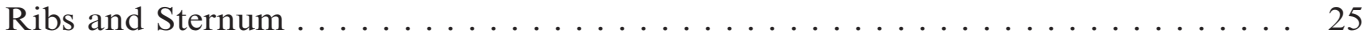

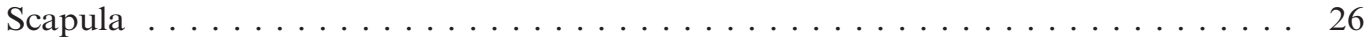

Humerus. . . . . . . . . . . . . . . . . . . . . . . . . 29

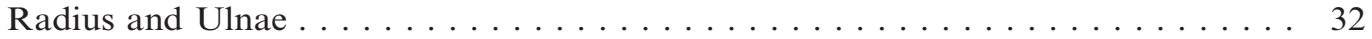

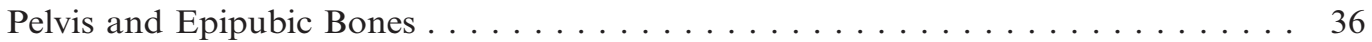

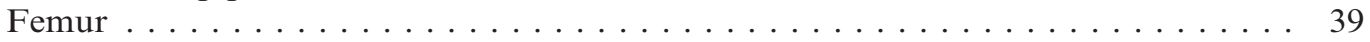

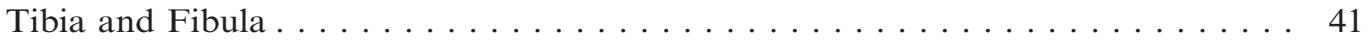

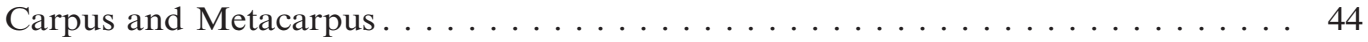

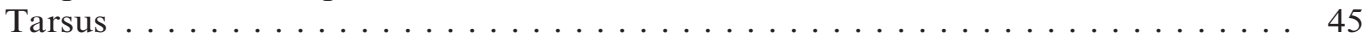

Postcranial Data Set Summary. . . . . . . . . . . . . . . . . . 47

Analytic Results of Postcranial Characters . . . . . . . . . . . . . . . . . . 47

Nonmolecular Evidence on Didelphid Phylogeny: The Inclusion of Postcranial

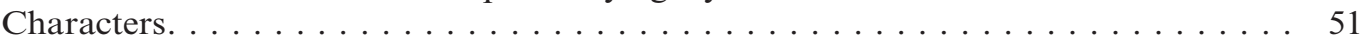

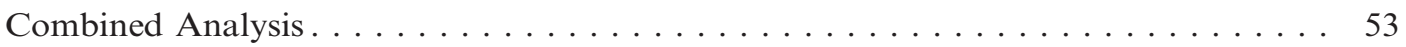

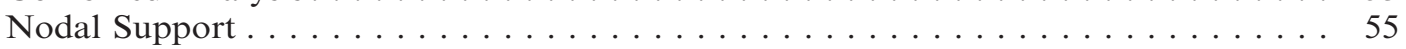

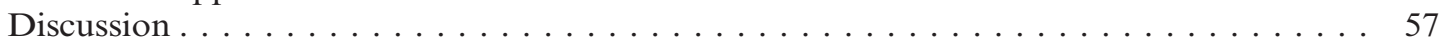

Effects of Different Codings of Polymorphic Data . . . . . . . . . . . . . . 57

Effect of the Inclusion of Postcranial Characters in Previous Nonmolecular and Combined Hypotheses . . . . . . . . . . . . . . . . . . . . . . . 59

The Postcranial Anatomy as Evidence of Didelphid Relationships and

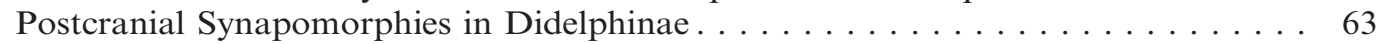

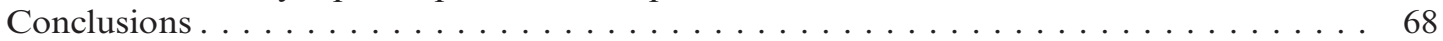

Acknowledgments . . . . . . . . . . . . . . . . . . . . . . . . . . 69

References . . . . . . . . . . . . . . . . . . . . . . . . . . . 69

Appendix 1: Postcranial Material Examined . . . . . . . . . . . . . . . 74

Appendix 2: Postcranial Data Matrix . . . . . . . . . . . . . . . . . . . 74

Appendix 3: Nodal Synapomorphies in Strict Consensus of Combined Hypotheses . . . 76 


\begin{abstract}
In this study I provide a phylogenetic hypothesis for didelphid Marsupials including a suite of 114 postcranial characters. The postcranial evidence was cladistically analyzed separately and concatenated with a nonmolecular data set previously published (71 cranio-dental-external characters). A combined analysis was done including published IRBP (interphotoreceptor retinoid binding protein, $1158 \mathrm{bp}$ ), DMP-1 (dentin matrix protein 1, $1176 \mathrm{bp}$ ), and RAG-1 (recombinase-activating gene, $2790 \mathrm{bp}$ ) sequences to the nonmolecular data set. In order to compare and evaluate the influence of the inclusion of postcranial morphology to previous hypotheses, the taxon sampling of didelphine ingroup was almost similar to the one used in recent series of papers on didelphid phylogeny. The postcranial information includes 48 characters from the axial skeleton, 37 from the anterior limb, and 29 from the posterior limb. I present anatomical descriptions for each postcranial character, adding details of different conditions observed among didelphine ingroup, as well as some functional implications. Different hypotheses that are discussed as polymorphic characters are alternatively treated as composite entries (CO) and transformation series (TS) in morphological and combined analyses. Different codings of polymorphic postcranial characters produce topologies that in general are not contradictory. The principal difference is the loss of resolution of trees in TS analysis, compared to $\mathrm{CO}$ analysis in postcranial evidence, whereas the support values were in general low in both codings. The topology obtained from postcranial evidence supported some already recovered relationships, such as the monophyly of the large opossums (Didelphis, Philander, Chironectes, Lutreolina, and Metachirus), and several polytypic groups such as Didelphis, Monodelphis, Marmosops, Thylamys, Micoureus, and Philander. Additionally, the intermediate position of Hyladelphys between calorumyines and didelphines is kept in $\mathrm{CO}$ analysis. The inclusion of the postcranial data set to previous nonmolecular evidence causes little incongruence, although some modifications in the topology and support values were detected. The effect of different codings of polymorphic characters was similar respect to the postcranialonly data set. In this case, the topology obtained with $\mathrm{CO}$ analysis was also notably better resolved than TS analysis. Similarly to the postcranial-only analysis, the topologies obtained in the total morphological evidence applying the two kinds of codings are highly congruent, but the TS treatment seemed not to contribute to retention of more phylogenetic information, since the $\mathrm{CO}$ analysis was better resolved. The relationships obtained adding the postcranial evidence to published combined data set (i.e., morphology, IRBP, DMP-1, and RAG-1 sequences) were mostly better resolved and supported in the CO coding than the morphological analyses, although the TS coding causes loss of resolution in the strict consensus. In this sense, some strong differences on deep branch topology can be detected depending on the treatment applied to polymorphic entries and partitioned analyses (e.g., phylogenetic condition of the mouse opossums, nodes $\mathrm{C}$ and $\mathrm{B}$ ). Including the postcranial evidence in the total data set, I also recovered the intermediate position of Hyladelphys, but never the distantly related clades recently recovered by the inclusion of RAG-1 sequences (clades B + I in Gruber, K.F., R.S. Voss, and S.A. Jansa. 2007. Base-compositional heterogeneity in the RAG1 locus among didelphid marsupials: implications for phylogenetic inference and the evolution of GC content. Systematic Biology 56: 1-14). However, the position of Metachirus nudicaudatus and Tlacuatzin canescens is highly affected. Excluding the last gene, the partitioned combined analysis considering postcranial evidence was highly congruent with previous IRBP, morphology, and combined topologies, especially in the TS analysis, where all already defined nodes were recovered. The inclusion of postcranial evidence to the previous combined data set actually improves the support values when RAG-1 is eliminated. However, even when the topologies from both kinds of codings of polymorphic data were considerably congruent, the mouse opossums were clustered in CO analysis (although including Metachirus if RAG-1 sequences are included). The application of different criteria for the treatment of polymorphic data affects the relationships and phyletic condition of the mouse opossums. Finally, I discuss the postcranial morphology as evidence of didelphid phylogeny, as well as the new postcranial synapomorphies found in the monophyletic groups recovered in the didelphid crown group.
\end{abstract}




\section{INTRODUCTION}

Didelphid marsupials represent a living Neotropical radiation including around 85 species (Gardner, 2005) ranging from Canada to Patagonia. Through the past decades, the study of the New World living marsupials has been focused in diverse disciplines, resulting in an important amount of information available, ranging from systematics and taxonomy to anatomy, ontogeny, behavior, ecology, biogeography, physiology, and cytogenetics. As indicated by Voss and Jansa (2003), this interesting group represents the most diverse living radiation of the old endemic fauna that evolved in South America during the Tertiary isolation, and it is the only large radiation of metatherian mammals outside of Australia (Simpson, 1971; Patterson and Pascual, 1972).

In studies of marsupial evolution, didelphids were traditionally identified as "primitive" relative to the remaining marsupial families (Bensley, 1903; Gregory, 1910) and were thought to be models to extrapolations for Paleogene and Cretaceous metatherian biology (e.g., Crompton and Hiiemae, 1970; Crompton and Hylander, 1986; Crompton, 1989). Additionally, some modern studies placed either Didelphimorphia or Ameridelphia (i.e., Didelphimorphia + Paucituberculata) as the basal branch of the marsupial clade (see Horovitz and Sánchez-Villagra, 2003; Asher et al., 2004). However, it is widely recognized at present that living didelphids are not "living fossils", and even though they are considered basal relative to most of the Australasian living marsupials, they are derived relative to most Cretaceous and Paleogene metatherians (Clemens, 1968; Szalay, 1982, 1994; Reig et al., 1987; Wible, 1990; Goin, 1993; Horovitz and SánchezVillagra, 2003; Sánchez-Villagra et al., 2007).

In a morphological and distributional framework, didelphids have been traditionally diagnosed by plesiomorphies and biogeographical patterns, and some studies have demonstrated the failures of morphological characters to recover didelphids as a monophyletic group (see Kirsch and Archer, 1982; Wroe et al., 2000). The phylogenetic study by Horovitz and Sánchez-Villagra (2003) provides some unambiguous and unreversed postcranial synapomorphies for Didelphidae (e.g., calcaneal notch for cuboid present, or ridge on proximal articular facet of metacarpal I present). However, only two taxa representing the whole family were considered in that paper (Didelphis and Monodelphis), as the main scope was focused on a high-level marsupial relationship. In this sense, a more inclusive taxon sampling is neccesary for scoring most postcranial characters described in didelphids, detecting phylogenetic information in a family-level context as well. The same lack of a denser taxon sampling was noted for other morphological characters such as sperm morphology and mammary pattern (Jansa and Voss, 2005). On the contrary, IRBP (interphotoreceptor retinoid binding protein, $1158 \mathrm{bp}$ ) sequences provide strong evidence of didelphid monophyly, since the family is supported for 10 uniquely derived and unreversed transformations (Jansa and Voss, 2000, 2005) in the context of a higher taxonomic sample.

The study of the phylogenetic relationships in didelphids has been based on different points of view, resulting in occasionally wellsupported hypotheses with evidence from diverse sources. For instance, the monophyly of the large $2 n=22$ opossums was recovered using different kinds of data (e.g. Creighton, 1984; Reig et al., 1987; Kirsch and Palma, 1995; Jansa and Voss, 2000, 2005; Flores, 2003; Voss and Jansa, 2003; Gruber et al., 2007), whereas in other cases there were conflicting patterns, as the paraphyletic condition of some genera of mouse opossums, currently considered as taxonomically valid (see Gardner, 2005). According to Voss and Jansa (2003), the incongruence among different hypotheses is likely caused by several factors, such as disparate taxon sampling, use of nonmonophyletic supraspecific taxa as terminals, discrepant scoring of the same character, different rooting criteria and algorithms, and voucher misidentifications.

Recently, the study of didelphid phylogeny has expanded markedly due to new knowledge and interpretations of the external and internal anatomy, new DNA sequence information from nuclear genes (e.g., IRBP, DMP-1 [dentin matrix protein 1, 1176 bp], RAG-1 [recombinase-activating gene, 2790 
bp]), inclusion of a larger taxonomic sample, and taxonomic clarification of some polytypic groups. The ingroup relationships in didelphids obtained in previous papers were partially summarized by Jansa and Voss (2000) and Voss and Jansa (2003). The molecular evidence available for a higher didelphid taxonomic sample includes sequences of different nuclear genes, such as IRBP (Jansa and Voss, 2000; 2005), DMP-1 (Jansa and Voss, 2005; Jansa et al., 2006), and RAG-1 (Gruber et al., 2007). Additionally, the morphological evidence used in a cladistic background consists of craniodental, external anatomy, and karyotypes (see Voss and Jansa, 2003 and citations therein). In relation to this diverse array of evidence, interesting hypotheses based in combined analyses were recently obtained (Voss and Jansa, 2003; Jansa and Voss, 2005; Gruber et al., 2007), whose topologies show some stability, since in some cases they are congruent, independent of the type of characters employed. Additionally, the wide taxonomic sample available made possible the formulation of more inclusive phylogenetic hypotheses. In this sense, the recent addition of new taxa such as Chacodelphys formosa (Voss et al., 2004) or Hyladelphys kalinowskii (Jansa and Voss, 2005) in some cases has modified topologies or changed the support values in different clades.

The more recent hypothesis on didelphid phylogeny based on nonmolecular characters (Jansa and Voss, 2005: fig. 1C) included craniodental, external, and karyological characters. In the topology obtained, the large $2 \mathrm{n}=22$ opossums (i.e., Didelphis, Philander, Lutreolina, and Chironectes) were recovered as a monophyletic group, closely related to the monophyletic Monodelphis and the $2 \mathrm{n}=14$ Metachirus. The mouse opossums resulted in a paraphyletic group, of which only Gracilinanus, Cryptonanus, and Marmosops were recovered as monophyletic. The position of the monotypic Chacodelphys was basal to the Lestodelphys + Thylamys group, and Hyladelphys was placed basal to the whole didelphine crown group (i.e., intermediate between caluromyines and didelphines). The combination of the morphological data set with genetic information (i.e., IRBP, DMP-1, and RAG-1 sequences) caused a better resolution on the topologies obtained with morphology alone, and the support values of most nodes had $\geq 75 \%$ bootstrap support. In this scheme, the large opossums were equally recovered, as well as several mouse opossum genera (i.e., Thylamys, Cryptonanus, Gracilinanus, Marmosops, Micoureus, and Monodelphis), and the position of Hyladelphys was similar to the results from morphological data (i.e., basal to the didelphine group, except including RAG-1 sequences). It is clear that at present the phylogenetic relationships on didelphid groups are based on profuse evidence, which in general does not exhibit substantial conflict. As stated by Jansa and Voss (2000), the node-by-node comparison of different hypotheses obtained by different sources suggested that many cases of conflict do not reflect considerable incongruence of data.

Although the study of the postcranial morphology in metatherians has received notable attention, most of it was focused on functional, ontogenetic, and descriptive frames, but not phylogenetics (e.g., Coues, 1869; Flower, 1885; Elftman, 1929; Barnett and Napier, 1953; Mann-Fischer, 1953; Jenkins and Weijs, 1979; Klima, 1987; White, 1990; Pridmore, 1992; Marshall and Sigogneau-Russell, 1995; Lunde and Schutt, 1999; Argot, 2001, 2002, 2003a, 2003b, 2004a, 2004b; Martin and Mackay, 2003; Muizon and Argot, 2003; but see Szalay, 1994; Szalay and Sargis, 2001; Horovitz and SánchezVillagra, 2003). The study of Horovitz and Sánchez-Villagra (2003) contains numerous postcranial characters treated in high-level taxonomic categories that are interpreted in a cladistic context. Some characters described in that paper have shown some variability in the ingroup considered in this report, being parsimony informative in lower taxonomic levels. Additionally, several previous papers detected notable morphological variation in the postcranial skeleton of the taxa treated, in a descriptive or functional frame. In this sense, the identification and inclusion of postcranial characters in an appropriate taxonomic sample is significant in order to be analyzed in a cladistic framework. It is essential to know the influence of inclusion of the postcranial characters, identifying con- 
gruences as well as incongruences among previous hypotheses based in other sorts of characters, and to integrate this evidence in a combined analysis to obtain hypotheses based on all available evidence of didelphid phylogeny.

In this study, I conducted a cladistic analysis of living didelphids based on postcranial characters, and I describe the postcranial character variations among 38 didelphid species, including most of those previously analyzed in other morphological frameworks (e.g., Voss and Jansa, 2003). Having considered practically the same taxonomic sample used in recent phylogenetic analyses (Voss and Jansa, 2003; Jansa and Voss, 2005; Voss et al. 2006; Jansa et al., 2006; Gruber et al., 2007) for comparative purposes, this data set was incorporated in the previous data set available (i.e., craniodental and external anatomy, karyology, plus IRBP, DMP-1, and RAG-1 sequences). I analyzed the postcranial and previously known morphological characters separately and in combination with the molecular data. I also explored the congruence and conflict between both kinds of data sets. The addition of postcranial characters to the known phylogenetic evidence is essential, since in some situations it could improve the support values on previously recognized monophyletic groups, although in other circumstances it could contradict those topologies. Furthermore, I provide additional character information in order to reach a comprehensive perception of the postcranial variation within the living didelphid group.

\section{MATERIALS AND METHODS}

\section{TAXON SAMPLING}

I analyzed the osteological postcranial morphology in 4 outgroups and 34 ingroup didelphid taxa (table 1; appendix I). In order to reach a viable comparison and combination with morphological and genetic data sets previously published in recent phylogenetic studies on didelphids (Voss and Jansa, 2003; Jansa and Voss, 2005; Jansa et al., 2006; Gruber et al., 2007), the outgroup considered is composed of a caluromyine group including Glironia venusta (root), two species of Caluromys, and Caluromysiops irrupta. The
TABLE 1

Taxonomic Diversity of Extant Didelphid Genera (Sensu Gardner, 2005, Except in Taxa Indicated) and Number of Included Species per Taxon in the Postcranial Analysis

\begin{tabular}{|c|c|c|}
\hline Genera & $\begin{array}{c}\text { No. } \\
\text { species }\end{array}$ & $\begin{array}{l}\text { No. included } \\
\text { species }\end{array}$ \\
\hline Caluromysiops & 1 & 1 \\
\hline Caluromys & 3 & 2 \\
\hline Chironectes & 1 & 1 \\
\hline Cryptonanus $^{\mathrm{a}}$ & 5 & 1 \\
\hline Didelphis & 6 & 3 \\
\hline Glironia & 1 & 1 \\
\hline Gracilinanus & 6 & 2 \\
\hline Hyladelphys & 1 & 1 \\
\hline Lestodelphys & 1 & 1 \\
\hline Lutreolina & 1 & 1 \\
\hline Marmosa & 9 & 4 \\
\hline Marmosops $^{\mathrm{b}}$ & 14 & 5 \\
\hline Metachirus & 1 & 1 \\
\hline Micoureus & 6 & 3 \\
\hline Monodelphis ${ }^{\mathrm{c}}$ & 20 & 3 \\
\hline Philander $^{\mathrm{d}}$ & 7 & 3 \\
\hline Thylamys & 10 & 4 \\
\hline Tlacuatzin & 1 & 1 \\
\hline Total & 92 & 38 \\
\hline
\end{tabular}

${ }^{\mathrm{a}}$ Genus recently recognized by Voss et al. (2006); species previously included in Gracilinanus.

${ }^{\mathrm{b}}$ Including M. creightoni (see Voss et al., 2004).

${ }^{\mathrm{c}}$ Including $M$. ronaldi (see Solari, 2004) and $M$. handleyii (see Solari, 2007).

${ }^{\mathrm{d}}$ Including $P$. mondolfii, $P$. deltai (see Lew et al., 2006), and $P$. olrogi (see Flores et al., 2008).

ingroup includes practically the same didelphine taxa as the ones in the cited papers. Postcranial samples for four species (Chacodelphys formosa, Gracilinanus emiliae, $G$. aceramarcae, Marmosa lepida, and Cryptonanus chacoensis) could not be examined in this analysis, since the material is apparently nonexistent in extant systematic collections. All polytypic groups were represented by several species in order to test the monophyletic condition of each supraspecific group (table 1). In general, I examined several adult specimens of both sexes per taxon in order to detect polymorphism (table 2). However, some species with scarce postcranial material deposited in systematic collections (e.g., Glironia venusta, Hyladelphys kalinowskii, Philander mcilhennyi, or Marmosa rubra) were represented by only one or two specimens (see appendix 1). 
TABLE 2

Polymorphic Characters Observed on Postcranial Morphology in Didelphid Taxa

(For details, see character descriptions.)

\begin{tabular}{ll}
\hline \multicolumn{1}{c}{ Taxa } & \multicolumn{1}{c}{ Character no. } \\
\hline Caluromys lanatus & 35 \\
Caluromysiops irrupta & 15,52 \\
Chironectes minimus & $35,63,87,97$ \\
Cryptonanus unduaviensis & 22 \\
Didelphis virginiana & $35,47,110$ \\
D. albiventris & $35,47,66$ \\
D. marsupialis & $35,47,59,66,110$ \\
Gracilinanus agilis & $2,17,62,63,68,77$ \\
Lutreolina crassicaudata & 47 \\
Micoureus demerarae & $18,60,70$ \\
M. regina & $17,18,20,34,88$ \\
M. paraguayanus & $1,2,59,60,62,66,70,77$ \\
Marmosops incanus & 47 \\
M. noctivagus & $2,17,35,40,57$ \\
M. pinheiroi & 40,60 \\
M. impavidus & 22,35 \\
Marmosa mexicana & 111 \\
M. robinsoni & $2,13,22,35,43,47,59,66,67,68$ \\
M. murina & $35,47,66,77$ \\
Metachirus nudicaudatus & 2,8 \\
Philander opossum & $3,47,59,102$ \\
P. frenatus & 2,87 \\
Thylamys pallidior & 22 \\
T. pusillus & $1,2,57,66$ \\
T. venustus & $4,20,22,40$ \\
Tlacuatzin canescens & 11,47 \\
\hline & \\
\hline
\end{tabular}

\section{Postcranial Character Scoring}

The parsimony analysis of didelphid species-level relationships is based on a suite of new and partially scored postcranial characters (see character descriptions and appendix 2) and the comparison and combination with the morphological data matrix previously defined by Voss and Jansa (2003). Australasian and American nondidelphid taxa were not considered part of the outgroup. Consequently, several postcranial characters that could define the didelphid position with respect to nondidelphid marsupials (see Szalay, 1994; Szalay and Sargis, 2001; Horovitz and Sánchez-Villagra, 2003) were not incorporated, as they are apparently invariant in the didelphid ingroup considered here. On the other hand, some characters described in the previous cladistic analysis of Horovitz and Sánchez-Villagra (2003) illustrated significant variation when a didelphid denser taxon sampling was included, and thus they were reanalyzed in this context of didelphid systematics. The complete morphological data set is composed of 185 characters (table 3), of which 114 are new and reanalyzed postcranial characters: 48 from the axial skeleton, 37 from anterior limbs, and 29 from posterior limbs. The remaining 71 characters were described by Voss and Jansa (2003) and include craniodental, external, and karyological evidence. In this way, the taxonomically variable postcranial characters come mainly from direct observation of the material under study and from reinterpretation of characters previously defined in studies with taxonomic, systematic, or a functional-descriptive perspective (e.g., Flower, 1885; Elftman, 1929; Barnett and Napier, 1953; Mann-Fischer, 1953; Jenkins and Weijs, 1979; Szalay, 1982, 1994; Marshall and Sigogneau-Russell, 1995; Muizon, 1998; Argot, 2001, 2002, 2003a, 2003b, 2004a, 2004b; Szalay and Sargis, 2001; Horovitz and Sánchez-Villagra, 2003; Muizon and Argot, 2003).

In a few cases, postcranial elements were incomplete, destroyed, or in bad condition. In such cases, published descriptions or drawings were consulted to assess the correct character state. Due to the absence of information in some cases, I scored the relevant matrix cells as missing ("?"). Those that resulted from unsuitable comparisons (table 4) were scored as inapplicable ("-").

In the postcranial data matrix, I defined both binary and multistate characters, where three or more alternative conditions were observed. In some cases, multistate characters could be logically ordered in a transformation series, but in other situations no compelling justification for ordering could be found (see the character descriptions). In the analyses, I included both parsimony informative characters and few autapomorphies (table 3). The latter were not eliminated because of reasons mentioned by Voss and Jansa (2003); that is, scoring autapomorphies is required to preserve the homologies in unordered multistate characters that contain parsimony informative characters, such scoring facilitates the recovery of taxon diagnoses from the data matrix, and some autapomorphies can be parsimony informative in a 
TABLE 3

Comparison of Three Data Set Characteristics of Nonmolecular Data Matrix (See text for explanation of combined nonmolecular data set.)

\begin{tabular}{lccc}
\hline \hline & Jansa and Voss (2005) & Postcranial & Combined nonmolecular data set \\
\hline No. terminal taxa ${ }^{\mathrm{a}}$ & 44 & 38 & 44 \\
No. cells & 3124 & 4332 & 8140 \\
Missing & $109(3 \%)$ & $85(2 \%)$ & $871(11 \%)$ \\
Inapplicable & $33(1 \%)$ & $44(1 \%)$ & $77(1 \%)$ \\
No. characters & 71 & 114 & 185 \\
Parsimony-informative characters & $67(94 \%)$ & $106(93 \%)$ & $173(94 \%)$ \\
Autapomorphic characters & $4(6 \%)$ & $8(7 \%)$ & $12(7 \%)$ \\
Binary characters & $53(75 \%)$ & $66(58 \%)$ & $119(64 \%)$ \\
Ordered multistate characters & $12(17 \%)$ & $47(41 \%)$ & $59(32 \%)$ \\
Unordered multistate characters & $6(8 \%)$ & $1(0.9 \%)$ & $7(4 \%)$ \\
\hline
\end{tabular}

aThe postcranial data set does not include Chacodelphys formosa, Cryptonanus chacoensis, Gracilinanus aceramarcae, G. emiliae, Marmosa lepida, and Monodelphis emiliae, but they are included in the combined nonmolecular data set (coded "?" for postcranial characters).

more denser taxon sampling and could be used in future analyses. On the other hand, to consider autapomorphies in the ingroup might eventually serve as synapomorphies for clades represented by a single taxon in the present analysis (Weksler, 2006). Proper polarity of characters was determined after rooting the trees.

The treatment of polymorphic characters was done following two methods in order to detect the influence of different criteria in the topology and support values. In the first mode, I considered the polymorphic condition as composite states (CO) in a transformation series or in a binary character (i.e., polymorphic conditions between two character states 0 and 1 , coded as $\{01\}$ for a taxon with states 0 and 1 observed among its exemplars). Alternatively, the polymorphic condition was considered as an intermediate character state in a transformation series (TS), and the series is treated as ordered (see Campbell and Frost, 1993; Wiens, 1999, 2000; Voss and Jansa, 2003; Weksler, 2006). The binary character with the polymorphic condition takes the form of a multistate character with an ordered transformation series, and the intermediate condition represents the polymorphism. With the intermediate character state representing the polymorphism, the transformations $0 \leftrightarrow 1$ and $1 \leftrightarrow 2$ each were weighed to 0.5 relative to the unit weight (1.0) assigned to the transformation 0 $\leftrightarrow 1$ in a binary character without polymor- phism. Obviously, polymorphism does not always occur in a binary character. In multistate characters, the polymorphism is included as a transitional state between two different conditions observed (whose weight is assigned with the same procedure mentioned above) so that it adds one condition to the ordered transformation series, and the multistate character is assumed as ordered.

The postcranial data matrix presented herein was composed in order to analyze the internal didelphid relationships and compare and combine with recent data sets including a denser taxon sampling (Voss and Jansa, 2003; Jansa and Voss, 2005). For this reason, it is not situable to define nondidelphid marsupial relationships. The position of didelphids and their relationships in a supraordinal context are beyond the scope of this report, but a postcranial data matrix including several nondidelphid Australasian, American, and some fossil taxa is in preparation as part of a subsequent report.

\section{Phylogenetic Analysis}

I performed parsimony analyses of postcranial evidence both separately and in combination with the nonmolecular data set (craniodental and external) devised by Jansa and Voss (2005), as well as in the supermatrix obtained by combining all the nonmolecular evidence, with IRBP, DMP-1, and RAG-1 sequences. The sequences can be aligned 
TABLE 4

Postcranial Data Completeness for Didelphid Terminal Taxa

\begin{tabular}{|c|c|c|c|}
\hline & Inapplicable & Missing & $\begin{array}{c}\% \\
\text { Complete }\end{array}$ \\
\hline Glironia venusta & 3 & 0 & 97 \\
\hline Caluromysiops irrupta & 1 & 0 & 99 \\
\hline Caluromys lanatus & 2 & 0 & 98 \\
\hline C. philander & 2 & 0 & 98 \\
\hline $\begin{array}{l}\text { Metachirus } \\
\quad \text { nudicaudatus }\end{array}$ & 0 & 0 & 100 \\
\hline Philander opossum & 1 & 0 & 99 \\
\hline P. franatus & 1 & 0 & 99 \\
\hline P. mcilhenyii & 1 & 3 & 96 \\
\hline Didelphis virginiana & 1 & 0 & 99 \\
\hline D. albiventris & 1 & 0 & 99 \\
\hline D. marsupialis & 1 & 0 & 99 \\
\hline $\begin{array}{l}\text { Lutreolina } \\
\text { crassicaudata }\end{array}$ & 2 & 3 & 96 \\
\hline Chironectes minimus & 0 & 0 & 100 \\
\hline Tlacuatzin canescens & 0 & 2 & 98 \\
\hline Thylamys pallidior & 1 & 0 & 99 \\
\hline T. pusillus & 1 & 2 & 97 \\
\hline T. venustus & 2 & 4 & 94 \\
\hline T. macrurus & 1 & 1 & 98 \\
\hline $\begin{array}{l}\text { Monodelphis } \\
\text { brevicaudata }\end{array}$ & 1 & 1 & 98 \\
\hline M. adusta & 0 & 10 & 91 \\
\hline M. theresa & 2 & 0 & 98 \\
\hline $\begin{array}{l}\text { Micoureus } \\
\quad \text { paraguayanus }\end{array}$ & 0 & 12 & 89 \\
\hline M. regina & 0 & 1 & 99 \\
\hline M. demerarae & 0 & 0 & 100 \\
\hline Marmosa rubra & 2 & 3 & 95 \\
\hline M. robinsoni & 1 & 0 & 99 \\
\hline M. mexicana & 1 & 0 & 99 \\
\hline M. murina & 0 & 3 & 97 \\
\hline Marmosops impavidus & 1 & 1 & 98 \\
\hline M. parvidens & 2 & 0 & 98 \\
\hline M. pinheiroi & 1 & 2 & 97 \\
\hline M. noctivagus & 2 & 1 & 97 \\
\hline M. incanus & 2 & 0 & 98 \\
\hline Lestodelphys halli & 1 & 13 & 88 \\
\hline $\begin{array}{l}\text { Cryptonanus } \\
\text { unduaviensis }\end{array}$ & 3 & 1 & 96 \\
\hline Gracilinanus agilis & 1 & 0 & 99 \\
\hline G. microtarsus & 1 & 13 & 88 \\
\hline $\begin{array}{l}\text { Hyladelphys } \\
\text { kalinowskii }\end{array}$ & 3 & 1 & 96 \\
\hline
\end{tabular}

unambiguously among didelphids (Jansa and Voss, 2000; Jansa et al., 2006; Grueber et al., 2007). In both morphological and combined analyses, the characters were equally weighted. All of the postcranial, total morphology, and combined matrices were analyzed with two different coding for polymorphic entries explained in the "Postcranial Character Scoring" section; that is, CO and TS analyses, respectively. For all parsimony analyses I used the program TNT (Goloboff et al., 2004) to search for optimal trees under equal weights. I conducted heuristic, unconstrained searches for optimal trees using tree bisection-reconnection (TBR) branch-swapping in each of 100 replications of random taxon addition sequences, keeping up to 10 trees per replication. A second TBR round was applied to each of the optimals in order to increase the confidence of finding all topologies of minimum length. Zero-length branches were collapsed and strict consensus trees were generated. Clade support was estimated using both absolute Bremer support (Bremer, 1994) and character resampling (Goloboff et al., 2003). Values of absolute Bremer support were calculated following the approach of Giannini and Bertelli (2004) in order to prevent overestimation of support values. Specifically, they implemented a strategy for obtaining suboptimal trees in seven successive stages in which $<2000$ suboptimal trees were saved in each stage. First, I saved suboptimal trees that were one step longer than the optimals. Next, searches saved trees up to two to seven steps longer than the optimals. Thus, in the analysis a sample of $\geq 14,000$ suboptimal trees was used to calculate absolute Bremer support. In this way, I sampled several suboptimal trees that could be overlooked in a less careful selection of suboptimals, which would lead to overestimated support values. On the other hand, the resampling technique used was a variant of the jackknife implemented by Goloboff et al. (2003). Group frequency (based on unbiased symmetric resampling) was calculated on the basis of 2000 replications.

I combined the postcranial data set with the previously defined nonmolecular characters (Voss and Jansa, 2003; Jansa and Voss, 2005). Additionally, IRBP, DMP-1, and RAG-1 sequences were included, resulting in a supermatrix analyzed using the same parsimony procedures as described above. Finally, the patterns of congruence and conflict among separate analyses (i.e., postcranial only, total morphology, and com- 
bined analysis) were evaluated by direct inspection of tree topologies and values of nodal support.

\section{RESULTS}

\section{Postcranial Character Description}

In this section I describe the variations observed on the skeletal morphology of the taxa included in the analysis. In most cases, some brief descriptions and comments about evolutionary or functional features were added to the character description, and the character state observed in the taxonomic sample is also reported. The 114 postcranial characters are listed by different skeletal sections as follows: atlas, axis, posterior cervical vertebrae, thoracic vertebrae, lumbar vertebrae, sacrum and caudal vertebrae, ribs and sternum, scapula, humerus, radius and ulna, pelvis and epipubic bones, femur, tibia and fibula, carpus and metacarpus, and tarsus. For the osteological and muscular terminology, I followed several works that treated skeletal and muscular morphology in mammals (e.g., Maynard Smith and Savage, 1955; Taylor, 1974, 1976; Evans, 1993; Gilbert, 1994; Sargis, 2002; Bezuidenbout and Evans, 2005; Whitehead et al., 2005) and specifically in marsupials (e.g. Elftman, 1929; Szalay, 1994; Marshall and Sigogneau-Russell, 1995; Muizon, 1998; Argot, 2001, 2002, 2003a, 2003b, 2004a, 2004b; Szalay and Sargis, 2001; Horovitz and Sánchez-Villagra, 2003).

\section{Atlas}

Character 1: Atlantal foramen: (0) absent; (1) present. In caluromyines and large opossums (Didelphis, Philander, Chironectes, Lutreolina, and Metachirus) the foramen is present, whereas in the mouse opossums in general this foramen is absent (except Marmosa rubra). Horovitz and Sánchez-Villagra (2003: ch. 1) reported asymmetry in the presence/absence of this character in some taxa such as Monodelphis domestica, Dasyuroides, and Dromiciops. I also found the same asymmetry in some specimens of Thylamys pusillus and Micoureus paraguayanus, and the taxa are treated as polymorphic (coded $\{01\}$ ).

Character 2: Atlas transverse foramen: (0) absent; (1) present. Although Horovitz and
Sánchez-Villagra (2003: ch. 3) coded Didelphis as polymorphic, I did not see any specimen in the sample with the atlas transverse foramen completely closed. Notable ontogenetic variation was observed in Metachirus: in subadults and young specimens, the foramen is not completely closed, but shows a small ossified process that tends to close the foramina. This foramen is present in Caluromysiops, Caluromys, Marmosa murina, and M. rubra, but it is absent in most of the didelphid taxa included here. In the sample, individual variation is observed in Philander frenatus, Metachirus, Thylamys pusillus, Marmosa robinsoni, Marmosops noctivagus, and Gracilinanus agilis (coded $\{01\}$ ).

Character 3: Posterior extension of the transverse processes: (0) absent; (1) present. In the same way as Horovitz and SánchezVillagra (2003: ch. 4), I coded this character as present when the transverse processes extend caudally beyond the caudal facets for the axis articulation. Although Horovitz and Sánchez-Villagra (2003) coded Didelphis as polymorphic, in almost the complete sample analyzed here, Didelphis was scored as present. Only in young and subadult specimens is the extension absent, and one specimen of D. virginiana (AMNH 70082) exhibits the transverse process extended to the same level or almost posterior to the caudal facets for the axis. In general, Philander opossum exhibits the condition 0 , but some specimens (e.g., AMNH 190446, 210408) show the processes barely extended beyond the caudal facets (coded $\{01\}$ ).

Character 4: Cranial facets shape: (0) only concave; (1) dorsal edge curved. Although the position of the occipital condyle shows variation in didelphids (Argot, 2003a), I did not note discrete interespecific variation of the atlas cranial facet shape. Here, this character is modified from Horovitz and Sánchez-Villagra (2003: ch. 5), because there are no taxa in the sample with the dorsal edges enveloping the occipital condyle, as shown in some Australasian groups. The dorsal border curved is the most common condition present in the didelphid crown group, although in Thylamys pallidior, Tlacuatzin, and Hyladelphys the cranial facet is only concave, and the polymorphic condition is evidenced in Thylamys venustus. 
Character 5: Development of ventral tubercle: (0) without tubercle; (1) tubercle as a small protuberance, almost absent; (2) tubercle developed; (3) tubercle well developed, laminar shape. The ventral tubercle is a process on the ventral region of the vertebral body. Some muscles implied in neck flexion (such as $\mathrm{m}$. longus colli and $\mathrm{m}$. longus capitis) insert in this structure (Argot, 2003a; see Evans, 1993: fig. 6-31). In didelphids, this tubercle has several degrees of development. For instance, in Philander (state 2) this structure appears more developed than in Didelphis (state 1), whereas in Gracilinanus, Metachirus, Chironectes, and Marmosa rubra the tubercle is well developed and triangular in shape (state 3). In Glironia, Caluromys, Caluromysiops, Lutreolina, Tlacuatzin, and most species of Marmosa the tubercle is present but small. No information is currently available about this character for Thylamys macrurus (coded “?”'). Since I observed intermediate conditions on the development of this structure, the character is treated as ordered $(0 \leftrightarrow 1 \leftrightarrow 2 \leftrightarrow 3)$ in all analyses.

Character 6: Dorsal portion of the caudal articular fovea: (0) caudal articular fovea not posteriorly extended; (1) caudal articular fovea posteriorly extended. The caudal articular fovea appears caudally extended in most of the didelphid species considered here, although it is absent in Didelphis and Caluromysiops, where the fovea is short and not posteriorly extended.

Character 7: Caudal articular fovea shape: (0) caudal fovea oval in shape; (1) caudal fovea round in shape. The caudal articular fovea consists of two cavities that form a freely movable articulation with the second vertebra (Evans, 1993), and it provides for most cranial mobility (Argot, 2003a, 2003b). For this reason, the shape of the fovea has deep implications on head movements. The overall outline is round in shape in several taxa, such as Glironia, Caluromysiops, Caluromys, Philander, Chironectes, Monodelphis, Gracilinanus, Micoureus, Cryptonanus unduaviensis, Marmosa robinsoni, M. murina, Lestodelphys, and Thylamys pusillus. In contrast, it is oval in shape in Didelphis, Metachirus, Marmosops, Marmosa rubra, Thylamys pallidior, and $T$. venustus.
Character 8: Transverse process craniocaudal length with respect to the hemal arches craniocaudal length: (0) longer; (1) equal; (2) shorter. In this character, I compared the craniocaudal development of the transverse process in relation to the dorsal portion of hemal arches, independent of the transverse process posterior extension described in character 3. Several important muscles implied in head movements (e.g., Mm. obliquus capitis caudalis, obliquus capitis cranialis, splenius, and rectus capitis dorsalis minor) are related to the transverse process and the dorsal portion of the hemal arch (Coues, 1869; Filan, 1990; Evans, 1993; Argot, 2003a). Variations in development of hemal arches in some didelphids were evidenced by Argot (2003a: figs. 1, 4). In Didelphis and Philander the transverse process is notably larger than the craniocaudal dimension of the hemal arches, whereas in Chironectes and Lestodelphys there is similar development of both structures. Most of the remaining taxa included here exhibit a small transverse process with respect to the hemal arches. In this pattern, Metachirus exhibits the hemal arches very developed craniocaudally in relation to the transverse process (Argot, 2003a: fig. 1b). Since I observed an intermediate condition in the craniocaudal length of the transverse process with respect to the craniocaudal length of the hemal arches, this character is treated as ordered $(0 \leftrightarrow 1 \leftrightarrow 2 \leftrightarrow 3)$ in all analyses.

Character 9: Lateral extension of the transverse process with respect to the lateral extension of the cranial articular fovea: (0) extended laterally beyond the cranial articular fovea; (1) extended laterally to the same level of the cranial articular fovea. The general pattern in didelphids is the condition 0 . Thus, in most groups considered in this report, the transverse process is more laterally expanded with respect to the cranial articular fovea. To the contrary, a not very laterally expanded transverse process is present in some mouse opossums such as Marmosops pinheiroi Thylamys (except $T$. macrurus), and Gracilinanus.

\section{AXIS}

Character 10: Posterior spinous process extension: (0) absent; (1) present (fig 1). 


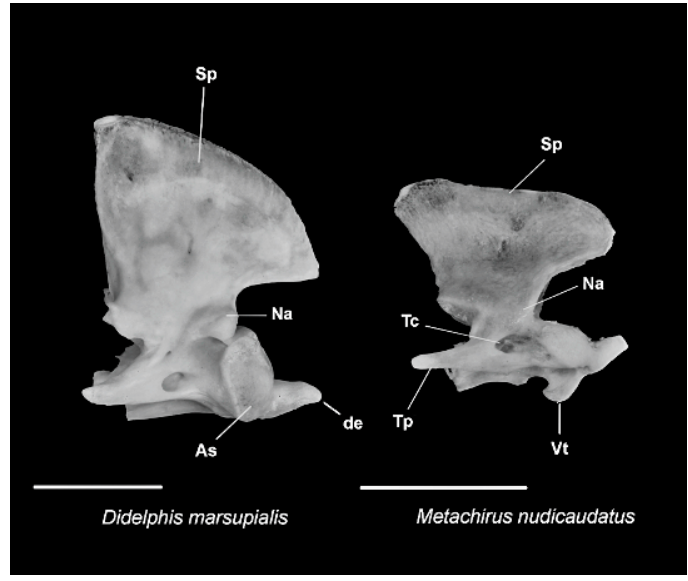

Fig. 1. Didelphis marsupialis (AMNH 21439) and Metachirus nudicaudatus (AMNH 244617), axis in lateral view. The spinous process $(\mathrm{Sp})$ in Metachirus is posteriorly extended (ch. 10[1]), whereas in Didelphis it is not extended beyond the neural arches (Na) (ch. 10[0]). In Didelphis, the anterior extension of the dens (de) is barely anterior to the anterior tip of the spinous process (ch. 11[0]), whereas in Metachirus the dens is cranialy extended (ch. 11[2]). The ventral tubercle (Vt) is a uniform crest in Didelphis (ch. 14[0]), whereas in Metachirus it forms two protruding lobes (ch. 14[1]). Other abbreviations: As, articular surface; Tc, transverse canal; Tp, transverse process. Scale bars: $10 \mathrm{~mm}$.

Similar to Horovitz and Sánchez-Villagra (2003: ch. 10), I coded this character as present when the neural spine extends posteriorly beyond the vertebral arches. Important muscles implied in the head movements, such as $\mathrm{Mm}$. rectus capitis posterior, obliquus capitis caudalis, and spinalis capitis, originate in the axis spinous process (Coues, 1869; Filan, 1990; Evans, 1993; Sargis, 2001; Muizon and Argot, 2003; Argot, 2004a, 2004b). According to Muizon and Argot (2003) and Muizon (1998), the development of the occipital crest in the skull and the craniocaudal extension of the axis spinous process are indicative of a strong musculature of the neck, which is related to predatory habits. In didelphids, the axis spinous process does not show significant variation, since the process is posteriorly extended beyond the neural arches in most taxa included here, except for Didelphis and Hyladelphys, where the posterior extension of the spinous process is absent.
Character 11: Dens cranial extension relative to the cranial tip of the spinous process: (0) to the same level or barely anterior; (1) notably anterior (fig. 1). In head movements, where the atlantoaxial articulation is involved, the dens is used as a pivot (Argot, 2003a). On the other hand, a notable cranial extension of the axis spinous process would restrict neck mobility by contact with the caudal tip of the atlas (Evans, 1993; Sargis, 2001). Some large opossums such as Caluromysiops, Caluromys, Didelphis, and Philander exhibit the dens to the same level or barely anterior with respect to the anterior tip of the spinous process. Most of the remaining taxa show condition 1 , and Tlacuatzin is scored as polymorphic for this character (coded $\{01\}$ ). No information is currently available about this character for Marmosa murina and Gracilinanus microtarsus (coded “?").

Character 12: Axis caudal articular surface fovea shape: (0) rounded shape; (1) oval shape. The caudal articular surface shape is rounded in some taxa, such as Caluromysiops, Philander, Metachirus, Chironectes, Thylamys pallidior, T. venustus, Micoureus regina, Marmosa robinsoni, Marmosops, and Lestodelphys. Instead, an oval caudal articular surface is observed in Glironia, Caluromys, Didelphis, Lutreolina, Thylamys pusillus, Monodelphis, Marmosa rubra, Micoureus demerarae, Tlacuatzin, and Gracilinanus agilis. No information is currently available about this character for Marmosa murina, Gracilinanus microtarsus, and Hyladelphys (coded “?").

Character 13: Inferior portion of neural arches shape in lateral view: (0) narrow; (1) wide (fig. 1). The contact of the neural arches with the vertebral body exhibits two different morphological patterns on the axis. On the one side, a very thin inferior portion of neural arches is observed in several taxa, such as Glironia, Chironectes, Thylamys, Marmosa, Monodelphis, Marmosops, Gracilinanus, and Lestodelphys. In contrast, in Caluromysiops, Caluromys, Didelphis, Lutreolina, Philander, and Metachirus, the inferior portion of neural arches is notably craniocaudally wide. Polymorphism is observed only in Marmosa robinsoni, which usually shows the condition 1, but some specimens (e.g., 
AMNH 257209, 206597, 140332) show the condition 0 (coded $\{01\})$.

Character 14: Ventral tubercle shape: (0) uniform crest; (1) two separate lobes; (2) without crest or lobe (fig. 1). The ventral tubercle of the axis exhibits different patterns in the taxa analyzed here. In some of them, such as Caluromysiops, Caluromys, Didelphis, Philander, Chironectes, Micoureus, Marmosa murina, and Gracilinanus agilis the ventral tubercle is simply an anteroposteriorly elongated uniform crest. A more complex shape is observed in Glironia, Metachirus, Lutreolina, Thylamys, Monodelphis, some species of Marmosa, Marmosops, and Lestodelphys, where the ventral tubercle takes the form of two separate lobes anteroposteriorly aligned. Finally, no trace of a crest or lobe is observed in Tlacuatzin. No information is currently available about this character for Gracilinanus microtarsus (coded "'?"). As none of the conditions of the ventral tuberlcle morphology appears to be intermediate to the others, I treated this character as unordered.

Character 15: Neural arch cranial notch shape: (0) narrow; (1) wide. The notch in the cranial border of neural arch exhibits different morphologies in the taxa analyzed here. Caluromys, Philander, Didelphis, Lutreolina, Micoureus demerarae, Marmosops impavidus, and $M$. noctivagus show the notch narrow. In other groups, such as Glironia, Chironectes, Metachirus, Thylamys, Tlacuatzin, Monodelphis, Micoureus regina, Marmosa, Gracilinanus, Marmosops parvidens, M. pinheiroi, Cryptonanus, and Lestodelphys, the notch is notably wide. In the limited postcranial sample of Caluromysiops (see appendix 1), I observed polymorphism in this character, because in one specimen (AMNH 208101) the notch is wide, while in the other (AMNH 244364) the notch is markedly narrow (coded $\{01\})$.

\section{Posterior Cervical Vertebrae}

Character 16: C5 and T1 craniocaudal body length: (0) C5 and T1 craniocaudal body length subequal; (1) C5 craniocaudal body length shorter than T1. This character was described by Horovitz and SánchezVillagra (2003: ch. 18). The C5 and T1 body length determines the gap between vertebrae in the cervico-thoracic junction, a functionally important area where changes in the skeletal configuration occur during locomotion (Herbin et al., 2000). Most didelphids have the C5 craniocaudal body length subequal to or longer than T1. Marmosa rubra is autapomorphic in this character, because it shows the $\mathrm{C} 5$ body shorter than the $\mathrm{T} 1$ body.

Character 17: C6 spinous process shape: (0) absent; (1) protuberance; (2) lamina (fig. 4). The shape and development of the spinous process in posterior cervical vertebrae are important because it is where part of the deep musculature of the neck attaches, such as Mm. spinalis cervicis and multifidus cervicis (Mann Fischer, 1956; Argot, 2003a, 2003b; Evans, 1993). The shape variations of the C6 spinous process were described by Horovitz and Sánchez-Villagra (2003: ch. 20). In didelphids, different conditions in the C6 spinous process are present. Caluromys, Caluromysiops, Didelphis, Philander frenatus, P. mcilhennyi, Metachirus, Lutreolina, Marmosa robinsoni, M. murina, Marmosops fuscatus, and Lestodelphys show the C6 spinous process as laminar. Only a small protuberance is observed in Glironia, Philander opossum, Mamrosa rubra, Thylamys pallidior, T. macrurus, Monodelphis, Micoureus demerarae, Marmosa mexicana, and Marmosops (except M. noctivagus). Lastly, the spinous process in C6 is completely absent in Tlacuatzin, Thylamys pusillus, T. venustus, Cryptonanus unduaviensis, and Gracilinanus microtarsus. Additionally, polymorphism is observed in Micoureus regina, Gracilinanus agilis, and Marmosops noctivagus, as in some specimens the process is absent, whereas in others there is a small protuberance (coded $\{01\})$. As I observed an intermediate condition in the C6 spinous process shape, this character is treated as ordered $(0 \leftrightarrow 1 \leftrightarrow 2)$ in all analyses.

Character 18: C7 transverse foramen: (0) absent; (1) present. This character is modified from Horovitz and Sánchez-Villagra (2003: ch. 21), since here I did not observe any taxa with the $\mathrm{C} 7$ transverse foramen incipient. The C7 transverse foramen is present in Glironia, Caluromysiops, Caluromys, Didelphis, Philander opossum, P. frenatus, Chironectes, Marmosa rubra, and Monodelphis adusta. This foramen is absent in Philander mcilhennyi, 


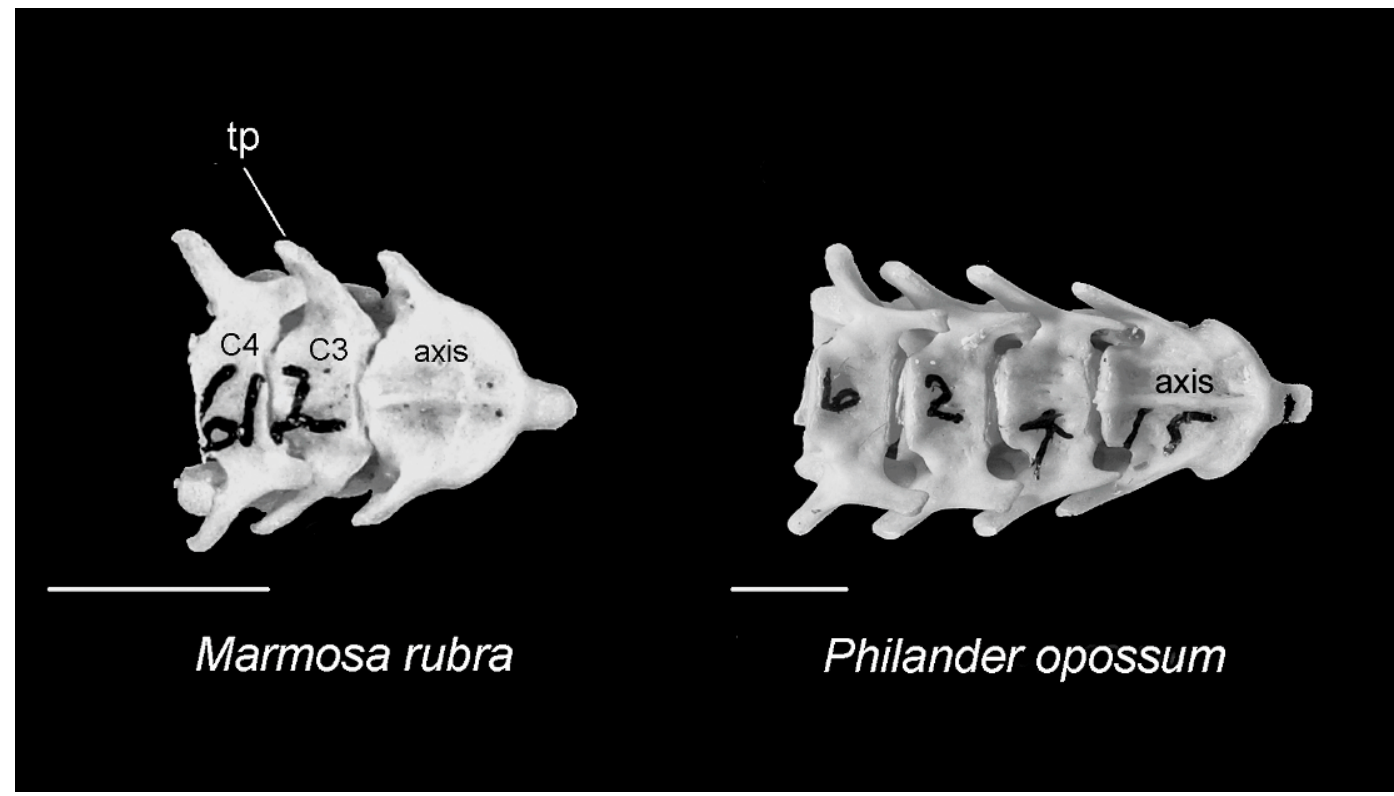

Fig. 2. Marmosa rubra (FMNH 124612) and Philander opossum (AMNH 262415), anterior cervical vertebrae in ventral view. In Marmosa rubra, the transverse process (Tp) on the third cervical (C3) has one head (ch. 21[0]), but in Philander it shows an anterior head (h) (ch. 21[1]). In both species the fourth cervical (C4) has two heads (ch. 22[1]). Scale bars: $5 \mathrm{~mm}$.

Lutreolina, Tlacuatzin, Thylamys, Monodelphis brevicaudata, M. theresa, Marmosa robinsoni, M. mexicana, M. murina, Marmosops, Gracilinanus, Cryptonanus, and Lestodelphys. The polymorphic condition is present in Metachirus, Micoureus demerarae, and $M$. regina (coded $\{01\})$.

Character 19: C3-C7 spinous process size with respect to the spinous process of the axis: (0) lesser; (1) subequal (figs. 3, 4, 6). In general, didelphids show the spinous process of posterior cervical vertebrae shorter than that of the axis. Condition 1 is exhibited only in Didelphis, which has a rigid neck related with the hypertrophy of the spinous processes, and the particularly strong articulation in cervical vertebrae. This special morphology prevents the dorsiflexion and lateral movements of the neck, but it is difficult to relate it to any diet or locomotor specialization in Didelphis (Argot, 2003a).

Character 20: Relative size between C6 and C7 spinous process: (0) C7 spinous process taller than C6 spinous process; (1) $\mathrm{C} 6$ and $\mathrm{C} 7$ spinous process of similar size (fig. 4). I observed the C7 spinous process to be taller than C6 spinous process in Glironia,
Caluromysiops, Caluromys, Philander, Lutreolina, Tlacuatzin, Thylamys pusillus, Marmosa robinsoni, M. rubra, Marmosops parvidens, Cryptonanus unduaviensis, Gracilinanus agilis, and Lestodelphys. In contrast, C6 and C7 spinous processes have a similar size in Didelphis, Metachirus, Chironectes, Thylamys pallidior, T. macrurus, Monodelphis, Micoureus, Marmosa mexicana, M. murina, Marmosops (except M. parvidens), and Gracilinanus microtarsus. In the sample of Thylamys venustus, I found a specimen (AMNH 261245) with a small C7 spinous process and another (AMNH 261253) without this process. Similarly, Micoureus regina exhibits the polymorphic condition, as a male specimen has the $\mathrm{C} 7$ spinous process taller than the C6 spinous process, whereas in a female specimen the spinous processes of both vertebrae are similar in size (coded $\{01\}$ ). No information is currently available about this character for Marmosops pinheiroi (coded “?").

Character 21: C3 transverse process heads: (0) one head; (1) two heads (fig. 2). The anterior part of the transverse process (called inferior lamellae) is present in some cervical 
vertebrae in didelphids. Some essential muscles related with neck flexion, such as $\mathrm{Mm}$. longus colli (pars cervicalis), longissimus cervis, intertransversarii dorsales cervicis, and intertransversarii ventrales cervicis (see Evans, 1993: figs. 6-28, 6-30; Argot, 2003a: fig. 4a), originate in this structure. In C3, the anterior portion of the transverse process is present in several taxa, such as Caluromysiops, Caluromys, Didelphis, Philander, Chironectes, Metachirus, and Lutreolina. On the other hand, in Glironia and mouse opossums this structure is absent.

Character 22: $\mathrm{C} 4$ transverse process heads: (0) one head; (1) two heads (fig. 2). The lamellae in the $\mathrm{C} 4$ transverse process are present in most species treated here. However, in some small opossums, such as Thylamys pusillus, T. macrurus, Monodelphis adusta, M. theresa, Lestodelphys, and Hyladelphys, the lamellae on $\mathrm{C} 4$ are absent. Individual variation in this character is common in Thylamys pallidior, Marmosops impavidus, and Thylamys venustus, Marmosa robinsoni, and Cryptonanus unduaviensis (coded $\{01\})$.

Character 23: C7 transverse process direction: (0) lateral; (1) ventrolateral. According to Argot (2003a), the C7 transverse process is anteriorly oriented in some taxa such as Metachirus. However, the variation of this process in didelphids is focused here on a lateral or ventrolateral $\mathrm{C} 7$ transverse process orientation. In some taxa, such as Glironia, Caluromys, Caluromysiops, Didelphis, Thylamys pallidior, T. macrurus, Marmosa rubra, and $M$. mexicana, the process is ventrolateral. On the other hand, in Metachirus, Philander, Lutreolina, Chironectes, Tlacuatzin, Thylamys pusillus, T. venustus, Monodelphis, Micoureus, Lestodelphys, Marmosa murina, Marmosops, Gracilinanus, Cryptonanus, and Hyladelphys, the process is laterally oriented.

\section{ThORACiC Vertebrae}

Character 24: First thoracic vertebra with a tall spinous process relative to other vertebrae: (0) T1; (1) T2 (fig. 3). The muscular system that is located in the first two thoracic vertebrae is complex (MannFischer, 1956; Filan, 1990). The M. splenius

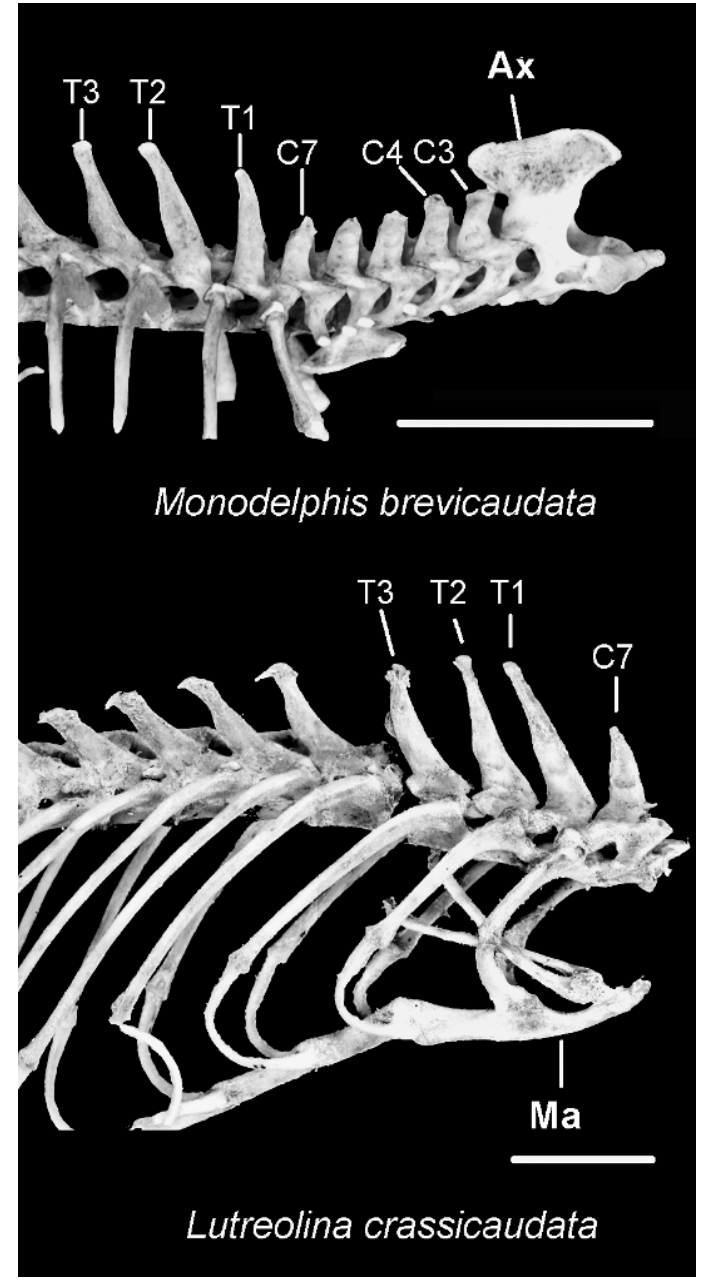

Fig. 3. Monodelphis brevicaudata (AMNH 257203) and Lutreolina crassicaudata (AMNH 133250), partial vertebral series in lateral view. In Monodelphis, C3-C5 spinous processes are lower than the axis spinous process (ch. 19[0]). The first thoracic vertebra with tall spinous process relative to other vertebrae is placed in the $\mathrm{T} 2$ position in Monodelphis (ch. 24[1]), but it is in the T1 position in Lutreolina (ch 24[0]). Abbreviation: Ma, manubrium of sternum. Scale bars: $10 \mathrm{~mm}$.

(which is involved in the support and movements of the head) originates in the spinous process of T1 (Argot, 2003a: fig. 4a; Evans, 1993). This character is modified from Horovitz and Sánchez-Villagra (2003: ch. 26), because it appears as multistate, with a third condition indicating the spinous process tall in T3. However, according to their published 


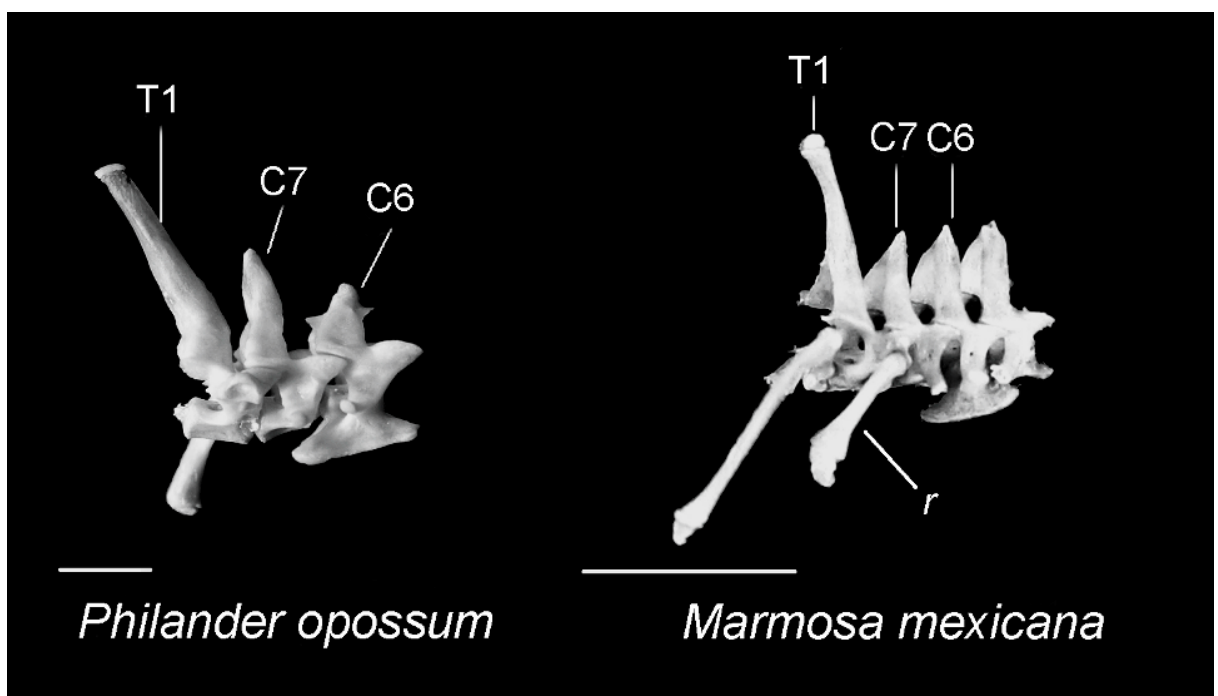

Fig. 4. Philander opossum (AMNH 262415) and Marmosa mexicana (ROM 99608), last cervical vertebrae and first thoracic vertebra in lateral view. In Philander, the C7 spinous process is taller than the C6 process (ch. 20[0]), whereas in Marmosa mexicana the C6 and C7 spinous processes are similar in size (ch. 20[2]). Note the C6 spinous process resembling a protuberance in both species (ch. 17[2]). Abbreviation: r, rib. Scale bars: $5 \mathrm{~mm}$.

data matrix, there are not terminals with this condition. In the same work, the authors reported polymorphism in Didelphis virigini$a n a$, although in the sample examined, I did not find evidence of any individual variation in this species. All didelphid groups show spinous process of $\mathrm{T} 1$ tall relative to other vertebrae, except for Monodelphis brevicaudata and $M$. theresa, where the first tall spinous process is placed in $\mathrm{T} 2$.

Character 25: First thoracic vertebra with prezygapophysis facing laterally: (0) T2; (1) T3. This character was defined by Horovitz and Sánchez-Villagra (2003: ch. 27). All didelphid groups display T3 with prezygapophysis facing laterally, except for Caluromys and Caluromysiops, where the first prezygapophysis facing laterally is in T2. This condition is common in some Autralidelphians, such as Dromiciops, diprotodontians, and the dasyurid Myoictis (Horovitz and Sánchez-Villagra, 2003).

Character 26: T1 body craniocaudally extended in dorsal view: (0) absent; (1) present (fig. 5). The vertebral body extended craniocaudally in T1 occurs only in Caluromysiops and Didelphis.
Character 27: Position of the first thoracic vertebra with a low and craniocaudally expanded spinous process: (0) T10; (1) T11; (2) T12; (3) T13. The spinous process is low and craniocaudally enlarged in the posterior thoracic vertebrae, casually coincident with the position of the diaphragmatic vertebra. A short and wide spinous process restricts spinal mobility by decreasing intervertebral space (Sargis, 2001). In most taxa included in this analysis, the location of this kind of spinous process starts on T10. However, some species show variations departing from the most common condition. In Glironia, Philander frenatus, Marmosa robinsoni, Marmosops noctivagus, $M$. incanus, $M$. parvidens, M. pinheiroi, Cryptonanus unduaviensis, and Gracilinanus microtarsus, the process starts on T11; in Lestodelphys and Tlacuatzin, it starts on T12, whereas in Chironectes and Hyladelphys it starts on T13. The posterior position of this kind of spinous process in Chironectes indicates freer movements of the vertebral spine on the anterior portion, perhaps related to the particular swimming characteristic of this species. However, the specific mode of locomotion in Hyladelphys 


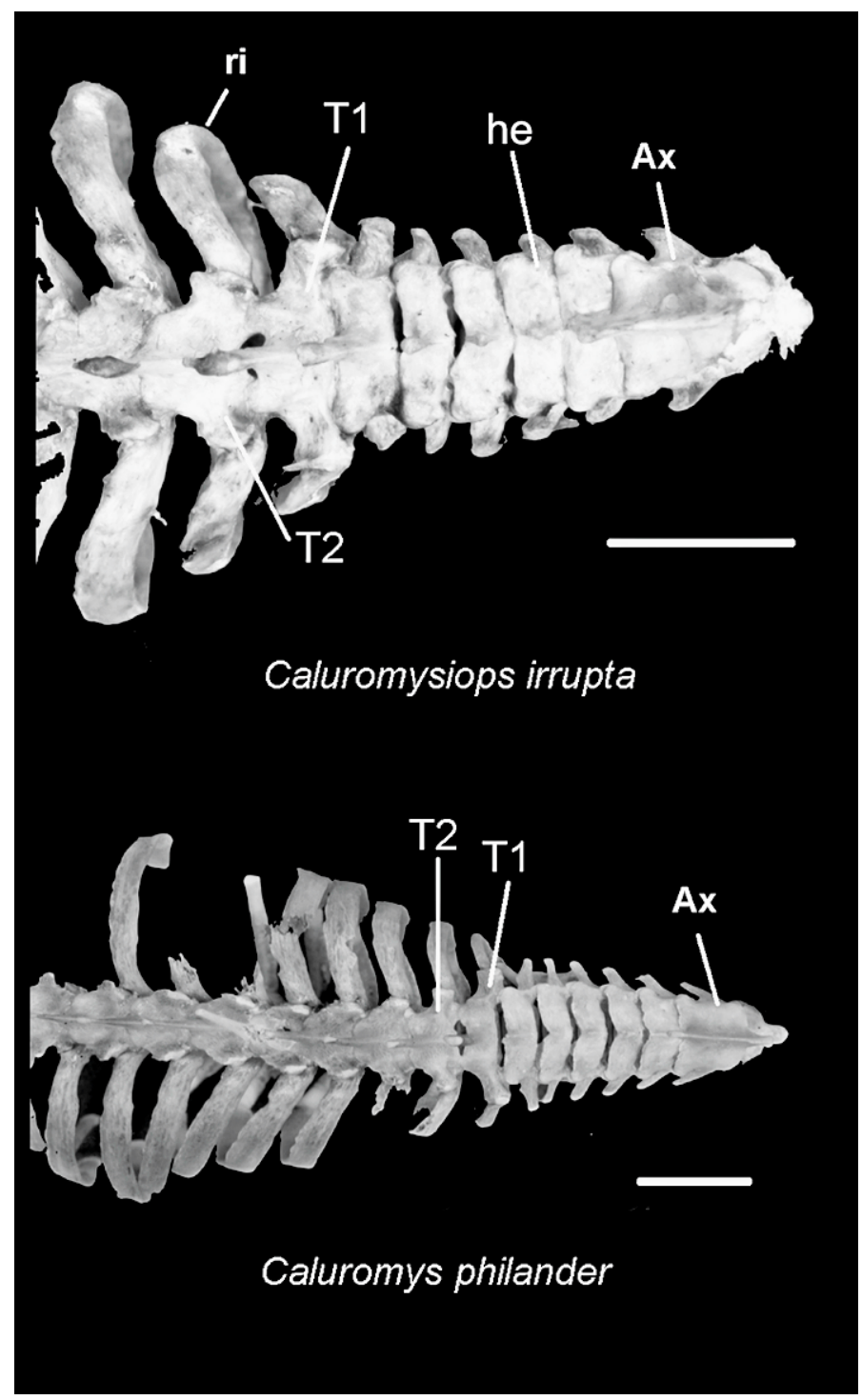

Fig. 5. Caluromysiops irrupta (AMNH 208101) and Caluromys philander (AMNH 267001), cervical and thoracic vertebrae in dorsal view. The T1 body in Caluromysiops is lengthened craniocaudally (ch. 26[1]), whereas this condition is not observed in Caluromys (ch. 26[0]). However, in both taxa the hemal arches in $\mathrm{T} 1$ are notably shorter than hemal arches in T2 (ch. 32[1]). Note the ribs (ri) flattened and craniocaudally expanded in both taxa (ch. 49[0]). Other abbreviations: Ax, axis; he, hemal arch. Scale bars: $10 \mathrm{~mm}$.

is unknown. Due to the fact that Lutreolina does not show this kind of vetebra, I scored it as inapplicable ("-") for this character. Because I observed intermediate conditions in the position of the first thoracic vertebra with a low and craniocaudally expanded spinous process, this character is treated as ordered $(0 \leftrightarrow 1 \leftrightarrow 2 \leftrightarrow 3)$ in all analyses.
Character 28: Position of the diaphragmatic vertebra: (0) T8; (1) T10; (2) T11; (3) T12; (4) T13. The mammillary process in the region is more developed in the posterior vertebrae. The diaphragmatic vertebrae represent a point of transition in the vertebral column, where the articulation displays a different articular mode, and therefore it 
becomes functionally distinct (Argot, 2003a). Posterior to the diaphragmatic vertebra, the thoracic vertebrae become morphologically lumbarlike (Argot, 2003a). In this point, the contact between facets is more obliquely oriented, and therefore it reduces lateral bending and log-axis rotation of the vertebral column, allowing a sagittal flexion-extension of the spine (Rockwell et al., 1938; Washburn and Buettner-Janush, 1952; Pridmore, 1992; Shapiro and Jungers, 1994). The mammillary process of the diaphragmatic and postdiaphragmatic vertebrae is the point of origin of tendons for M. longissimus dorsi, a powerful extensor of the back (Argot, 2003a), and M. multifidus thoracis, which (along with other dorsal back muscles) fixes the vertebral column, especially in bilateral action (see Evans, 1993: fig. 6-30). Thus, the position of the diaphragmatic vertebra has an important functional significance.

I found a high variation in the location of the diaphragmatic vertebra among didelphids. In Caluromys lanatus, Caluromysiops, Philander opossum, and P. frenatus, the position is in T10. In Glironia, Caluromys philander, Metachirus, Philander mcilhennyi, Lestodelphys, Lutreolina, Chironectes, Marmosa rubra, Thylamys pusillus, T. macrurus, Monodelphis brevicaudata, M. adusta, Micoureus demerarae, and Marmosops incanus, the position is in T11. In Tlacuatzin, Thylamys pallidior, $T$. venustus, Monodelphis theresa, Marmosa robinsoni, M. mexicana, M. murina, Marmosops impavidus, M. noctivagus, M. pinheiroi, Cryptonanus unduaviensis, and Gracilinanus, this vertebra is in T12. Only Micoureus regina shows this vertebra in T13, and in Didelphis it is placed in T8, although according to Argot (2003a), this element is placed in the T9 position. Because I observed intermediate conditions in the position of the diaphragmatic vertebra, this character is treated as ordered $(0 \leftrightarrow 1 \leftrightarrow 2 \leftrightarrow$ $3 \leftrightarrow 4$ ) in all analyses.

Character 29: Position of the anticlinal vertebra: (0) T10; (1) T11; (2) L4; (3) L5; (4) L6; (5) S1. According to most previous definitions, the anticlinal vertebra is the element in which the orientation of the spinous process reverses from posterior to anterior (Mann Fischer, 1953; Evans, 1993; Shapiro, 1995; Muizon and Argot, 2003;
Kurz, 2005). This morphology is a consequence of the tractions exerted by the common epaxial musculature, whose fibers insert on the tip of these processes (Mann Fischer, 1953; Muizon and Argot, 2003). This pattern is part of a functional complex that points toward the sagittal flexibility required for quadrupedal locomotion (Rockwell et al., 1938; Howell, 1965; Shapiro, 1995). According to Argot (2003a), there is no anticlinal vertebra in Caluromys and Philander, because all spinous process are posteriorly oriented. This is a general pattern evidenced in most didelphids (except for Metachirus and some species of Marmosops). However, and following the criterion of Evans (1993), I consider the anticlinal vertebra that in which the spinous process is nearest perpendicular to the long axis of the vertebral body, independent of the orientation of the spinous process on the posterior vertebrae. In this sense, an anticlinal vertebra is exhibited in didelphids, but its position is highly variable.

In some taxa, the anticlinal vertebra is placed posteriorly in the lumbar region, as in Caluromys and Monodelphis theresa, where it is observed on L6; or Philander, Tlacuatzin, Thylamys pusillus, T. macrurus, Marmosops pinheiroi, Micoureus, and Cryptonanus, where the anticlinal vertebra is on L5. The anticlinal vertebra is in L4 in Hyladelphys, Lestodelphys, Marmosops parvidens, Thylamys venustus, and T. pallidior. Other groups, such as Didelphis, Metachirus, Monodelphis adusta, Marmosa robinsoni, M. mexicana, Marmosops incanus, and Gracilinanus microtarsus, have the anticlinal vertebra in T11. The last group, which is composed of Chironectes, Monodelphis brevicaudata, Marmosa murina, Marmosops imavidus, and $M$. noctivagus, has the anticlinal vertebra located in T10. According to Argot (2003a), this element is in the third lumbar vertebra in Metachirus, but I observed that vertebra in the T11 position. As I observed intermediate conditions in the position of the anticlinal vertebra, this character is treated as ordered $(0 \leftrightarrow 1 \leftrightarrow 2$ $\leftrightarrow 3 \leftrightarrow 4 \leftrightarrow 5$ ) in all analyses.

Character 30: Position of the first vertebra where the accessory process is differentiated from the transverse process: (0) T6; (1) T7; (2) T8; (3) T9. The accessory process (called 
anapophysis by some authors) appears in the posterior thoracic vertebrae. The accessory processes protrude posteriorly and lock the articulation with the following vertebra, restricting lateral flexibility. The transverse and accessory processes are completely separated in different vertebral positions. In Caluromys and Philander mcilhennyi the position is in T9, whereas in Didelphis, Philander opossum, P. frenatus, Metachirus, Lutreolina, Monodelphis theresa, Micoureus regina, Marmosa robinsoni, M. mexicana, Marmosops impavidus, M. noctivagus, Cryptonanus unduaviensis, and Gracilinanus agilis, the position is in T8. A third condition includes Caluromysiops, Chironectes, Tlacuatzin, Thylamys, Monodelphis brevicaudata, $M$. adusta, Micoureus demerarae, Marmosa murina, $M$. rubra, Marmosops incanus, $M$. parvidens, $M$. pinheiroi, and Gracilinanus microtarsus, where the position is in T7. Lastly, only Lestodelphys and Glironia show this vertebra in the T6 position. As I observed intermediate conditions in the position of the first vertebra where the accessory process is differentiated from the transverse process, this character is treated as ordered $(0 \leftrightarrow 1 \leftrightarrow 2 \leftrightarrow 3)$ in all analyses.

Character 31: Caudal enlargement of the postzygapophysis in thoracic vertebrae 2-8: (0) absent; (1) present. In dorsal view, the postzygapophysis of the thoracic vertebrae 28 appears caudally enlarged only in Glironia and in the majority of small opossums (Tlacuatzin, Marmosa, Micoureus, Marmosops, Thylamys, Monodelphis, Gracilinanus, Cryptonanus, and Lestodelphys).

Character 32: Hemal arches in T1 notably shorter (craniocaudally) than hemal arches in T2: (0) absent; (1) present (fig. 5). Most of the taxa treated exhibit condition 0 , except for Glironia, Caluromys, and Caluromysiops, in which the hemal arches in $\mathrm{T} 1$ are craniocaudally shorter than the hemal arches in $\mathrm{T} 2$.

Character 33: Spinous process posterior to the anticlinal vertebra cranially oriented: (0) absent; (1) present. As mentioned for character 29 , the anticlinal vertebra is the element in which the orientation of the spinous process is almost perpendicular in relation to the horizontal plane of the body. The most common pattern evidenced in didelphids is

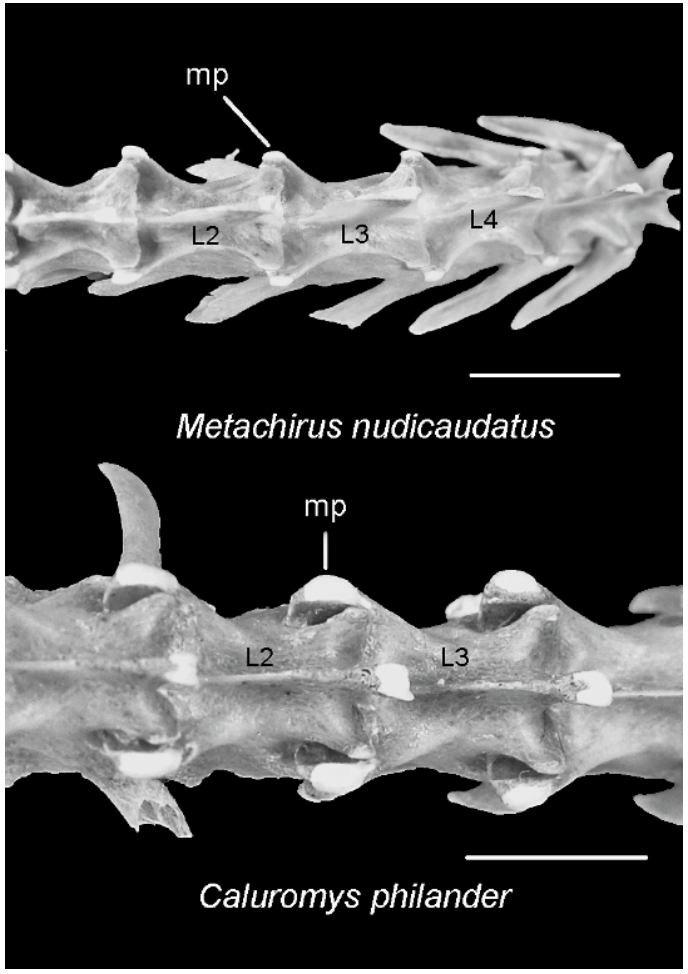

Fig. 6. Metachirus nudicaudatus (AMNH 267009) and Caluromys philander (AMNH 267001), lumbar vertebrae in dorsal view. In Metachirus the mammillary process (mp) of L3 is not anteriorly extended beyond the articulation with L2 (ch. 34[0]), whereas in Caluromys it is extended (ch. 34[1]). Scale bars: $10 \mathrm{~mm}$.

that in which the spinous process of the postanticlinal vertebra does not change its orientation. Only in Metachirus, Marmosops parvidens, and $M$. pinheiroi are the spinous processes in postanticlinal vertebrae anteriorly oriented.

\section{LUMbar VERTEBRAE}

Character 34: Extension of mammillary process in L3: (0) not beyond the anterior vertebra; (1) scarcely beyond the anterior vertebra (fig. 6). This character is modified from Horovitz and Sánchez-Villagra (2003: ch. 30) in which I consider different levels of anterior extension in the mammillary process (called metapophysis in the referenced work). The mammillary process in L3 is barely extended anteriorly in Glironia, Caluromys, 


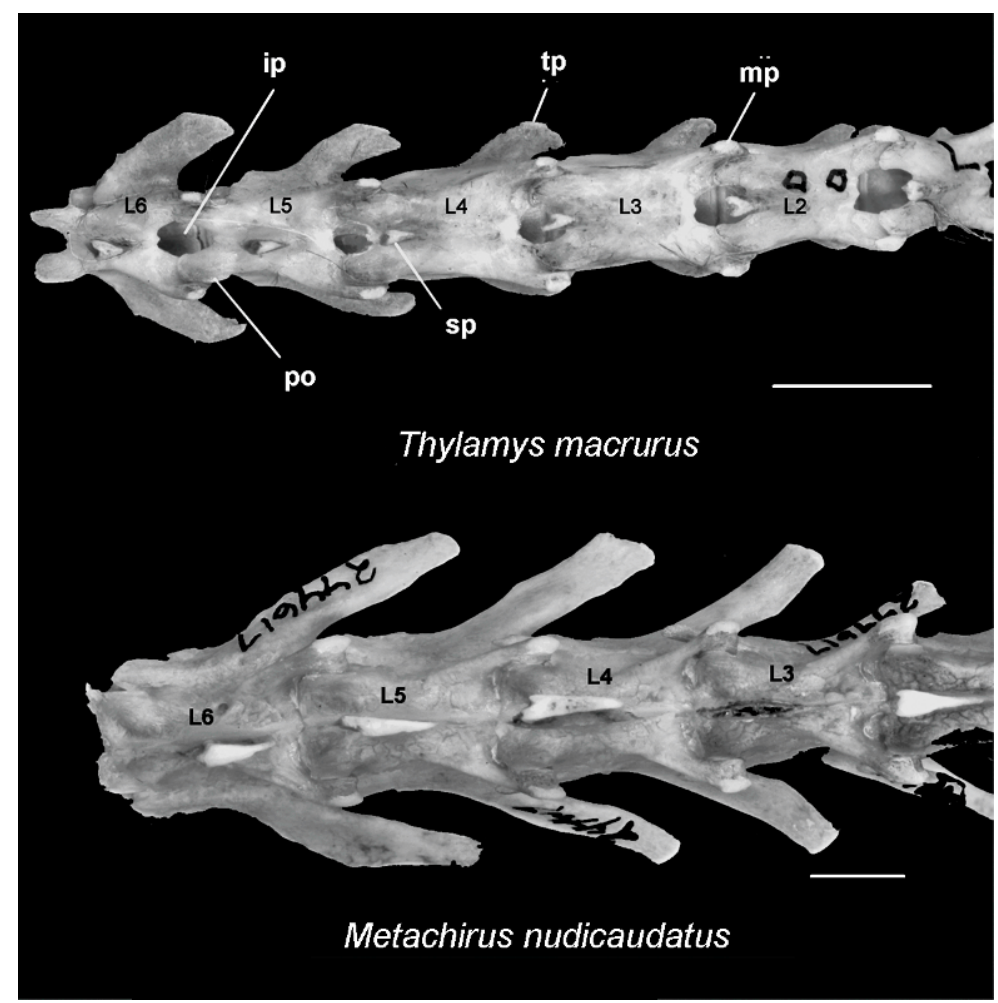

Fig. 7. Thylamys macrurus (MSB 70700) and Metachirus nudicaudatus (AMNH 244617), lumbar vertebrae in dorsal view. An intervertebral space (ip) is evident dorsally in Thylamys (ch. 36[0]), whereas in Metachirus this space is absent (ch. 36[1]). Other abbreviations: mp, mammillary process; po, postzygapophysis; sp, spinous process; tp, transverse process. Scale bar: $5 \mathrm{~mm}$.

Caluromysiops, Chironectes, Micoureus demerarae, Marmosa robinsoni, Marmosops noctivagus, $M$. incanus, and Cryptonanus unduaviensis. Contrary to that, in Metachirus, Lutreolina, Philander, Didelphis, Tlacuatzin, Thylamys, Monodelphis, Marmosa mexicana, M. murina, Marmosops impavidus, M. pinheiroi, M. parvidens, and Gracilinanus the mammillary process does not extend beyond the anterior vertebra. Individual variation is observed just in Micoureus regina (coded $\{01\}$ ).

Character 35: Last lumbar vertebra, foramen on dorsal arch: (0) absent; (1) present. Although this character was described by Horovitz and Sánchez-Villagra (2003: ch. 29) only for the last lumbar vertebra, the foramen is present in all lumbar and most posterior thoracic vertebrae. I had seen evidence of individual variation and asymmetry in some didelphid taxa. Horovitz and
Sánchez-Villagra (2003) coded Didelphis as 0, but I found notable asymmetry and individual variation in the sample analyzed (e.g., $D$. albiventris AMNH 205385; D. marsupialis AMNH 235003, 209164). Similarly, in most specimens of Caluromys lanatus the foramen is present, but in specimen AMNH 133199 it is absent. In the sample analyzed of Chironectes the asymmetry is the general condition. In the same way, some specimens of Marmosa robinsoni having no foramen are observed (AMNH 206596, 257210), and $M$. murina (AMNH 13659), Marmosops impavidus (AMNH 61382), and $M$. noctivagus (AMNH 136157) also exhibit asymmetry (coded $\{01\}$ ). Only Marmosa mexicana lacks the foramina.

Character 36: Intervertebral space in dorsal view: (0) intervertebral space evident; (1) intervertebral space reduced or absent (fig. 7). Glironia and the mouse opossums 


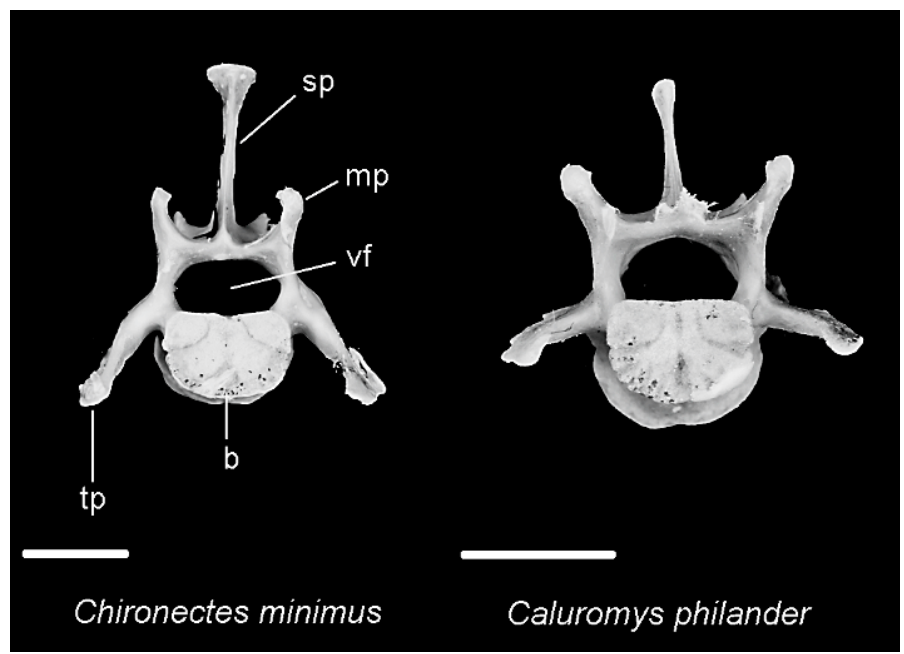

Fig. 8. Chironectes minimus (AMNH 264571) and Caluromys philander (AMNH 267001), fifth lumbar vertebra in anterior view. In Chironectes, the transverse process (tp) is ventrally extended beyond the vertebral body (b) (ch. 37[1]), whereas in Caluromys it is not extended beyond the vertebral body, being more laterally extended (ch. 37[0]). Other abbreviations: mp, mammillary process; sp, spinous process; vf, vertebral foramen. Scale bars: $5 \mathrm{~mm}$.

have an evident intervertebral space in dorsal view, between the hemal arches of two contiguous vertebrae. In large opossums (including Caluromys and Caluromysiops) such space is absent.

Character 37: Ventral extension of L4-L6 transverse process: (0) not extended beyond the vertebral body; (1) extended beyond the vertebral body (fig. 8). The morphology of the lumbar transverse process has been related to the ventral flexion of the column (Shapiro, 1993, 1995; Johnson and Shapiro, 1998). In bounding-running forms, the ventral extension of the transverse process is related to an increased range of sagittal flexion (Shapiro, 1995, Sargis, 2001; Argot, 2003a) and creates a major space for epaxial musculature, particularly the lumbar division of m. longissimus dorsi (Sanders and Bodenbender, 1994; Shapiro, 1995, Sargis, 2001). The transverse process is ventrally extended beyond the vertebral body in some large opossums, except for Glironia, Caluromys, Caluromysiops, Didelphis, Philander opossum, and $P$. mcilhennyi, where the transverse process is not extended ventrally; a similar condition is also observed in mouse opossums. Instead, in Metachirus, Lutreolina, Chironectes, P. frenatus, and Hyladelphys the transverse process protrudes cranially and ventrally.

\section{Sacrum and Caudal Vertebrae}

Character 38: Number of vertebrae in contact with ilium: (0) one vertebra, (1) two vertebrae (fig. 9). This is a conservative character in the context of the taxonomic sample analyzed here, as most taxa analyzed here exhibit two vertebrae fused or in contact with ilium, except for Glironia and Hyladelphys, where only one vertebra ( $\mathrm{S} 1)$ is fused to ilium.

Character 39: Posterior process on the lateral sacral crest on S2: (0) absent; (1) present (figs. 9, 10). In general, didelphid taxa show a posterior process on $\mathrm{S} 2$, except for Glironia, Marmosa rubra, Lestodelphys, and Hyladelphys, where this process is clearly absent. No information is currently available about this character for Gracilinanus microtarsus (coded "'?").

Character 40: Spinous process on sacrum: (0) spinous process just in $\mathrm{S} 1$; (1) spinous process present on S1 and S2 (fig. 11). Most of the didelphid group has a spinous process in both sacral vertebrae, except for Glironia, Marmosops parvidens, and M. incanus, where 


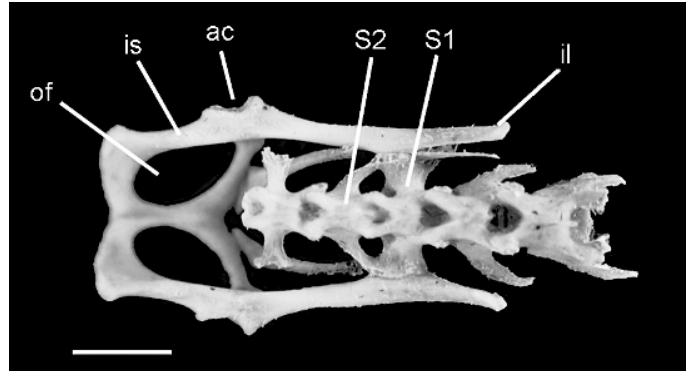

Hyladelphys kalinowskii

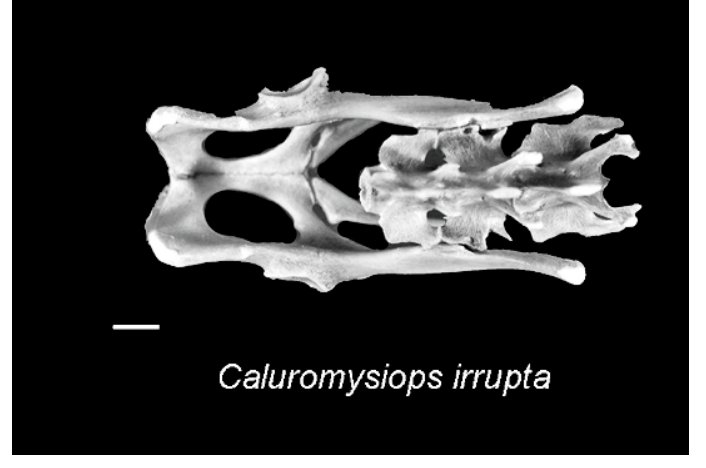

Fig. 9. Hyladelphys kalinowskii (RSV 1572) and Caluromysiops irrupta (AMNH 244364), pelvis, sacrum, and posterior lumbar vertebrae in dorsal view. In Hyladelphys, just the first sacral vertebra (S1) is contacting the illium (il) (ch. 38[0]), whereas in Caluromysiops both sacral elements (S1 and S2) are contacting the illium (ch. 38[1]). Note the absence of the posterior process on the lateral sacral crest of S2 in Hyladelphys (ch. 39[0]). Other abbreviations: ac, acetabulum; is, ischium; of, obturator foramen. Scale bars: $5 \mathrm{~mm}$. the process on S2 is absent. However, there is individual variation in the sample of Thylamys venustus, Marmosops noctivagus, and $M$. pinheiroi (coded $\{01\}$ ). No information is currently available about this character for Gracilinanus microtarsus (coded "?"').

Character 41: Spinous process size on $\mathrm{S} 1-$ S2: (0) S1 and S2 spinous processes of similar size; (1) $\mathrm{S} 1$ spinous process taller than $\mathrm{S} 2$ (fig. 11). In most didelphids, $\mathrm{S} 1$ and $\mathrm{S} 2$ have similar spinous process sizes. Only in some taxa, such as Philander frenatus, P. mcilhennyi, Lutreolina, Chironectes, Metachirus, Marmosops pinheiroi, Thylamys macrurus, and Hyladelphys, the $\mathrm{S} 1$ spinous process is taller than that of S2. The taxa coded 0 in character 40 are treated as inapplicable ("_,") for this character. No information is currently available about this character for Gracilinanus microtarsus (coded "?')).

Character 42: Ventral foramina on S1 body: (0) absent; (1) present (fig. 12). All taxa analyzed exhibit a ventral foramen on S1 body, except Glironia, Caluromys, Caluromysiops, and Hyladelphys.

Character 43: Caudal vertebra with transverse process length similar to vertebral body length: (0) absent; (1) present. In all taxa incuded in the analysis, the transverse process of the anterior caudal vertebrae forms a strong structure that protrudes laterally and expands craniocaudally. The muscles that insert in the apex of these processes are $\mathrm{Mm}$. ischio-caudalis and abductor caudae dorsalis,

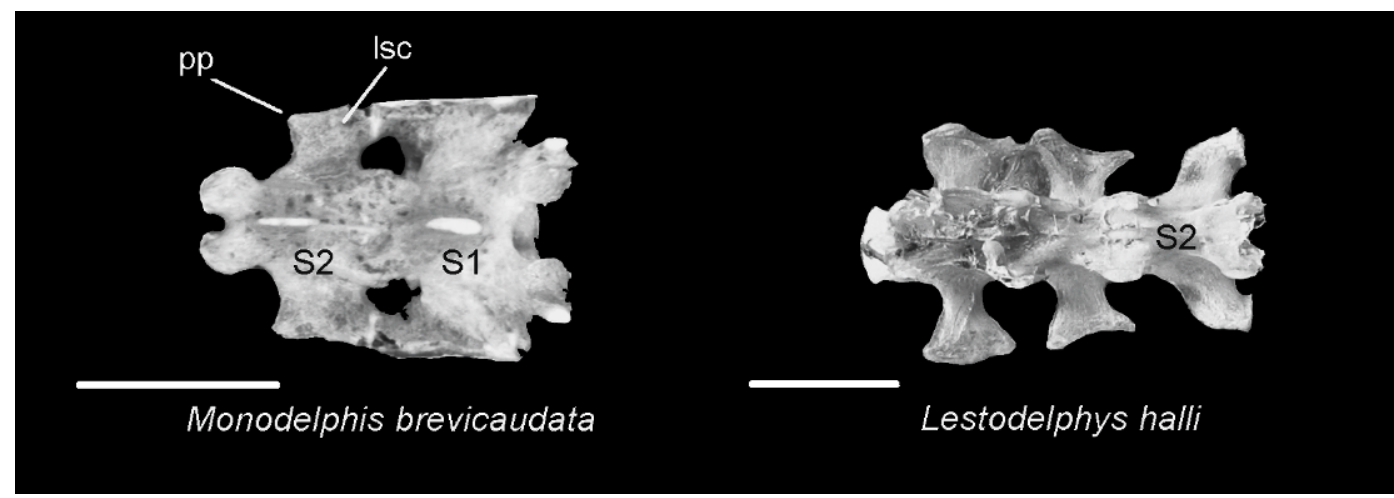

Fig. 10. Monodelphis brevicaudata (AMNH 257203) and Lestodelphys halli (UWZM 22422), sacral vertebrae in dorsal view. In Monodelphis there is a posterior process (pp) on the lateral sacral crest (lsc) on S2 (ch. 39[1]), whereas in Lestodelphys it is absent (ch. 39[0]). Scale bars: $5 \mathrm{~mm}$. 


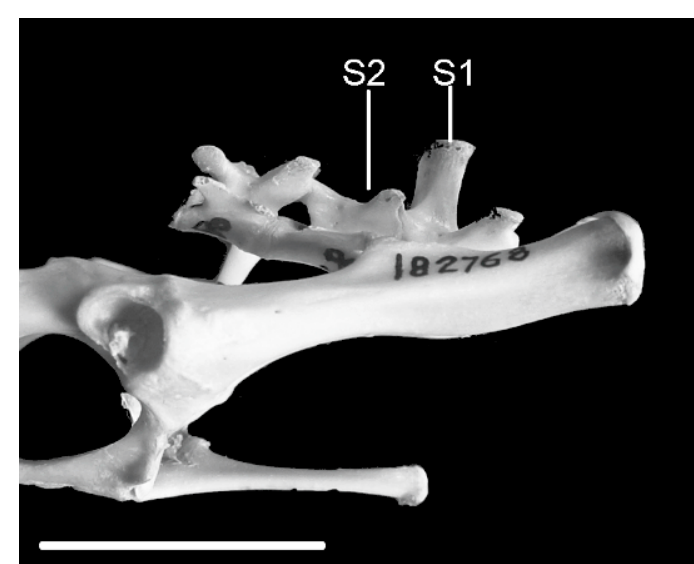

Marmosops incanus

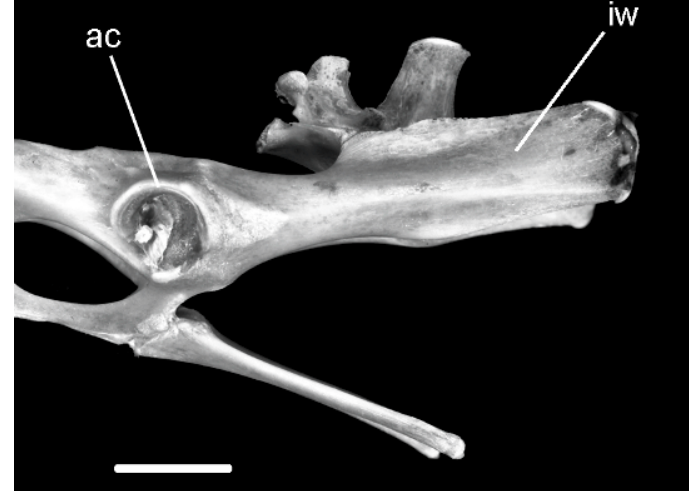

Metachirus nudicaudatus

Fig. 11. Marmosops incanus (MVZ 182768) and Metachirus nudicaudatus (AMNH 267009), right os coxae with sacral vertebrae in lateral view. In the sacral vertebrae of Marmosops incanus the spinous process is present only in S1 (ch. 40[0]), whereas in Metachirus the process is present in both vertebrae (ch. 40[1]), and the S1 spinous process is taller than the $\mathrm{S} 2$ spinous process (ch. 41[1]). In Metachirus, the acetabulum (ac) is close, deep, and with the dorsal part laterally extended (ch. 83[1]), and the iliac wing (iw) forms a large blade (ch. 84[1]). Scale bars: $10 \mathrm{~mm}$.

which move the tail in a transverse plane, in addition to muscles related to hindlimb movement (i.e. Mm. caudofemoralis, femoro-coccygeus, and semitendinosus caput dorsalis; Argot, 2002, 2003a). Most didelphid taxa treated herein show a caudal vertebra with the transverse process length similar to vertebral body craniocaudal length (see Argot, 2003a: fig. 10a), except for some taxa such as Glironia, Caluromys, Monodelphis theresa, Marmosa rubra, and Cryptonanus unduaviensis, where this kind of vertebra is absent. I only observed polymorphism in Marmosa robinsoni (coded $\{01\}$ ).

Character 44: Presence of spinous process in the first three caudal vertebrae: (0) spinous process absent; (1) spinous process only in first caudal vertebra; (2) spinous process in the first two caudal vertebrae; (3) spinous process present in the first three caudal vertebrae. The development of the spinous process on the anterior caudal vertebrae suggests development of the $\mathrm{m}$. multifidus caudae, an important extensor muscle of the tail implied in vertical movements (Muizon and Argot, 2003). The spinous process is absent on the anterior caudal vertebrae in Didelphis, Philander, Thylamys, Lestodelphys, Marmosops, and Cryptonanus. Only Lutreolina and Marmosa robinsoni show the spinous process just in the first caudal vertebra, and Monodelphis brevicaudata and Gracilinanus exhibit the spinous process in the first two caudal vertebrae. The remaining taxa have the spinous process in the first three caudal vertebrae. Because I observed intermediate conditions in the presence of the spinous process in the caudal vertebrae, this character is treated as ordered $(0 \leftrightarrow 1 \leftrightarrow 2 \leftrightarrow 3)$ in all analyses.

Character 45: Orientation of the spinous process in the three first caudal vertebrae: (0) spinous process in $\mathrm{Ca} 1-\mathrm{Ca} 3$ all vertical; (1) spinous process in $\mathrm{Ca} 1-\mathrm{Ca} 2$ vertical, and in $\mathrm{Ca} 3$ caudally oriented; (2) spinous process in Ca1 vertical, and in $\mathrm{Ca} 2-\mathrm{Ca} 3$ caudally oriented; (3) spinous process in $\mathrm{Ca} 1-\mathrm{Ca} 3$ caudally oriented. In those taxa with the spinous process present on the first three caudal vertebrae (i.e., state 3 in character 44), I observed variation related to the spinous process orientation. In some taxa, such as Monodelphis adusta, M. theresa, Marmosa rubra, M. mexicana, and Micoureus, the spinous processes are all vertical, but in Caluromys the two first are vertical, and the third one is caudally oriented. On the other hand, Chironectes and Metachirus have the second and third spinous process caudally 


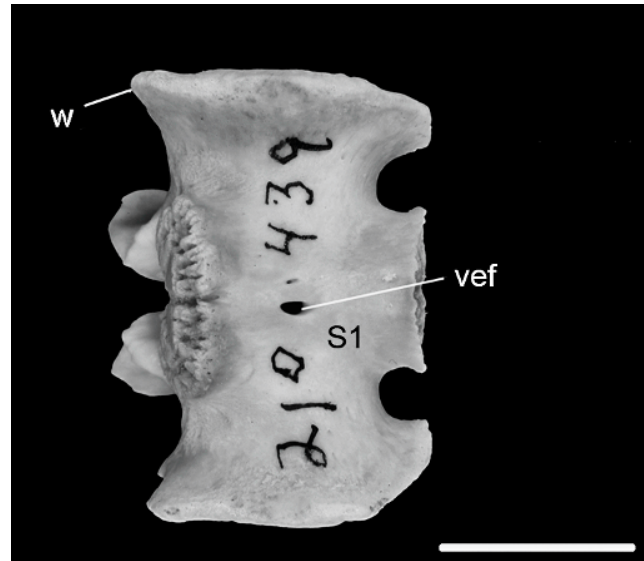

Didelphis marsupialis

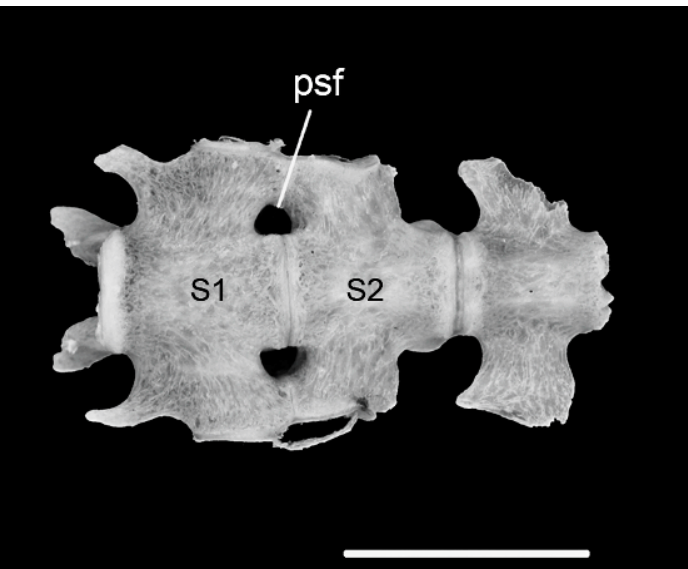

Caluromys philander

Fig. 12. Didelphis marsupialis (AMNH 210439 and Caluromys philander (AMNH 267001), sacral vertebrae in ventral view. A ventral foramen (vef) in the S1 body is present in Didelphis (ch. 42[1]), whereas in Caluromys it is absent (ch. 42[0]). Other abbreviations: psf, pelvic sacral foramen; w, wing. Scale bars: $10 \mathrm{~mm}$.

oriented. Only in Caluromysiops and Tlacuatzin do the first three caudal vertebrae have the spinous process caudally oriented. In all taxa that do not have a spinous process on all first three caudal vertebrae, this character is treated as inapplicable (coded "_"). Because I observed intermediate conditions on the orientation of the spinous process, this character is treated as ordered $(0 \leftrightarrow 1 \leftrightarrow 2$ $\leftrightarrow 3)$ in all analyses.

Character 46: Position of caudal vertebra with craniocaudal transverse process length similar to vertebral body length: (0) 3; (1) 4; (2) 5 ; (3) 6 . On this character, I refer to the position of the vertebral element mentioned in character 43. The position of the first vertebra with this characteristic is variable in the didelphid crown group. In Thylamys macrurus and Marmosops incanus, this feature is placed in Ca3, whereas in Lestodelphys and Monodelphis adusta it is in Ca4. Chironectes and Caluromysiops have this morphology on Ca6. Lastly, the position in Ca5 is the most common condition in didelphids. It is interesting to note the posterior position of this element in the semiaquatic Chironectes, which is indicative of the posterior extension of the $\mathrm{Mm}$. ischio-caudalis and abductor caudae dorsalis, both involved in lateral movements of the tail. All taxa that do not have this kind of vertebra (coding 0 in character 43) are treated as inapplicable (coded "-") for this character. No information is currently available about this character for Gracilinanus microtarsus (coded “'?"). Because I observed intermediate conditions on the position of this element, this character is treated as ordered $(0 \leftrightarrow 1 \leftrightarrow 2 \leftrightarrow 3)$ in all analyses.

Character 47: Foramen on the transverse process of the caudal vertebra referred in character 46: (0) absent; (1) present. The presence of a foramen in dorsal view on the transverse process of this particular vertebra is variable in didelphids. It is absent in Caluromysiops, Thylamys, Gracilinanus, and Monodelphis, but it is present in Philander frenatus, P. mcilhennyi, Lestodelphys, Metachirus, Chironectes, Micoureus, Marmosa mexicana, Marmosops impavidus, and $M$. noctivagus. In several taxa, such as Philander opossum, Didelphis, Lutreolina, Tlacuatzin, Marmosa robinsoni, M. murina, and Marmosops incanus, this character is treated as polymorphic (coded $\{01\}$ ) because of asymmetry. All taxa that do not have this kind of vertebra (coding 0 in character 43) are treated as inapplicable (coded "_,") for this character.

Character 48: Position (from S1) of the first caudal vertebra with articulation through 
vertebral body: (0) between 4 and 5; (1) between 5 and 6 ; (2) between 6 and 7. The caudal vertebrae exhibit two kinds of articulation: in the anterior portion of the tail, through pre- and postzygapophysis, similar to the sacral and lumbar vertebrae; and in the posterior portion, via the vertebral bodies and intervertebral discs. This joint configuration occurs with morphological modifications on the vertebral body (i.e., the vertebrae become longer, slender, and exhibit reduced apophyses). The articulation through intervertebral discs increases the flexibility of the posterior portion of the tail (Argot, 2003a). This simplified kind of articulation starts on different positions in the tail in different didelphids. The articulation between caudal vertebrae 4 and 5 occurs in Glironia, Tlacuatzin, Thylamys macrurus, Monodelphis adusta, M. theresa, and Cryptonanus unduaviensis. On the other hand, this articulation is placed between caudal vertebrae 5 and 6 in Philander, Metachirus, Didelphis, Lutreolina, Thylamys venustus, T. pallidior, T. pusillus, Lestodelphys, Monodelphis brevicaudata, Micoureus, Marmosa, Marmosops (except incanus), and Gracilinanus agilis. Lastly, in Caluromys, Caluromysiops, and Chironectes, the articulation is between caudal vertebrae 6 and 7. I found individual variation only in Marmosops incanus (coded $\{01\}$ ). No information is currently available about this character for Gracilinanus microtarsus (coded "'?"). Because I observed the intermediate condition in the position of the articulation through the vertebral body, this character is treated as ordered $(0 \leftrightarrow 1 \leftrightarrow 2)$ in all analyses.

\section{RIBS AND STERNUM}

Character 49: Ribs with anteroposterior extension: (0) present; (1) absent (fig. 5). Only in Caluromys and Caluromysiops do the ribs exhibit anteroposterior extension, which reduces the space filled by the intercostal muscles (Jenkins, 1970; Argot, 2003a). This morphology is also present in some primates, myrmecophagids, and dasypodids (Jenkins, 1970; MacPhee and Jacobs, 1986; Gebo, 1989). Rib morphology is indirectly related to the functionality of the vertebral column by altering mechanical properties of the thorax (Jenkins, 1970). The craniocaudal extension of the ribs increases the rigidity of the rib cage and reduces the flexibility provided by muscles and ligaments (Jenkins, 1970; Sargis, 2001; Argot, 2003a). Jenkins (1970) concluded that the expanded ribs increase the stability in the rib cage and the vertebral column as a whole. In arboreal forms such as Caluromys and Caluromysiops, this stability is necessary for locomotion during the bridging behavior (Jenkins, 1970; Sargis, 2001).

Character 50: Shape of the internal border of the first rib on distal half: (0) internal border notably curved; (1) internal border almost straight (fig. 13). In all didelphids, the first rib is short and robust since it is subject to tension forces, and it develops an especially strong connection with the manubrium (Klima, 1987). Some important muscles, such as $\mathrm{Mm}$. pectoralis, scalenus, subclavius, and serratus ventralis thoracic, attach on the manubrium and the first pair of ribs (Jenkins and Weijs, 1979). Among other functions, these muscles support the head and forequarters and transmit forces between forelimb and trunk (Argot, 2003a, 2003b). In some taxa, such as Caluromys, Caluromysiops, Thylamys, Marmosa murina, M. robinsoni, Marmosops, Monodelphis, Micoureus, Cryptonanus, and Lestodelphys, the internal border at the distal half of the first pair of ribs is notably curved. In contrast, in Didelphis, Metachirus, Philander, Lutreolina, Chironectes, Marmosa rubra, M. mexicana, and Gracilinanus agilis this element is almost straight. No information is currently available about this character for Gracilinanus microtarsus (coded “'?').

Character 51: Postmanubrium sternebrae morphology: (0) sternebrae not laterally compressed; (1) second sternebra laterally compressed; (2) second and third sternebrae laterally compressed. The body of the postmanubrium sternebrae appears laterally compressed in some taxa, which suggests an important function of the $\mathrm{m}$. pectoralis. None of the sternebrae is laterally compressed in Didelphis, Philander, Metachirus, Tlacuatzin, Marmosa mexicana, Marmosops incanus, M. noctivagus, M. impavidus, Monodelphis brevicaudata, M. adusta, Micoureus, Thylamys, Lestodelphys, and Gracilinanus. 


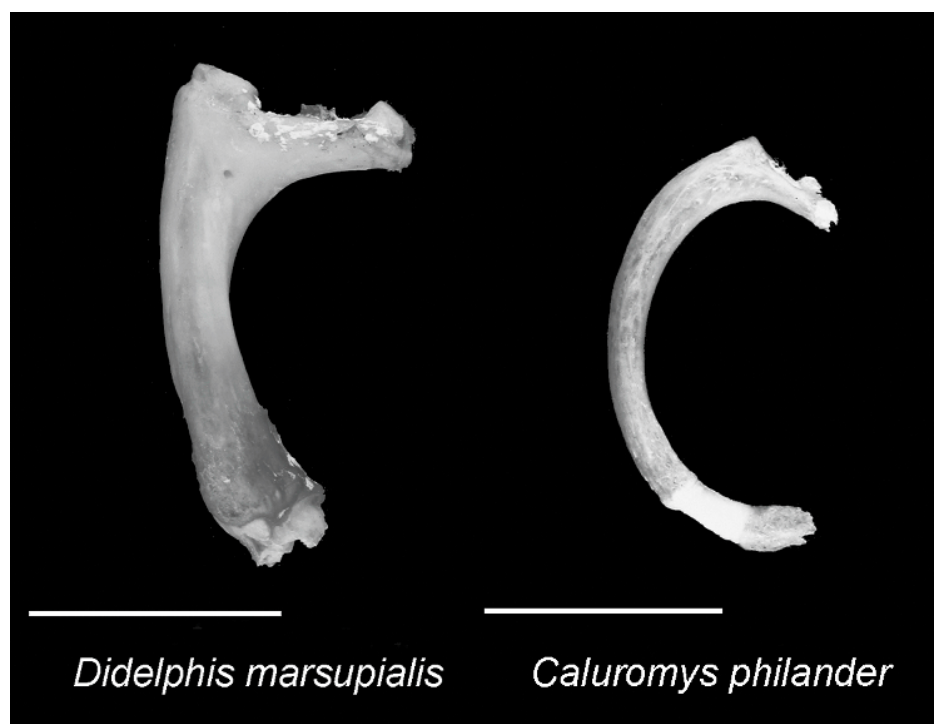

Fig. 13. Didelphis marsupialis (AMNH 23448) and Caluromys philander (AMNH 267001), first rib in anterior view. The internal border is notably curved in Caluromys (ch. 50[0]), whereas in Didelphis it is almost straight (ch. 50[1]). Scale bars: $5 \mathrm{~mm}$.

On the other hand, Lutreolina, Chironectes, Marmosa robinsoni, Marmosops pinheiroi, $M$. parvidens, Monodelphis theresa, and Cryptonanus exhibit the second sternebra laterally compressed. Lastly, a third condition is evident in Glironia, Caluromys, and Caluromysiops, where the second and third sternebrae are laterally compressed. No information is currently available about this character for Marmosa rubra and M. murina (coded “?'). Because I observed the intermediate condition on the sternebrae morphology, this character is treated as ordered $(0 \leftrightarrow 1$ $\leftrightarrow 2$ ) in all analyses.

Character 52: Development of keel in the manubrium: (0) keel not very developed; (1) keel very developed (fig. 14). Similarly to the laterally compressed sternebrae, a keeled manubrium is indicative of important function of the $\mathrm{m}$. pectoralis. The keel on the manubrium exhibits different levels of development in the taxa analyzed in this report. It is not very developed in Lutreolina, Chironectes, Tlacuatzin, Marmosa mexicana, M. robinsoni, M. murina, Monodelphis, Thylamys, Micoureus, Gracilinanus, and Cryptonanus. In contrast, the keel is notably developed in Caluromys, Metachirus, Didelphis, Philander, Marmosops, and Lestodelpys. A poly- morphic condition is present in Caluromysiops (coded $\{01\})$. No information is currently available about this character for Marmosa rubra (coded “?”').

\section{SCAPULA}

Character 53: Coracoid process shape: (0) hooklike process; (1) small process (fig. 15). This character is modified from Horovitz and Sánchez-Villagra (2003: ch. 36), as in this sample there are no taxa with the coracoid process absent. The development of the coracoid process is functionally important because this process is the point of origin of the Mm. coracobrachialis and biceps brachii, among other muscles. The first is involved in the extension and adduction of the shoulder joint (Taylor, 1974; Jenkins and Weijs, 1979; Evans, 1993; Sargis, 2002) and, according to Argot (2001), the morphology of this muscle depends on the species' habits. On the other hand, the $\mathrm{m}$. biceps brachii is implied in the flexion of the elbow (Taylor, 1974; Argot, 2001). In this sense, a large coracoid process provides a longer lever arm for the $\mathrm{m}$. biceps brachii, which is important during climbing in arboreal forms (Argot, 2001; Sargis, 2002). The coracoid process has a hooklike shape in 


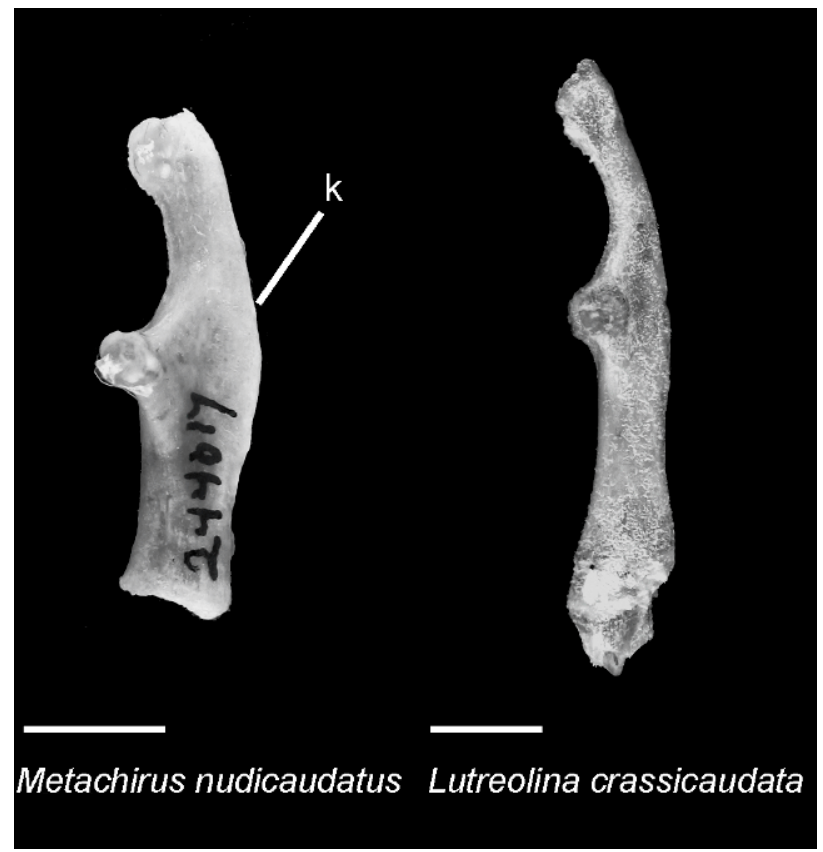

Fig. 14. Metachirus nudicaudatus (AMNH 244617) and Lutreolina crassicaudata (AMNH 33250), manubrium in lateral view. In Metachirus the keel (k) is well developed (ch. 52[1]), whereas in Lutreolina it is not prominent (ch. 52[0]). Scale bars: $5 \mathrm{~mm}$.

Glironia, Caluromys, Caluromysiops, Philander, Metachirus, Lutreolina, Chironectes, Marmosops parvidens, Marmosa rubra, M. robinsoni, and Tlacuatzin. On the other hand, the process is small in Lestodelphys, Didelphis, Thylamys, Micoureus, Monodelphis, Marmosa mexicana, M. murina, Marmosops (except M. parvidens), Cryptonanus, and Gracilinanus.

Character 54: Supraspinous fossa width: (0) less than one-fourth its length; (1) between one-fourth and one-half its length (fig. 16). This character is modified from Horovitz and Sánchez-Villagra (2003: ch. 40), since in the sample there are not taxa with the infraspinous fossa width more than one-half its length, and the character is treated as binary. The supraspinous fossa contains the m. supraspinatus, which originates in the greater tubercle of the humerus (see Taylor, 1974: fig. 5; Evans, 1993: fig. 6-45; Argot, 2001: fig 3a). The function of this muscle is to extend the shoulder joint and advance the limb (Evans, 1993), and it is important to stabilize and prevent collapse in the shoulder joint (Goslow et al., 1981). An additional function is to absorb part of the kinetic energy generated when the forelimbs contact the substrate at the end of a leap (Maynard Smith and Savage, 1956; Roberts, 1974). This could explain the highly developed supraspinous fossa in Metachirus, a specialized leaper among didelphids (Argot, 2001). Most of the didelphids analyzed herein exhibit condition 1, except for some taxa such as Lestodelphys, Chironectes, Monodelphis, and Hyladelphys, where the spinous fossa width is less than one-fourth its length.

Character 55: Scapular spine width at neck level: (0) subequal to infraspinous fossa; (1) wider than infraspinous fossa. This character is modified from Horovitz and SánchezVillagra (2003: ch. 41) since in this sample there are no taxa with a scapular spine that is narrower than the infraspinous fossa at the neck level, so the character is treated as binary. This section of the scapular spine is the area of insertion of some muscles involved in limb flexion, such as the $\mathrm{m}$. omotransversarius (Taylor, 1974) and the pars acromialis of $\mathrm{m}$. trapezius (MannFischer, 1953; Jenkins and Weijs, 1979), 


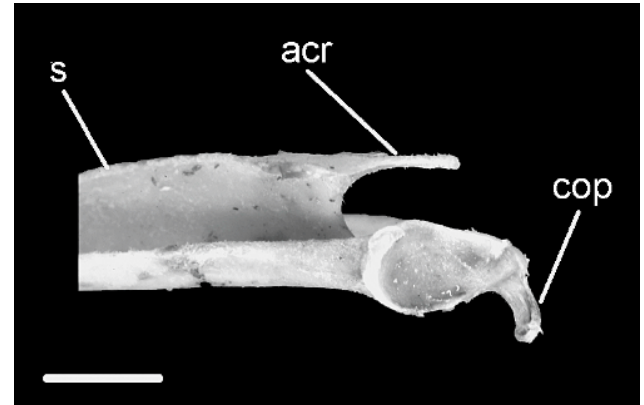

\section{Metachirus nudicaudatus}

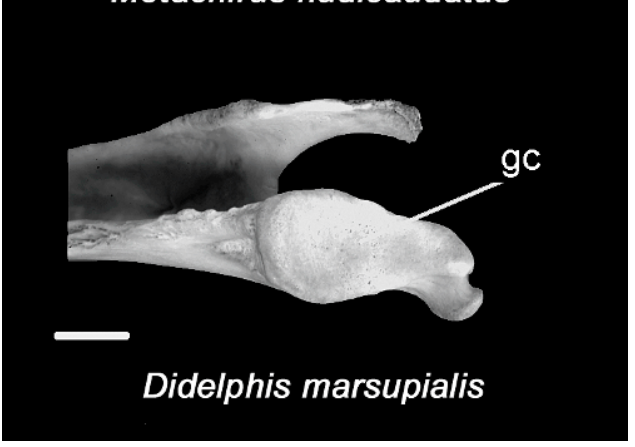

Fig. 15. Metachirus nudicaudatus (AMNH 244617) and Didelphis marsupialis (AMNH 210439), proximal area of scapula in ventrocaudal view. In Metachirus, the coracoid process (cop) shape is a hook-like process (ch. 53[0]), whereas in Didelphis this process is small (ch. 53[1]). Other abbreviations: acr, acromion; gc, glenoid cavity; s, spine. Scale bars: $5 \mathrm{~mm}$.

which exert an anteriorly directed force to move this area craniodorsally, elevating the limb and moving it forward (Argot, 2003a, 2003b; Evans, 1993; Larson, 1993). Most of the didelphids show a scapular spine that is wider than the infraspinous fossa, except for Caluromys, Didelphis, and Metachirus, where both structures are subequal.

Character 56: Infraspinous/supraspinous fossa width at neck level: (0) infraspinous fossa narrower; (1) subequal; (2) supraspinous fossa narrower. This character was described by Horovitz and Sánchez-Villagra (2003: ch. 42), and it exhibits significant variation in the didelphid crown group analyzed here. Both fossae are subequal in Philander, Didelphis, Metachirus, Monodelphis, and Gracilinanus agilis. A supraspinous fossa narrower than the infraspinous fossa is present in Glironia, Caluromys, Caluromy- siops, Lestodelphys, Lutreolina, Chironectes, Tlacuatzin, Thylamys pallidior, T. pusilla, T. venustus, Micoureus, Marmosa, Marmosops, Cryptonanus unduaviensis, and Gracilinanus microtarsus. Lastly, a narrower infraspinous fossa is present only in Thylamys macrurus and Marmosa rubra. Because I observed an intermediate condition in the relative width of the supra- and infraspinous fossae, this character is treated as ordered $(0 \leftrightarrow 1 \leftrightarrow 2)$ in all analyses.

Character 57: Caudal angle: (0) acute; (1) rounded (fig. 16). The caudal angle shape of the scapula is related to the function of $\mathrm{Mm}$. serratus and rhomboideus, which rotate the scapula in such a way that the vertebral border is pulled posteroventrally. The glenoid cavity is forced craniodorsally by the action of the m. omotransversarius (Maynard Smith and Savage, 1956; Taylor, 1974; Jenkins and Weijs, 1979; Larson, 1993; Argot, 2001; Muizon and Argot, 2003; see comments on character 55). The caudal angle is also the site for the attachment of $\mathrm{m}$. teres major (MannFischer, 1956; Jenkins, 1970; Taylor, 1974; Jenkins and Weijs, 1979), whose function is to flex the shoulder joint and move the humerus backwards (Maynard Smith and Savage, 1956; Taylor, 1974; Evans, 1993). The caudal angle of the scapula is acute in most taxa, such as Glironia, Caluromys, Caluromysiops, Philander, Lutreolina, Chironectes, Tlacuatzin, Thylamys venustus, $T$. macrurus, Monodelphis, Micoureus, Marmosa, Marmosops, Lestodelphys, Cryptonanus, and Gracilinanus. On the other hand, the caudal angle is rounded in Didelphis, Metachirus, and Thylamys pallidior. Individual variation is observed only in the samples of Thylamys pusillus and Marmosops noctivagus (coded $\{01\}$ ).

Character 58: Scapular notch extension: (0) extended less than half of the scapula; (1) extended to half of the scapula or beyond (fig. 16). An extended scapular notch determines the shape for the area of origin of the $\mathrm{m}$. supraspinatus. The scapulae in living opossums exhibit two morphological types (Argot, 2001: fig 2), related to their rotational mode. These two types are conditioned by the development of the supraspinous and infraspinous fossae, as well as the scapular notch extension. The scapular notch is 


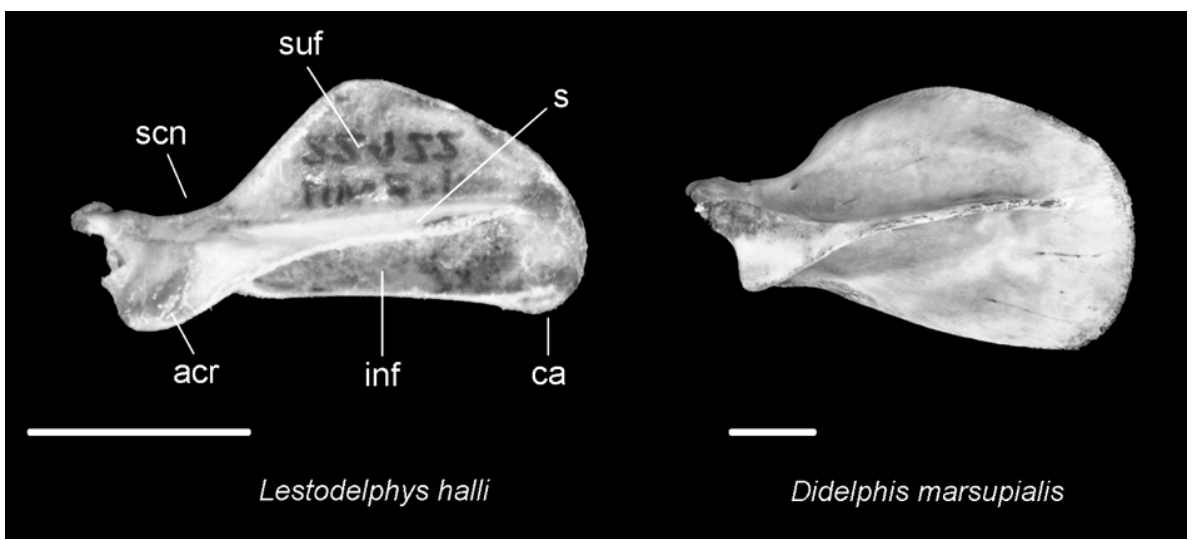

Fig. 16. Lestodelphys halli (UWZM 22422) and Didelphis marsupialis (AMNH 210439), left scapula in lateral view. In Lestodelphys the supraspinous fossa (suf) width is less than one-fourth its length (ch. 54[0]) and the caudal angle (ca) is acute (ch. 57[0]), whereas in Didelphis the supraspinous fossa width is between one-fourth and one-half its length (ch. 54[1]) and the caudal angle is rounded (ch. 57[2]). Note the scapular notch (scn) in Didelphis, extended to less than half of the scapula (ch. 58[0]), whereas in Lestodelphys it is extended to the middle of the scapula (ch. 58[1]). Other abbreviations: acr, acromion; inf, infraspinous fossa, s, spine. Scale bars: $10 \mathrm{~mm}$.

extended less than half of the scapula in Philander, Didelphis, Metachirus, Lutreolina, Chironectes, and Marmosops noctivagus. Alternatively, the scapular notch is extended beyond half of the scapula in Glironia, Caluromys, Caluromysiops, Lestodelphys, Tlacuatzin, Thylamys, Monodelphis, Marmosa, Marmosops impavidus, M. incanus, Cryptonanus, and Gracilinanus.

\section{Humerus}

Character 59: Medial relief for $\mathrm{m}$. teres major: (0) absent; (1) present. This character was defined by Horovitz and Sánchez-Villagra (2003: ch. 43). The relief for this muscle is absent in several species, such as Hyladelphys, Didelphis albiventris, D. virginiana, Metachirus, Lutreolina, Chironectes, Thylamys venustus, T. macrurus, Monodelphis, Micoureus, Marmosops parvidens, and $M$. impavidus. In contrast, the relief is apparent in Glironia, Caluromys, Caluromysiops, Philander, Lestodelphys, Tlacuatzin, Thylamys pallidior, T. pusillus, Marmosa, Marmosops noctivagus, M. incanus, M. pinheiroi, Gracilinanus, and Cryptonanus. I found a polymorphic condition in Didelphis marsupialis, Philander opossum, Micoureus paraguayanus, and Marmosa robinsoni (coded $\{01\}$ ).
Character 60: Capitulum shape: (0) spherical; (1) cylindrical (fig. 17). This character was defined by Horovitz and Sánchez-Villagra (2003: ch. 45). The capitulum articulates with the proximal radial head, and its shape is critical for arm movement. The spherical shape of the capitulum allows for a freer movement on the proximal radial head, during pronation-supination movements. The capitulum is spherical in several taxa, such as Glironia, Caluromys, Caluromysiops, Philander mcilhennyi, Marmosa rubra, Tlacuatzin, Thylamys venustus, T. pallidior, $T$. pusillus, Monodelphis, Micoureus regina, Marmosa robinsoni, M. mexicana, Marmosops impavidus, Cryptonanus unduaviensis, and Gracilinanus microtarsus. In contrast, it is cylindrical in Philander franatus, P. opossum, Lestodelphys, Didelphis, Metachirus, Lutreolina, Chironectes, Thylamys macrurus, Marmosa murina, Marmosops noctivagus, $M$. incanus, M. parvidens, and Gracilinanus agilis. Individual variation is observed in Marmosops pinheiroi, and Micoureus demerarae (coded $\{01\}$ ).

Character 61: Olecraneon fossa: (0) absent; (1) present; (2) present and large. This character is interpreted in the same way as by Horovitz and Sánchez-Villagra (2003: ch. 47), although the olecraneon foramen is not 


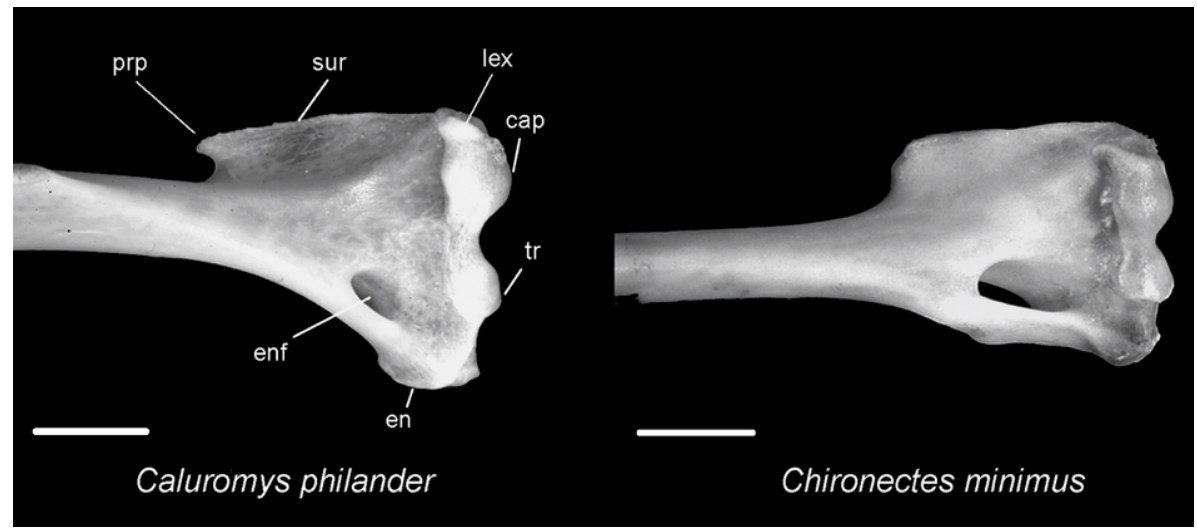

Fig. 17. Caluromys philander (AMNH 267001) and Chironectes minimus (AMNH 212909), distal portion of left humerus in anterior view. The capitulum (cap) in Caluromys is spherical in shape (ch. 60[0]), whereas in Chironectes it is cylindrical (ch. 60[1]). Note the more developed proximal extension of the capitulum in Caluromys relative to the proximal extension of the trochlea (tr) (ch. 63[1]). In both species there is a lateral extension (lex) of the capitulum (ch. (64[1]). In Caluromys, a proximal process (prp) in the supinator ridge (sur) is present (ch. 67[1]), whereas in Chironectes it is absent (ch. 67[0]). Other abbreviations: enf, entepicondylar foramen; en, entepicondyle. Scale bars: $5 \mathrm{~mm}$.

observed in the sample. In the arm movement, the depth and size of the olecraneon fossa is important in the extension of the elbow joint. A deep olecraneon fossa allows for a maximum extension of the ulna, since the proximal part of the ulnar trochlear notch strikes the humeral olecraneon fossa during the maximum arm extension. An evident olecraneon fossa is absent only in Caluromysiops and Marmosa mexicana. In most didelphid groups a fossa is present, but only Metachirus exhibits a notably large fossa. Because I observed an intermediate condition in the development of the olecraneon fossa, this character is treated as ordered $(0 \leftrightarrow 1 \leftrightarrow 2)$ in all analyses.

Character 62: Extension of the deltopectoral crest: (0) restricted to the proximal half; (1) reaching distal half. This character was described by Horovitz and Sánchez-Villagra (2003: ch. 50). Both parts of the m. deltoideus (i.e., pars clavicularis and pars acromialis) meet along the deltopectoral crest (Argot, 2001). A distal extension of the deltoid crest increases the length of the muscular fibers, emphasizing the function of flexion of the shoulder joint. Most didelphid taxa analyzed herein exhibit the deltoid crest restricted to the proximal half of the humerus. Nonetheless, in some terrestrial and generalist forms, such as Philander, Didelphis, Metachirus,
Lutreolina, and Monodelphis, the deltoid crest reaches the distal half. Individual variation is observed in Gracilinanus agilis and Micoureus paraguayanus (coded $\{01\}$ ).

Character 63: Proximal extension of capitulum and trochlea: (0) equal extension; (1) longer proximal extension of capitulum (fig. 17). This character was modified from Horovitz and Sánchez-Villagra (2003: ch. 52), since in the sample there are no taxa with a longer extension of the trochlea, and the character is treated as binary. The humeral trochlea, capitulum, proximal radial head, and the trochlear notch of the ulna are involved in flexion/extension movements in the elbow joint (Taylor, 1974; Gebo, 1989; Argot, 2001; Szalay and Sargis, 2001; Muizon and Argot, 2003). A proximal extension of the capitulum allows for a close angle of flexion in the elbow joint. Most didelphid groups exhibit equal proximal extension of the capitulum and trochlea, except for Glironia, Caluromys, and Caluromysiops, where there is a longer proximal extension of the capitulum. Individual variation is present in Chironectes and Gracilinanus agilis (coded $\{01\}$ ).

Character 64: Capitulum lateral extension: (0) absent; (1) present (fig. 17). The character is interpreted in the same way as in Horovitz and Sánchez-Villagra (2003: ch. 56). Accord- 
ing to Argot (2001: figs. 4a, 13d), the m. supinator brevis originates in this area, inserting in the proximal portion in the anterior side of the radius. This muscle is involved in the supination of the paw, so the palmar surface faces medially (Evans, 1993). All large opossums show a lateral extension of the capitulum, which is absent in mouse opossums.

Character 65: Shaft curvature: (0) straight; (1) curved. This character is interpreted in the same way as in Horovitz and SánchezVillagra (2003: ch. 57). Among the didelphid groups, just Glironia, Caluromys, and Caluromysiops display an almost straight humerus.

Character 66: Olecraneon fossa depth: (0) shallow; (1) deep. This character is independent from character 61 , since here I specifically evaluate the variation existing in the olecraneon fossa depth in the taxa where it is present (coded as 1 and 2 in character 61). As mentioned above, the depth and enlargement of the olecraneon fossa are important in the extension of the elbow joint, because a deep fossa allows a major angle of extension in the joint. In this sense, the olecraneon fossa exhibits different depth in the didelphid group. It is not very deep in Glironia, Caluromys, Lestodelphys, Tlacuatzin, Thylamys pallidior, $T$. venustus, $T$. macrurus, Micoureus, Marmosops impavidus, M. noctivagus, Cryptonanus unduaviensis, and Gracilinanus. On the other hand, some taxa such as Philander, Didelphis virginiana, Metachirus, Lutreolina, Chironectes, Monodelphis, Marmosops pinheiroi, M. parvidens, and $M$. incanus exhibit a deep fossa. Individual variation is common in several taxa such as Didelphis albiventris, D. marsupialis, Marmosa robinsoni, M. murina, and Thylamys pusillus (coded \{01\}). Caluromysiops and Marmosa mexicana are inapplicable in this character (coded "-"), because the olecraneon fossa is absent (coding 0 in character 61).

Character 67: Proximal process on the supinator ridge: (0) absent; (1) present (fig. 17). Some muscles involved in the flexion and extension of the elbow joint originate on the supinator ridge. The presence of a posterior process on the supinator ridge increases the area for the $\mathrm{Mm}$. triceps brachii caput laterale and the $\mathrm{m}$. brachiora- dialis. The former inserts on the olecraneon of the ulna and produces the extension of elbow joint, and the $\mathrm{m}$. brachioradialis inserts on the distal portion of the medial side of radius, and it is involved in the rotation of the radius dorsolaterally (Jenkins and Weijs, 1979; Evans, 1993). A small proximal process on the supinator ridge is present in Glironia, Caluromys, Caluromysiops, Marmosa rubra, Marmosops parvidens, and Lestodelphys. Individual variation is observed in Marmosa robinsoni (coded $\{01\}$ ).

Character 68: Deltopectoral crest notably developed: (0) absent; (1) present. As mentioned above, this crest is important for the insertion of the $\mathrm{m}$. deltoideus. This character is independent of character 62 , because here I focus on the variation existing on the development but not on the extension of the deltopectoral crest. The crest is notably developed in Glironia, Caluromys, Caluromysiops, Didelphis, Metachirus, Chironectes, Monodelphis, Micoureus demerarae, Marmosa murina, M. rubra, and Marmosops. On the other hand, the crest is not well developed in Philander, Lestodelphys, Lutreolina, Tlacuatzin, Thylamys, Micoureus regina, Marmosa mexicana, Cryptonanus unduaviensis, and Gracilinanus microtarsus. Individual variation is observed in Marmosa robinsoni and Gracilinanus agilis (coded $\{01\}$ ).

Character 69: Entepicondyle mesial expansion: (0) absent; (1) barely expanded, approximately the same width as the trochlea; (2) notably expanded, wider than the trochlea (fig. 17). Most muscles moving the wrist and the hand originate on the humeral epicondyles (Taylor, 1974; Argot, 2001). The entepicondyle, also called the medial epicondyle, is the area where the Mm. flexor carpi radialis and the flexor digitorum profundus and superficialis originate. The former inserts on the palmar side of metacarpals II and III through two tendons. The flexor profundus and superficialis are involved in digit flexion (Abdala et al., 2006). The development of the entepicondyle is associated with powerful flexors, which are necessary for climbing habits (Taylor, 1974). However, the entepicondyle is a particularly expanded process only in some taxa, which are not always associated with arboreal locomotion, such as Lestodelphys, Micoureus demerarae, Mar- 
mosa murina, Marmosops impavidus, and Gracilinanus agilis. Since I observed an intermediate condition in the development of the entepicondyle, this character is treated as ordered $(0 \leftrightarrow 1 \leftrightarrow 2)$ in all analyses.

Character 70: Depth of bicipital groove: (0) absent; (1) present but shallow; (2) very deep. The bicipital groove is filled by the $\mathrm{m}$. biceps brachii, originating from the coracoid process in the scapula. In arboreal taxa this muscle is well developed, having an important role in pulling the body up when climbing (Taylor, 1974; Argot, 2001). The bicipital groove is absent in Metachirus, Philander, Didelphis, Lutreolina, Chironectes, and Marmosa mexicana. This groove is very deep in Glironia, Caluromys, Caluromysiops, and Marmosa rubra. Polymorphism is evidenced in Micoureus demerarae and $M$. paraguayanus, as in those taxa some specimens show a very deep bicipital groove (coded $\{12\})$. Because I observed an intermediate condition in the depth of the bicipital groove, this character is treated as ordered ( 0 $\leftrightarrow 1 \leftrightarrow 2$ ) in all analyses.

\section{RADIUS AND ULNAE}

Character 71: Ulna, shape of the proximal posterior border: (0) curved; (1) straight (fig. 18). In some taxa the posterior border of the ulna presents a proximal curvature, a product of the combinations of tractions, produced by flexors and extensors of the elbow joint (Argot, 2001). In this particular area is inserted the $\mathrm{m}$. triceps brachii, whose function is to extend the elbow joint (Evans, 1993). Some authors linked this curvature to arboreal habits (Walker, 1974; Argot, 2001). Although most taxa have the ulnar proximal posterior border curved, in Metachirus, Didelphis, and Lutreolina, this section of the ulna is almost straight.

Character 72: Ulna, olecraneon shape: (0) olecraneon strong, short, and wide; (1) olecraneon slender (fig. 19). The short olecraneon would not only be related to the reduction of power, but also to the increment of the speed of movement. This was reasonably interpreted as an adaptation for arboreal habits, since the extension of the elbow does not require power but rapidity to grasp a support in arboreal locomotion (Muizon

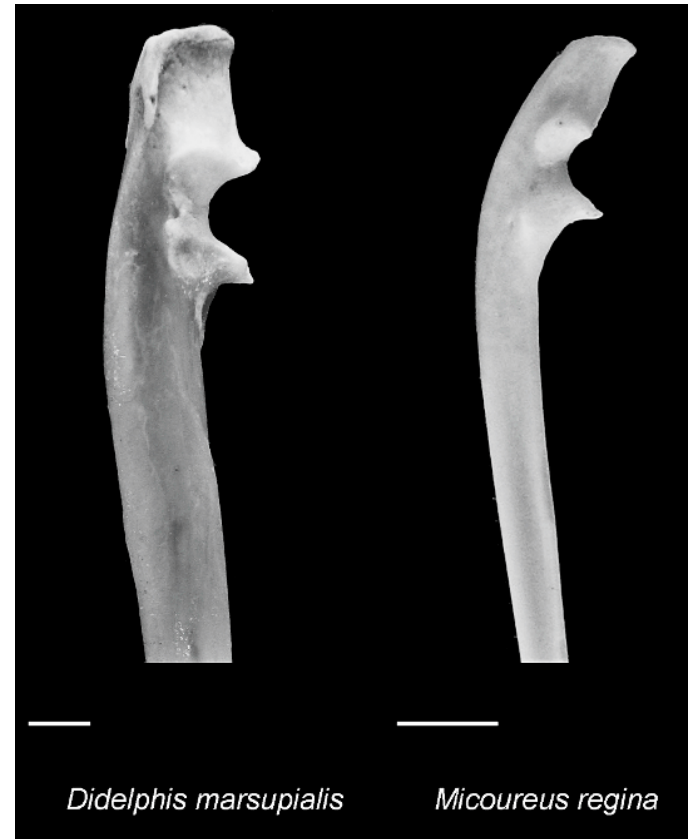

Fig. 18. Didelphis marsupialis (AMNH 210427) and Micoureus regina (AMNH 48757), proximal portion of ulna in lateral view. The caudal border is straight in Didelphis (ch. 71[1]), whereas it is strongly curved in Micoureus (ch. 71[0]). Scale bars: $10 \mathrm{~mm}$.

and Argot, 2003). However, although continuous variation in the development of the olecraneon is observed, it is possible to identify a different condition in the short and wide olecraneon exhibited by Metachirus, Hyladelphys, and Marmosops parvidens.

Character 73: Ulna, crest on the anterior side, for the origin of $\mathrm{Mm}$. pronator quadratus and flexor digitorum profundus: (0) absent; (1) present. Except for Metachirus and Didelphis, all groups analyzed herein present an evident crest on the anterior side of the ulna for the origin of $\mathrm{Mm}$. flexor digitorum profundus and pronator quadratus. These muscles are involved in the prehensility of the manus and in maintaining the integrity of the antebrachium near the carpus (Argot, 2001).

Character 74: Ulna, extension of the fossa for the exterior ligament: (0) absent; (1) restricted to olecraneon; (2) extended to the trochlear notch; (3) extended beyond the 


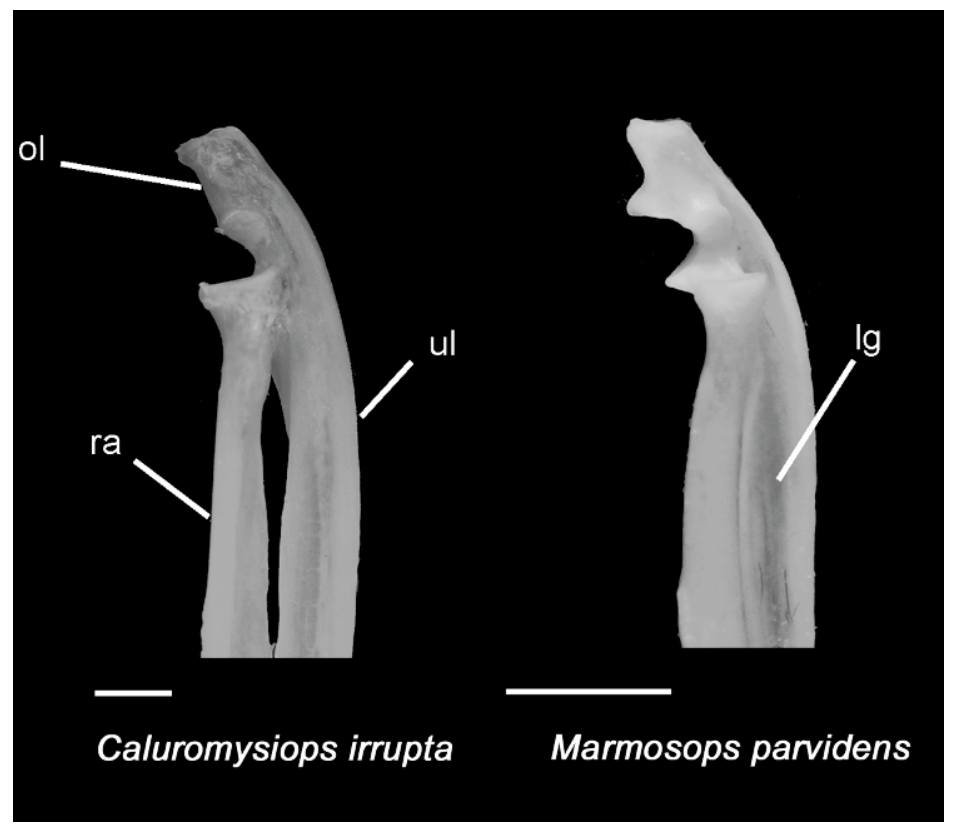

Fig. 19. Caluromysiops irrupta (AMNH 244364) and Marmosops parvidens (AMNH 267348), proximal portion of ulna (ul) and radius (ra) in lateral view. The olecraneon (ol) is short and wide in Marmosops (ch. $72[0]$ ), whereas it is proportionally longer and more slender in Caluromysiops (ch. 72[1]). In both species, the longitudinal groove (lg) for Mm. anconeus and abductor pollicis longus (ch. 77[2]) is well developed. Scale bars: $5 \mathrm{~mm}$.

trochlear notch (fig. 20). This fossa can be deep in some taxa, and it is part of the extensive surface for the origin of $\mathrm{m}$. flexor digitorum profundus (Muizon and Argot, 2003). It has also been associated with arboreal habits, because of the importance of this muscle for grasping during locomotion (Muizon and Argot, 2003) and food manipulation. In didelphids, the fossa for the exterior ligament is evident in mesial view, and it shows different levels of distal extension. It is restricted to the olecraneon only in Metachirus, whereas it is extended to the trochlear notch in Caluromysiops, Philander mcilhennyi, Didelphis, Lutreolina, Chironectes, Marmosa, Thylamys, Monodelphis brevicaudata, M. adusta, Micoureus regina, Lestodelphys, and Marmosops (except $M$. parvidens, where it is absent). The fossa is extended beyond the trochlear notch in Glironia, Caluromys, Philander opossum, $P$. frenatus, Tlacuatzin, Monodelphis theresa, Micoureus demerarae, M. paraguayanus, and Gracilinanus. Since I observed an intermediate condition in the extension of the fossa for the exterior ligament, this character is treated as ordered $(0 \leftrightarrow 1 \leftrightarrow 2)$ in all analyses.

Character 75: Ulna, mesial extension of the greater sigmoid cavity: (0) not extended; (1) notably extended, beyond the level of the anconeal process (fig. 21). The humeral trochlea rests in the greater sigmoid cavity and serves like a pivot during the elbow joint movement. Functionally, a mesial extension of the greater sigmoid cavity contributes to the safety of the elbow joint. In general, didelphids have the greater sigmoid cavity notably extended to the mesial side. However, in some taxa such as Metachirus, Philander mcilhennyi, Marmosops parvidens, and Lestodelphys, the cavity does not have a particular mesial extension.

Character 76: Ulna, development of the anconeal process: (0) present but not very developed; (1) well developed (fig. 21). The anconeal process is part of the insertion of $\mathrm{m}$. anconeus, which is involved, together with $\mathrm{m}$. triceps brachii, in the extension of the elbow joint (Jenkins and Weijs, 1979; Evans, 1993). The process is well developed in Metachirus, 


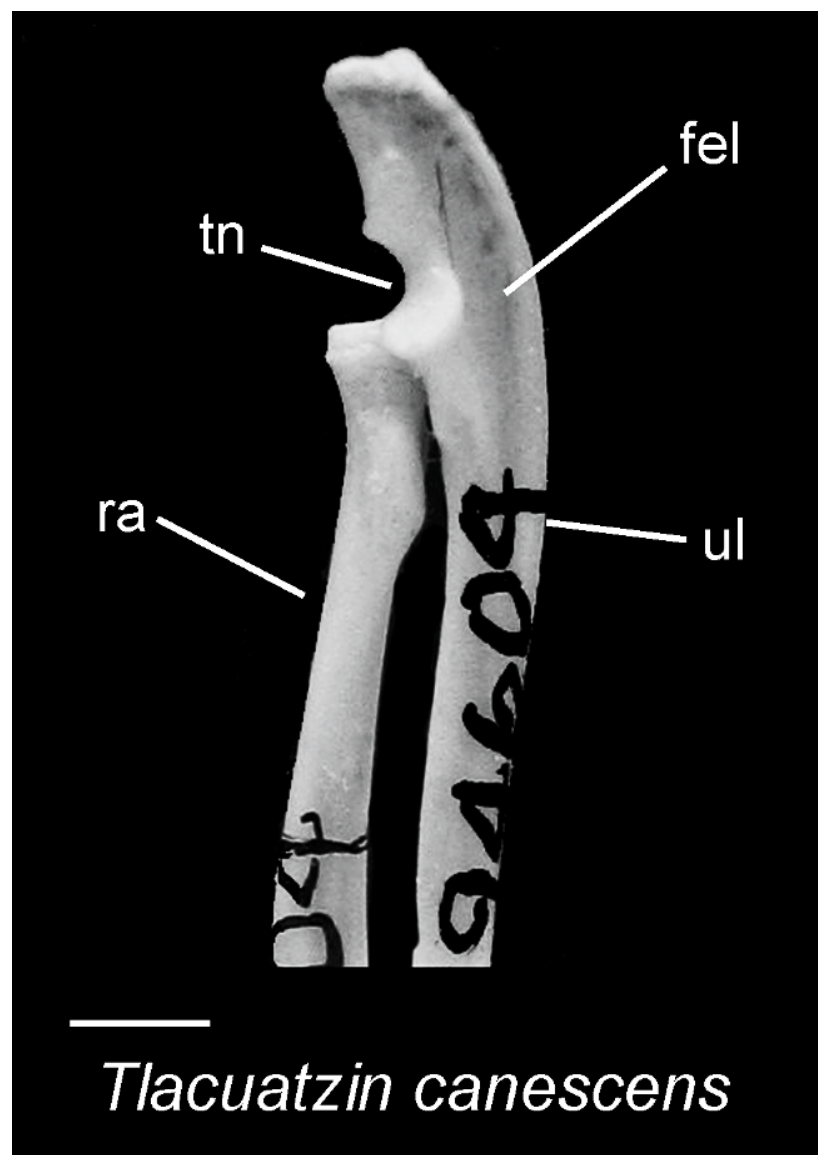

Fig. 20. Tlacualtzin canescens (UMMZ 94604), proximal portion of ulna (ul) and radius (ra) showing the fossa for exterior ligament (fel) extended beyond the trochlear notch (tn) (ch. 74[3]). Scale bar: $5 \mathrm{~mm}$.

Philander opossum, Didelphis, Lutreolina, Chironectes, Thylamys, Monodelphis adusta, Lestodelphys, Marmosops incanus, M. parvidens, and Gracilinanus agilis.

Character 77: Ulna, longitudinal groove in lateral surface for the insertion of $\mathrm{Mm}$. abductor pollicis longus and anconeus: (0) absent; (1) present and shallow; (2) present and notably deep (fig. 19). In the lateral surface of the ulna there is an area for the insertion of $\mathrm{m}$. anconeus and origin of the $\mathrm{m}$. abductor pollicis longus, both related to the extension of the elbow joint (Evans, 1993). According to Argot (2001), those muscles are well developed in arboreal forms. However, some arboreal forms, such as Caluromys, do not have a well-developed groove when it is compared to Caluromysiops. The groove in the lateral surface is absent in Thylamys,
Monodelphis brevicaudata, M. adusta, and Marmosa mexicana. It is present though not very deep in Glironia, Metachirus, Caluromys, Philander, Didelphis, Lutreolina, Chironectes, Tlacuatzin, Marmosa rubra, Monodelphis theresa, Micoureus, Lestodelphys, Marmosops (except M. parvidens), Cryptonanus, and Gracilinanus microtarsus. This groove is very deep only in Caluromysiops and Marmosops parvidens, indicating a very developed $\mathrm{m}$. anconeus. Polymorphism is observed in Marmosa murina, Micoureus paraguayanus, and Gracilinanus agilis (coded $\{01\}$ ). Because I observed an intermediate condition in the development of the longitudinal groove in the lateral surface, this character is treated as ordered $(0 \leftrightarrow 1 \leftrightarrow 2)$ in all analyses.

Character 78: Ulna, lateral extension of the coronoid process: (0) absent; (1) notably 


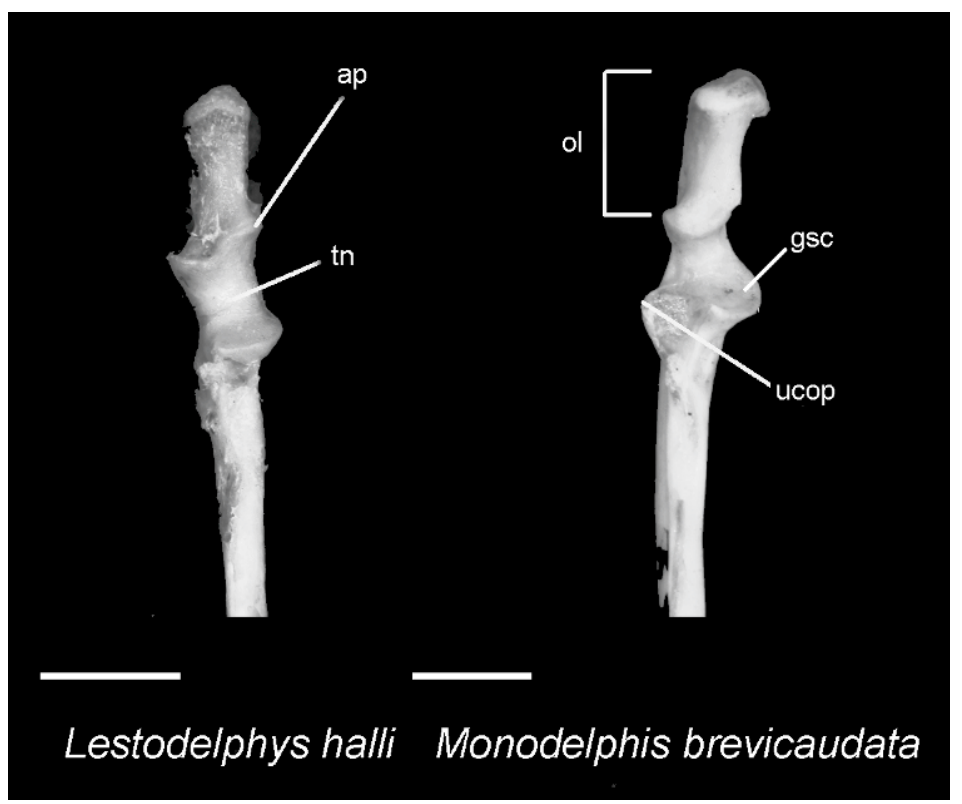

Fig. 21. Lestodelphys halli (UWZM 22422) and Monodelphis brevicaudata (AMNH 257203), proximal part of right ulna, anterior surface. In Monodelphis, the anconeal process (ap) is poorly developed (ch. 76[0]) and the ulnar coronoid process (ucop) is well developed in the lateral side (ch. 78[1]). In contrast, in Lestodelphys the anconeal process is well developed (ch. 76[1]) and the ulnar coronoid process is not very developed on the lateral side (ch. 78[0]). The greater sigmoid cavity (gsc) is mesially extended in Monodelphis (ch. 75[1]), whereas in Lestodelphys it is not mesially extended (ch. 75[0]). Other abbreviations: ol, olecraneon; tn, trochlear notch. Scale bars: $5 \mathrm{~mm}$.

extended, beyond the level of the trochlear notch (fig. 21). The coronoid process on the lateral side appears notably developed only in Monodelphis, Marmosops parvidens, and $M$. incanus.

Character 79: Radius, lateral compression: (0) radius little or not laterally compressed; (1) radius notably laterally compressed. A laterally compressed radius has been functionally associated to a major flexibility during pronation-supination movements (Oxnard, 1963; Walker, 1974). The radius is notoriously compressed in some species such as Glironia, Caluromysiops, Caluromys lanatus, Lutreolina, Monodelphis, Marmosa rubra, Micoureus demerarae, Marmosops parvidens, and Lestodelphys.

Character 80: Radius, shape of articular facet for humerus: (0) circular; (1) anteroposteriorly compressed. This character was defined by Horovitz and Sánchez-Villagra (2003: ch. 61). The radial head has some functional implications in pronation-supination movements (Argot, 2001). In arboreal forms, the circular radial head allows a wide potential range of pronation-supination movement. A radial head craniocaudally compressed is indicative of a more stable radio-capitullum joint, which is observed in scansorial forms, different from the circular shape observed in arboreal forms (MacLeod and Rose, 1993). The articular facet is anteroposteriorly compressed only in Philander, Marmosa rubra, and Chironectes.

Character 81: Radius, development of the bicipital tuberosity: (0) scarcely marked; (1) very developed (fig. 22). The bicipital tuberosity of the radius is the site of insertion of $\mathrm{m}$. biceps brachii. According to Argot (2001), the bicipital tuberosity is more developed in arboreal forms. I found a well-developed bicipital tuberosity in Glironia, Caluromys, Caluromysiops, Didelphis, Lutreolina, Marmosops, Lestodelphys, Cryptonanus, and Gracilinanus agilis. On the other hand, the tuberosity is scarcely marked in Metachirus, Philander, Chironectes, Tlacuatzin, Marmosa, Monodelphis, Thylamys, Micoureus, and Gracilinanus microtarsus. 


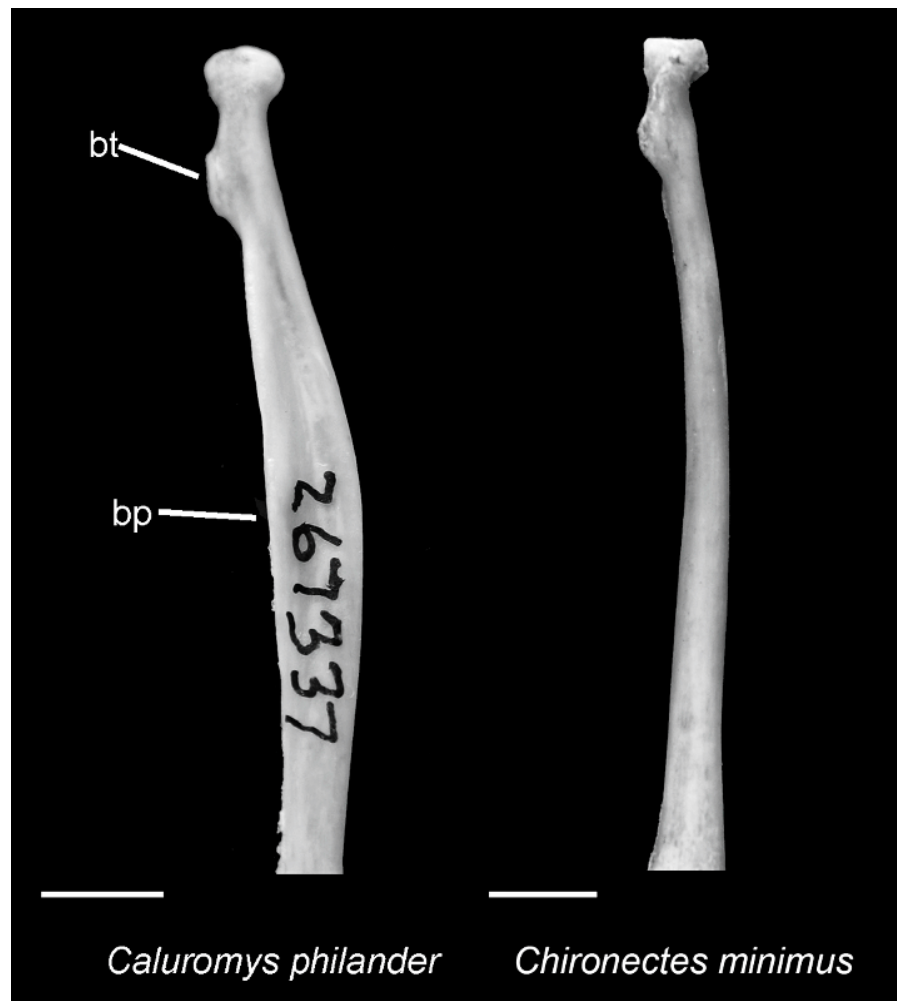

Fig. 22. Caluromys philander (AMNH 267337) and Chironectes minimus (AMNH 148720), radius in lateral view showing the well-developed bicipital tuberosity (bt) (ch. 81[1]) and the bony plate (bp) on the caudal portion (ch. 82[1]) in Caluromys, whereas in Chironectes the bicipital tuberosity is small (ch. 81[0]) and the bony plate is absent (ch. 82[0]). Scale bar: $10 \mathrm{~mm}$.

Character 82: Radius, presence of a thin bony plate extended posterolaterally along the diaphysis: (0) absent; (1) present (fig. 22). Only in Caluromys, Caluromysiops, and Lestodelphys is there a thin bony plate on the diaphysis of the radius. According to Argot (2001), this structure reinforces the origin for the $\mathrm{Mm}$. flexor digitorum profundus and pollicis longus, both important in the prehensility of the manus. Although this bony plate and a prounced curvature of radius have been associated to arboreal habits (Argot, 2001; Lanyon, 1980), the morphology of the radius in the terrestrial Lestodelphys suggests an important capacity for manipulating prey.

\section{Pelvis And Epipubic Bones}

Character 83: Acetabulum morphology: (0) shallow, dorsal part of acetabular fossa not laterally expanded; (1) deep, with the dorsal part extended laterally (fig. 11). The acetabulum morphology is critical in femur flexion (Elftman, 1929). A shallow acetabular fossa allows a wide range of movements of the femur (Jenkins and Camazine, 1977; Argot, 2002), especially in abduction, which is related to arboreal habits (Elftman, 1929; Muizon and Argot, 2003). However, this morphology implies a reduced stability of the joint, because of as it was established for Caluromys (Muizon and Argot, 2003), arboreal didelphids pressumably have slow climbing habits. In this context, Metachirus is autapomorphic for this character since it exhibits a deep acetabular fossa, with the dorsal portion laterally extended, which is a specialized morphology for its cursosaltatorial mode of locomotion.

Character 84: Iliac wing forming a large blade: (0) absent; (1) present (fig. 11). The 


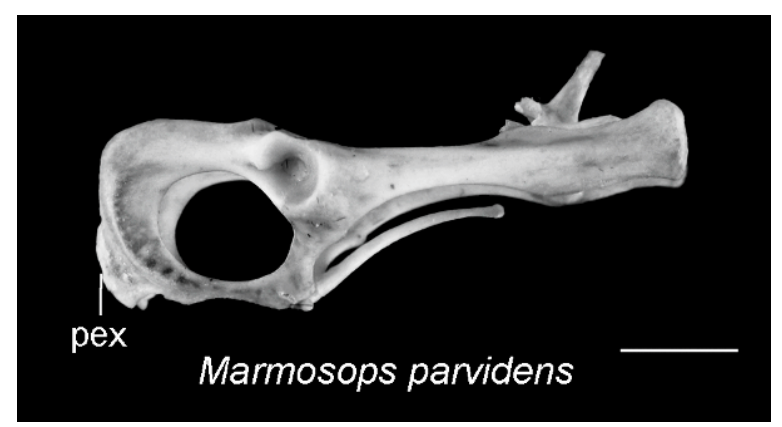

Fig. 23. Marmosops parvidens (AMNH 267348), os coxae in lateral view showing the posteroventral extension (pex) on the pubis (ch. 86[1]). Scale bar: $5 \mathrm{~mm}$.

iliac wing, which forms a large blade, is an autapomorphy only present in Metachirus, which shows a reduced iliac fossa as well. This extension is occupied by a well-developed m. gluteus medius (Argot, 2002; Taylor, 1974) and is indicative of high activity of this muscle as well as the development of the epaxial musculature (Maynard Smith and Savage, 1955; Grand, 1983). The morphology exhibited by Metachirus is consistent with the results of Grand (1983) as well, because the lower back musculature of this taxon represents more than $55 \%$ of the total epaxial musculature, different from the $25-35 \%$ in other didelphid taxa. Although the iliac fossa is also reduced in Metachirus due to the blade shape of the iliac wing, the $\mathrm{m}$. iliacus is well developed (Argot, 2002; Elftman, 1929).

Character 85: Angle formed by the two posterior rami of ischium in caudal view: (0) $90^{\circ}$ or scarcely more; (1) less than $90^{\circ}$. In caudal view, the angle formed by the two rami of ischium can accentuate the extroversion of the ischiatic spine, which is important in the origin of abductors and gracilis muscles (Elftman, 1929; Argot, 2002). Additionally, the angle has been related to the range of abduction (Jenkins and Camazine, 1977). As it was stated by Argot (2002), a sharp angle (or an oblique orientation of the ischium) emphasizes the degree of abduction. In the sample analyzed, the two rami of the ischium form an angle of $90^{\circ}$ in Metachirus, Chironectes, Philander, Didelphis, Lestodelphys, Hyladelphys, and Gracilinanus. In the remaining taxa, the angle is less than $90^{\circ}$.

Character 86: Presence of an osseous posteroventral extension on the ischium: (0) absent; (1) present (fig. 23). The posteroventral extension on the ischium is a synapomorphy observed only in the species of Marmosops. This extension seems to increase the area for origin of the hamstring muscles complex (i.e., Mm. biceps femoris, semimembranosus, and semitendinosus), which inserts on the tibia and fibula and contributes to the knee flexion.

Character 87: Development of iliopubic process: (0) absent; (1) present (fig. 24). This character was described by Horovitz and Sánchez-Villagra (2003: ch. 73). In this area the $\mathrm{m}$. psoas minor is inserted, which originates in the last thoracic and the first lumbar vertebrae, and whose action is to flex the lumbar part of the vertebral column (Elftman, 1929; Evans, 1993). Although in some specimens it was not be very conspicuous, the process is present in both terrestrial as well as arboreal forms, such as Caluromysiops, Metachirus, Lutreolina, and Thylamys pusillus. Polymorphism is evidenced in Philander frenatus and Chironectes (coded $\{01\}$ ).

Character 88: Epipubic bones, proximal size: (0) short; (1) long. In the same way as in Horovitz and Sánchez-Villagra (2003: ch. 77), I coded short proximal size of epipubic bones when the contact is equal to or less than half of the distance between the pubic symphysis and the point at the anterior edge of the pelvis, whose level is coincident with the middle of the acetabulum. The proximal size of the epipubic bones is long in Caluromysiops, Caluromys, Philander, Didelphius, Lutreolina, Chironectes, Thylamys, Monodelphis, Micoureus demerarae, Lestodelphys, Marmosa (except M. rubra), and Marmosops. 


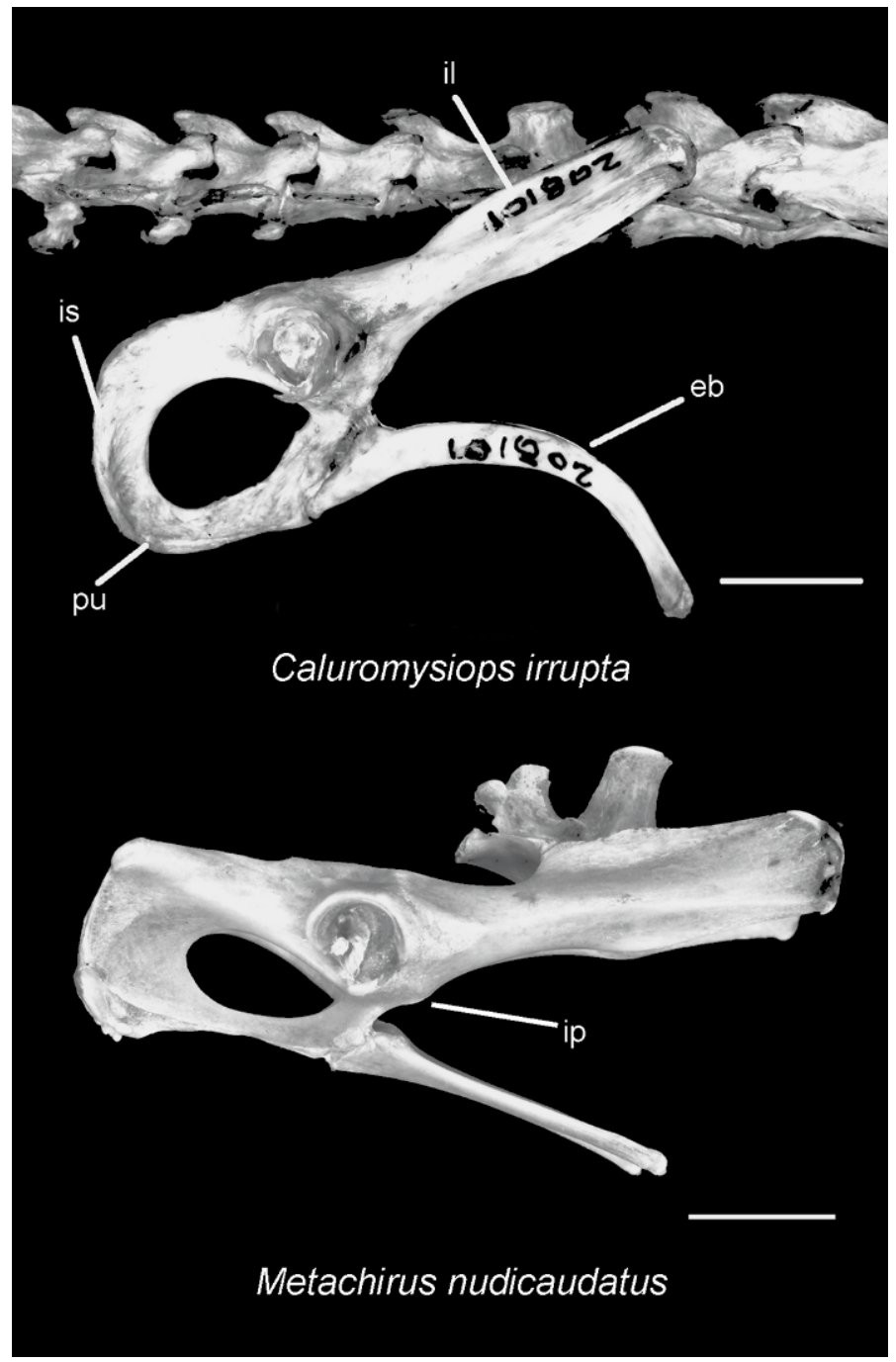

Fig. 24. Caluromysiops irrupta (AMNH 208101) and Metachirus nudicaudatus (AMNH 267009), right os coxae with epipubic bones (eb) in lateral view. The epipubic bones are notably curved (ch. 89[1]) in Caluromysiops, whereas in Metachirus the bones are straight (ch. 89[0]). Note the iliopubic (ip) process well developed in Metachirus (ch. 87[1]). Other abbreviations: il, ilium; is, ischium; pu, pubis. Scale bars: $10 \mathrm{~mm}$.

Polymorphism is exhibited only in Micoureus regina. No information about this character is currently available for Thylamys venustus and Philander mcilhennyi (coded “?").

Character 89: Epipubic bones distal shape: (0) straight; (1) curved (fig. 24). In general terms I founded evidence of sexual dimorphism in the development of epipubic bones (at least those taxa in which a good series was analyzed, see appendix 1), being larger in females of pouchless taxa (as observed by
White, 1989). Its shape shows two apparently defined morphotypes across the taxa analyzed. The condition of straight epipubic bones is the most common morphology exhibited in the sample. On the contrary, the epipubic bones are clearly curved in the distal portion in some taxa, such as Caluromysiops, Caluromys, Marmosops parvidens, Chironectes, and Marmosa rubra. No information is currently available about this character for Philander mcilhennyi (coded “?”). 


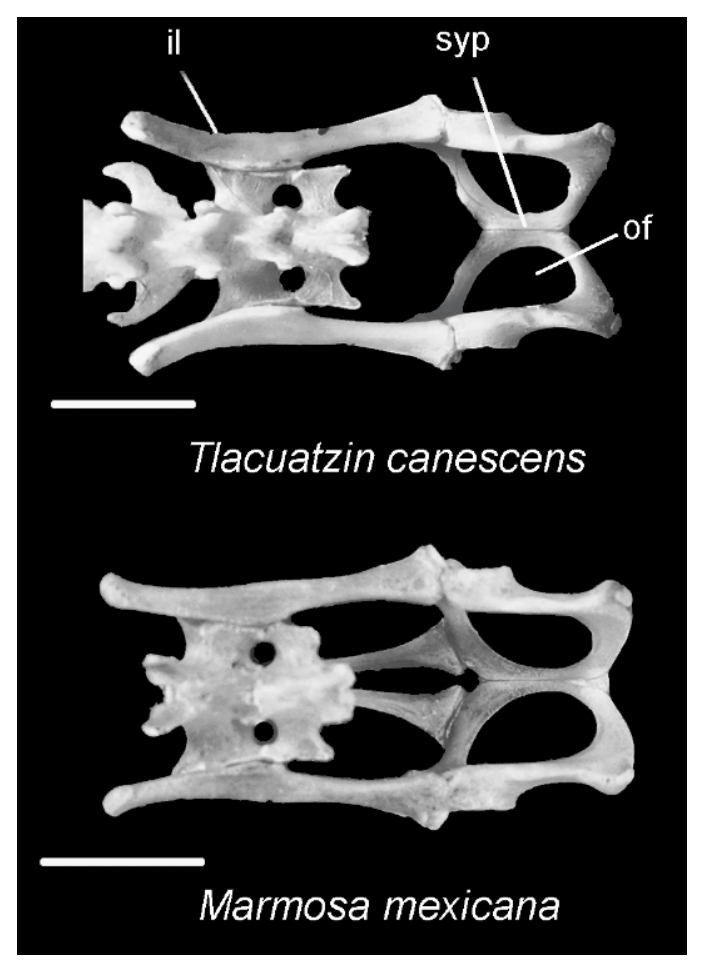

Fig. 25. Tlacuatzin canescens (UMMZ 94604) and Marmosa mexicana (ROM 99604), pelvis in dorsal view. In Tlacuatzin the symphysis pubis (syp) is shorter than the obturator foramen (of) (ch. 91[0]), whereas the symphysis size is similar to the obturator foramen in Marmosa mexicana (ch. 91[1]). Note the anterior portion of the ilium (il) curved laterally in Tlacuatzin (ch. 90[1]), and in Marmosa it is almost straight (ch. 90[0]). Scale bars: $5 \mathrm{~mm}$.

Character 90: Ilium shape: (0) straight; (1) with the distal portion barely curved laterally (fig. 25). In character 84, I considered the general development of the iliac wing. Here, I focused on the direction of the distal portion of the ilium. As mentioned above, the shape of the ilium is important in the movements of the hip, since this is the area of origin of some muscles involved in the extension-flexion of the hip joint such as the Mm. glutei, iliacus, and sartorius. The distal portion slightly curved laterally probably increased the development of the $\mathrm{m}$. glutei, as well as the epaxial musculature (Maynard Smith and Savage, 1956; Grand, 1983). Even though in the study of Argot (2002) the terrestrial Metachirus is the only didelphid species analyzed with this particular morphology of the ilium (see Argot, 2002: fig 7b), I found the same pattern in other didelphid species as well, such as Glironia, Chironectes, and Tlacuatzin, which are not always directly associated with terrestrial habits.

Character 91: Pubic symphysis size in relation to the craniocaudal size of the obturator foramen: (0) shorter; (1) equal or longer (fig. 25). Although the obturator foramen is well developed in all didelphid species analyzed herein, I detected a remarkable variation in pubic symphysis pubis size. Variation in this character could be linked with the area of origin of $\mathrm{m}$. gracilis, implied in the flexion of the tibia (Elftman, 1929), and assisting the hamstring muscles in the extension of the femur (Argot, 2002; Maynard Smith and Savage, 1955). In most didelphid species, the symphysis is equal to or longer than the obturator foramen. However, in Glironia, Tlacuatzin, and Thylamys macrurus, the symphysis is shorther than the obturator foramen.

Character 92: Caudal portion of ischium body curved laterally (other than the ischiatic tuberosity): (0) absent; (1) present (fig. 26). This is the region of the ischium where important muscles involved in the flexion of the tibia and tail movements originate, such as $\mathrm{Mm}$. biceps femori and semitendinosus. The curved shape of the ischium probably increases the function of those muscles. Both muscles (i.e., Mm. biceps femoris and semitendinosus) are well developed in Metachirus, probably due to the typical cursosaltatorial locomotion (Maynard Smith and Savage, 1956). Besides Metachirus, I found the posterior part of ischium body to be laterally curved also in Philander, Didelphis, Lutreolina, and Chironectes.

\section{FEMUR}

Character 93: Development of lesser trochanter: (0) not very developed; (1) well developed, surpassing the half of the mesial extension of the femoral head (fig. 27). Although the lesser trochanter is present in all didelphids, I detected two character states for its development. In this structure the Mm. iliacus and psoas major insert, which is implied in the flexion of the hip joint, as well 


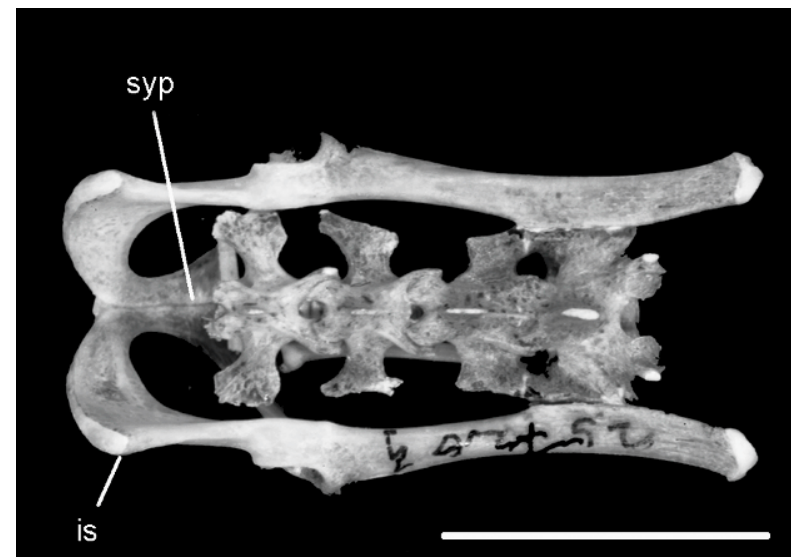

\section{Monodelphis brevicaudata}

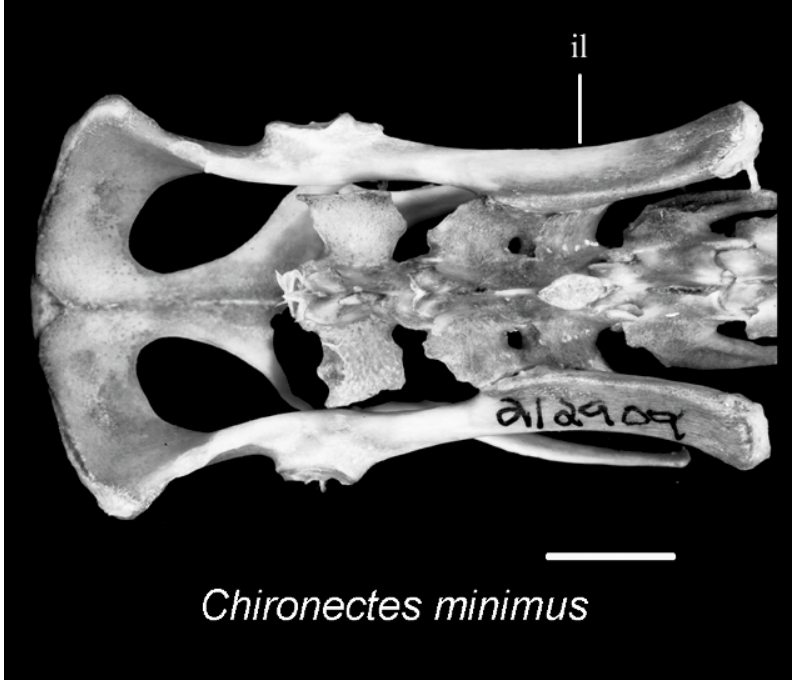

Fig. 26. Monodelphis brevicaudata (AMNH 257203) and Chironectes minimus (AMNH 212909), pelvis in dorsal view. In Chironectes the posterior part of the ischium (is) body is laterally deflected (ch. 92[1]), whereas in Monodelphis it is almost straight (ch. 92[0]). Other abbreviations: il, illium; syp, symphysis pubis. Scale bars: $10 \mathrm{~mm}$.

as the flexion and fixation of the vertebral column (Evans, 1993). In arboreal forms, such as Caluromys or Micoureus, the iliacus and psoas major act as flexors and as external rotators and adductor of the leg (Muizon and Argot, 2003). Argot (2002) reported differences on the development of the lesser trochanter of Caluromys, Metachirus, Monodelphis, and Micoureus. In the sample analyzed, the lesser trochanter is notably developed in Glironia, Caluromys, Caluromysiops, Thylamys pusillus, T. macrurus, Micoureus, Marmosa (except M. rubra), Marmosops,
Cryptonanus, and Gracilinanus. No information is currently available about this character for Thylamys venustus (coded '?"').

Character 94: Protuberance between trochanteric fossa and head: (0) absent; (1) present (fig. 27). This protuberance seems to be an impression of the insertion of obturatores and gemelli muscles. It is present in most species analyzed herein, except for $T$. pallidior, which is autapomorphic in this character, as this structure is absent. No information is currently available about this character for Thylamys venustus (coded "'?"). 


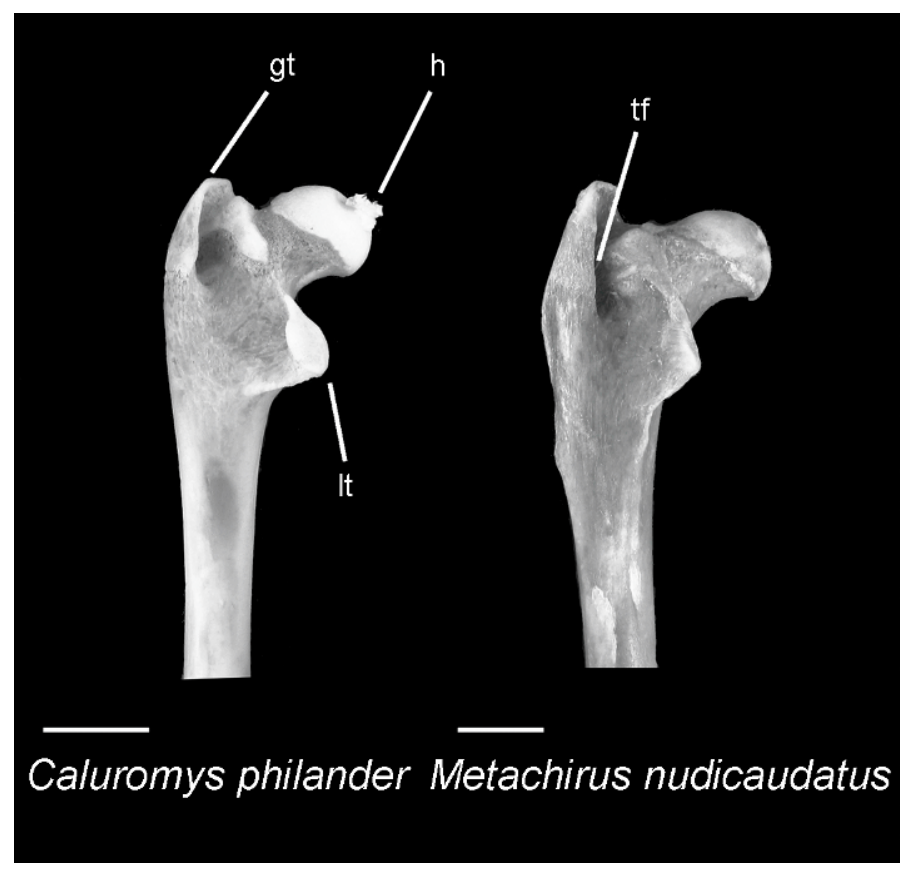

Fig. 27. Caluromys philander (AMNH 267001) and Metachirus nudicaudatus (AMNH 244617), proximal portion of right femur. In Metachirus, the greater trochanter (gt) is well developed (ch. 95[1]), whereas in Caluromys it is not very developed (ch. 95[0]). The lesser trochanter (1t) is more developed in Caluromys (ch. 93[1]) than in Metachirus (ch. 93[0]). Note in both species the protuberance between the trochanteric fossa (tf) and femoral head (h) (ch. 94[1]). Scale bars: $5 \mathrm{~mm}$.

Character 95: Development of greater trochanter: (0) not surpassing the level of the head; (1) surpassing the level of the head (fig. 27). According to Argot (2002), didelphids usually exhibit a greater trochanter not higher than the femoral head. However, besides Metachirus, I judged greater trochanter notably developed in Didelphis, Philander, Lutreolina, Chironectes, Tlacuatzin, Marmosa robinsoni, M. mexicana, Marmosops impavidus, M. parvidens, and M. pinheiroi.

Character 96: Distal epiphysis anteroposteriorly compressed: (0) absent; (1) present. The anteroposterior compression of the distal femoral epiphysis is probably related to the intercondyloid fossa being delimited by low crests (Argot, 2002). Some didelphids with well-developed arboreal habits (Caluromys, Micoureus) show this morphology (Muizon and Argot, 2003), and the same relationship was evidenced in viverrids (Taylor, 1976) and primates (Tardieu, 1983). In the sample, the distal epiphysis is anteroposteriorly compressed in Caluromys, Caluromysiops, Philan- der, Marmosa mexicana, M. murina, $M$. rubra, and Micoureus.

\section{Tibia AND FibUlA}

Character 97: Sesamoids in the articular area between tibia, fibula, and astragalus: (0) absent; (1) one sesamoid present. This character is modified from Horovitz and SánchezVillagra (2003: ch. 86), since in the sample there are no taxa with two sesamoids. One sesamoid in the area between tibia, fibula, and astragalus is present in Glironia, Metachirus, Philander, Didelphis virginiana, Tlacuatzin, Thylamys, Monodelphis, Marmosops incanus, M. parvidens, M. pinheiroi, and Cryptonanus unduaviensis. Polymorphism is exhibited only in Chironectes (coded $\{01\}$ ). No information is currently available about this character for Philander mcilhennyi, Marmosa rubra, Lutreolina, Thylamys pusillus, Lestodelphys, and Gracilinanus microtarsus (coded “?”).

Character 98: Tibia length relative to femur length: (0) tibia shorter than femur; 


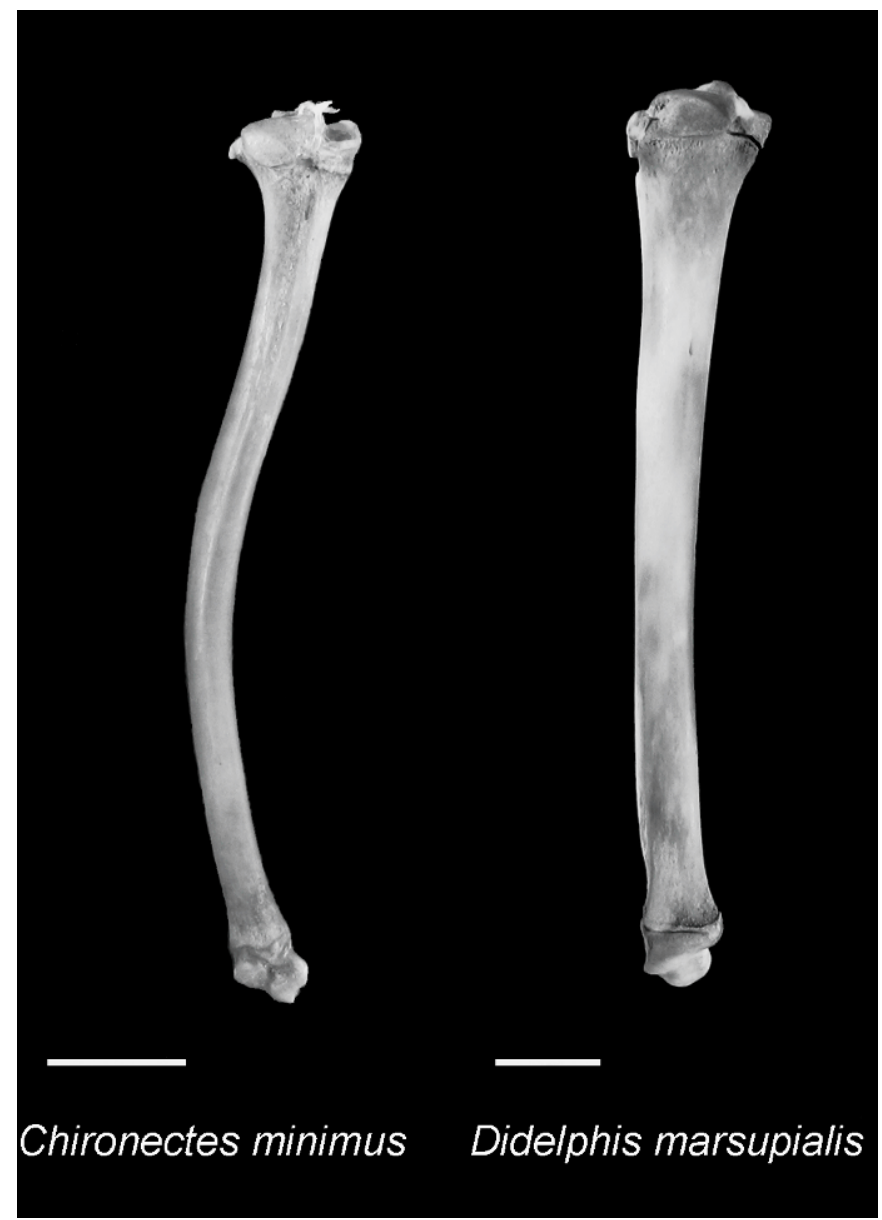

Fig. 28. Chironectes minimus (AMNH 212909) and Didelphis marsupialis (AMNH 210439), right tibia in anterior view. In Chironectes the tibia has a sigmoid shape (ch. 99[0]), whereas in Didelphis it is almost straight (ch. 99[1]). Scale bars: $5 \mathrm{~mm}$.

(1) tibia longer than or equal to femur. This character was described by Horovitz and Sánchez-Villagra (2003: ch. 89). Glironia is autapomorphic in this character, as the femur is longer than the tibia. Maynard Smith and Savage (1955) found similar proportions only in large mammals, such as Rhinoceros, Mastodon, and horses. In marsupials, this characteristic was also evidenced in some Australasian taxa with a diversity of habits, such as Phalanger, Pseudochirops, Phascolarctos, and Vombatus (Horovitz and Sánchez-Villagra, 2003), as well as the fossil Mayulestes (Muizon, 1998). In the remaining American marsupials, the tibia is longer than the femur (see table 3 in Hershkovitz, 1999).
Character 99: Tibia shape: (0) sigmoidshaped; (1) sigmoid shape present but not so marked (fig. 28). Although the sigmoid shape of the tibia is the most common condition in didelphids, some taxa exhibit a notable sigmoid shape. This morphology was interpreted as plesiomorphic and is not restricted to didelphids (Szalay and Sargis, 2001). The diaphysis starts in a sigmoid curvature at the level of the insertion of hamstring muscles, which suggests the possibility that this shape appears due to the pull of these extensors of the leg (Argot, 2002). On the other hand, Lanyon (1980) demonstrated the biomechanical advantages of the curved shape on load transmission. The sigmoid shape of the tibia 


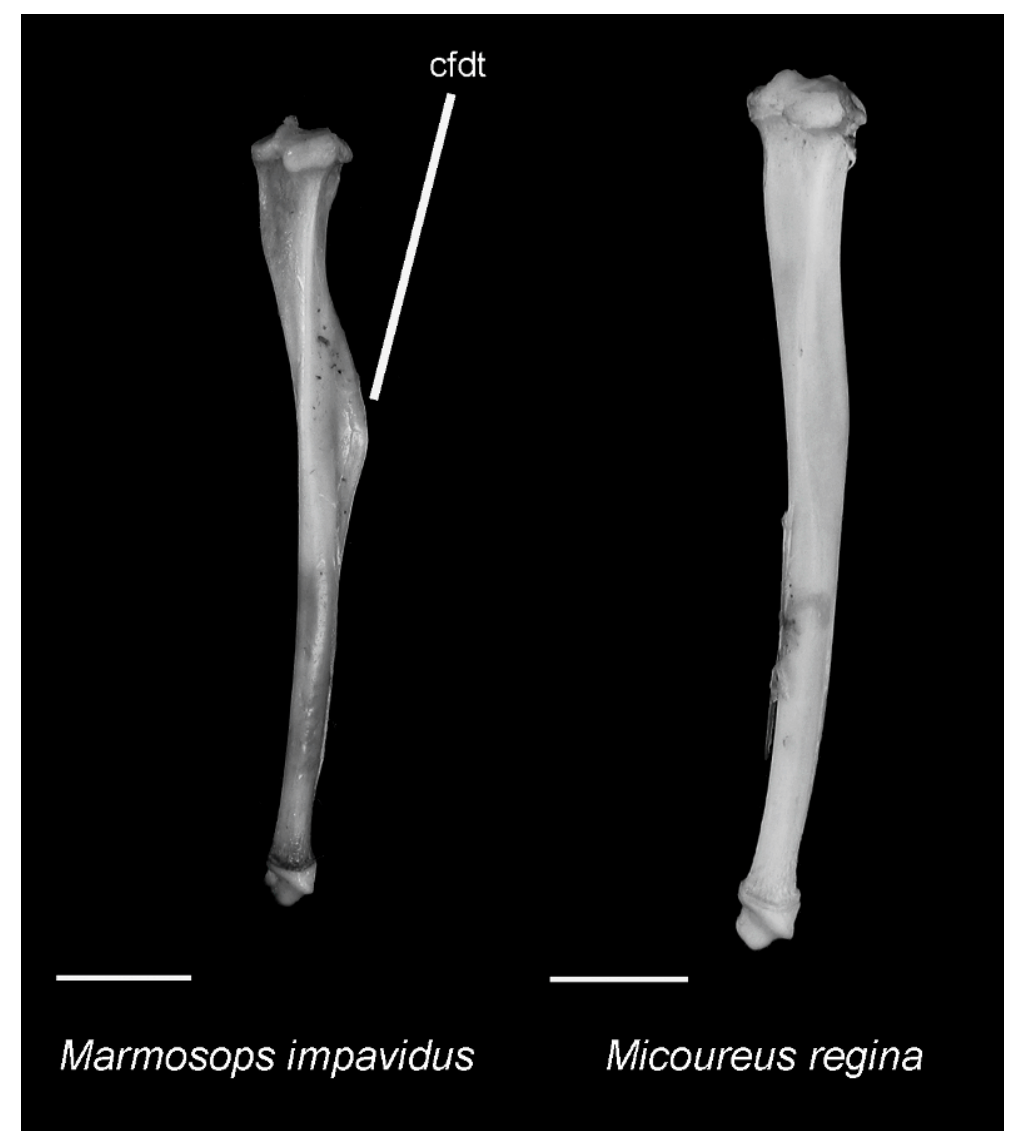

Fig. 29. Marmosops impavidus (AMNH 139226) and Micoureus regina (AMNH 148757), right tibia in mesial view. In Marmosops there is a notable crest for insertion of $\mathrm{m}$. flexor digitorum tibialis (cfdt) (ch. 101[1]), which is absent in Micoureus (ch. 101[0]). Scale bars: $5 \mathrm{~mm}$.

is also related to the asymmetrical condition of the femoral condyles in the knee joint. The lateral displacement of the load line is linked to the tibia shape and its function on load transmission (Szalay and Sargis, 2001). In the sample, Chironectes, Tlacuatzin, and Gracilinanus microtarsus exhibit a remarkably sigmoid-shaped tibia.

Character 100: Tibial tuberosity developed: (0) absent; (1) present. The tendon of the $\mathrm{m}$. quadriceps, a powerful extensor of the knee, inserts directly on the tibial tuberosity, as the patella is absent in didelphids. In some taxa with well-developed arboreal habits (e.g., Caluromys, Caluromysiops, Micoureus), the tibial tuberosity is neither very evident or anteriorly expanded (personal obs.; Muizon and Argot, 2003). Only the terrestrial $\mathrm{Me}$ tachirus exhibits this structure as notably developed, which is coherent with a more stabilized knee joint, useful for the saltatorial mode of locomotion. In slow-climbing didelphids, a more mobile and less stabilized knee joint is necessary, because of the range of hindlimb movements during arboreal displacement.

Character 101: Tibia, development of the posterior crest for the insertion of $\mathrm{m}$. flexor digitorum tibialis: (0) absent; (1) present (fig. 29). The crest is present in Hyladelphys, Marmosa mexicana, Marmosops noctivagus, M. impavidus, M. incanus, Lestodelphys, Gracilinanus, and Cryptonanus.

Character 102: Head of fibula notably developed craniocaudally: (0) absent; (1) present. An anteroposteriorly expanded head of the fibula is associated with the development of the area of insertion of the $\mathrm{m}$. 


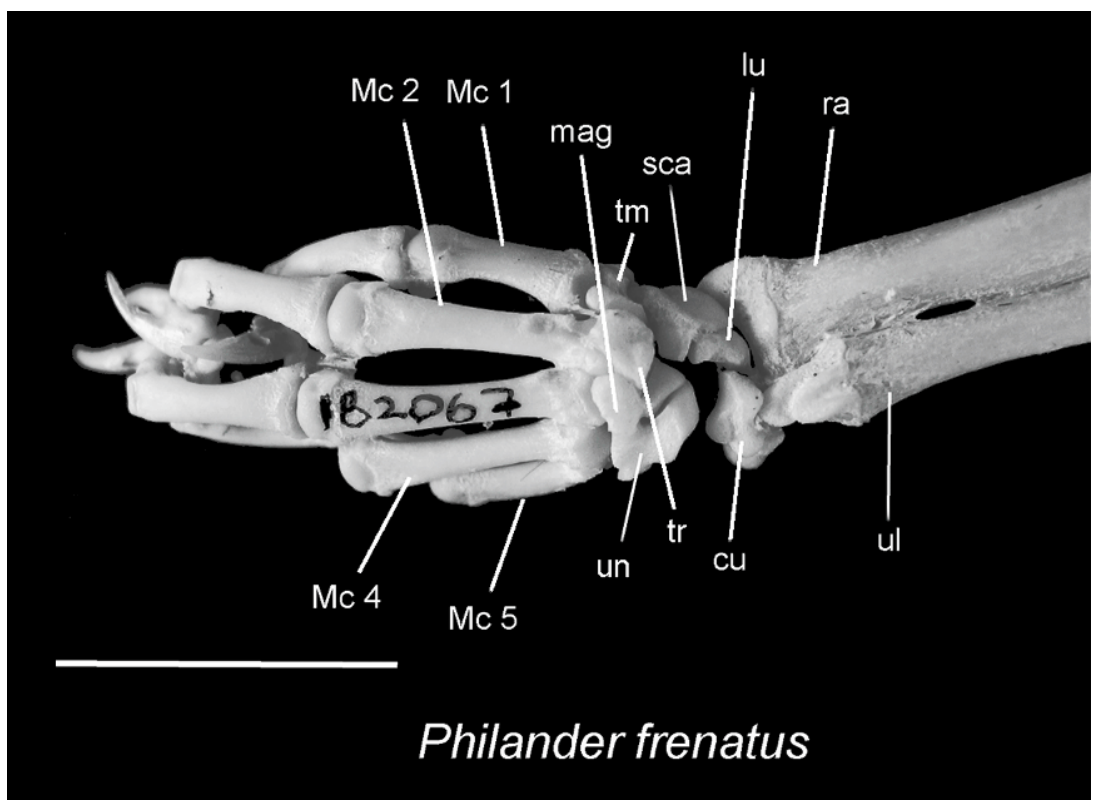

Fig. 30. Philander frenatus (MVZ 182067), dorsal aspect of distal ulna (ul), radius (ra), carpal, and metacarpal (Mc) bones. Note the lunate (lu) relatively large and in contact with other elements (ch. 103[1]). A distolateral process of the scaphoid (sca) separates the lunate and magnum (mag) (ch. 105[1]). Other abbreviations: un, unciform; cu, cuneiform; tm, trapezium; tr, trapezoid. Scale bar: $5 \mathrm{~mm}$.

peroneus longus, which is involved in flexion of the tarsus (Evans, 1993). Consequently, this is linked to arboreal habits, as the mentioned muscle inserts on the proximal portion of the first metatarsal (Muizon and Argot, 2003; Argot, 2003a) and is associated with the opposability of the hallux. The head of the fibula is notably developed anteroposteriorly in Caluromys, Caluromysiops, Chironectes, Tlacuatzin, Marmosa, Marmosops, Thylamys (except T. macrurus), Lestodelphys, Micoureus, Gracilinanus, and Cryptonanus.

\section{Carpus And Metacarpus}

Character 103: Lunate: (0) small (contacting only with scaphoid and cuneiform); (1) relatively large (contacting with scaphoid, cuneiform, magnum, and unciform) (fig. 30). This character is modified from Horovitz and Sánchez-Villagra (2003: ch. 62) since in this sample there are no taxa with the lunate absent or fused with other elements. In most didelphids, the lunate is relatively large and in contact with other elements. However, this bone is notably smaller in Philander mcilhen- nyi, Tlacuatzin, Thylamys pusillus, Marmosa murina, Marmosops, Cryptonanus unduaviensis, and Gracilinanus agilis. No information is currently available about this character for Micoureus regina, M. paraguayanus, Lestodelphys, and Gracilinanus microtarsus (coded "?").

Character 104: Prepollex: (0) absent; (1) present. This character was described by Horovitz and Sánchez-Villagra (2003: ch. 63). Because the prepollex is the smallest element on the wrist, its loss is common during the cleaning process. The prepollex is present in most groups analyzed herein, except for Metachirus, Chironectes, and Thylamys pusillus, where this element seems to be absent. No information is currently available about this character for Lutreolina, Tlacuatzin, Micoureus paraguayanus, Lestodelphys, Marmosops impavidus, M. noctivagus, Cryptonanus unduaviensis, and Gracilinanus microtarsus (coded “?”).

Character 105: Distolateral process of scaphoid separating lunate from magnum dorsally: (0) absent; (1) present (fig. 30). This character was described by Horovitz and 
Sánchez-Villagra (2003: ch. 64). This process of the scaphoid is present in most groups analyzed, except for Glironia and Cryptonanus unduaviensis. No information is currently available about this character for Lutreolina, Tlacuatzin, Monodelphis brevicaudata, $M$. adusta, Micoureus paraguayanus, Lestodelphys, and Gracilinanus microtarsus (coded "?").

\section{TARSUS}

Character 106: Astragalus, angle between medial and lateral facets for tibia: (0) intermediate, between $90^{\circ}$ and $180^{\circ}$; (1) $180^{\circ}$. This character is modified from Horovitz and Sánchez-Villagra (2003: ch. 94), since in the current sample there are no taxa with a $90^{\circ}$ angle, and the character is treated as binary. According to Jenkins and McClearn (1984) and Szalay (1982, 1994), the medial and lateral astragalotibial facets form a broad and almost flat plane in didelphids. However, I observed a continuous variation in the angle formed by the facets, and all conditions were met in a single interval $\left(90^{\circ}-180^{\circ}\right)$. In terrestrial forms, as Metachirus or Monodelphis, the facets are better delimited, forming a sharper angle (personal obs.; Szalay, 1994). On the other hand, the angle between medial and lateral facets for the tibia was $180^{\circ}$ only in some large opossums such as Philander, Lutreolina, Didelphis, and Chironectes. No information is currently available about this character for Monodelphis adusta, Micoureus paraguayanus, and Lestodelphys (coded '?"').

Character 107: Astragalus, dimensions of astragalonavicular facet in distal view: (0) transversely wider; (1) dorsoventrally wider. This character was described by Horovitz and Sánchez-Villagra (2003: ch. 97). In the taxa analyzed here, only Metachirus exhibits the astragalonavicular facet dorsoventrally wider. No information is currently available about this character for Monodelphis adusta, Micoureus paraguayanus, and Lestodelphys (coded “?").

Character 108: Astragalus, ridge between medial and lateral astragalotibial facets: (0) absent; (1) present (fig. 31). This character was described by Horovitz and SánchezVillagra (2003: ch. 107). The ridge between

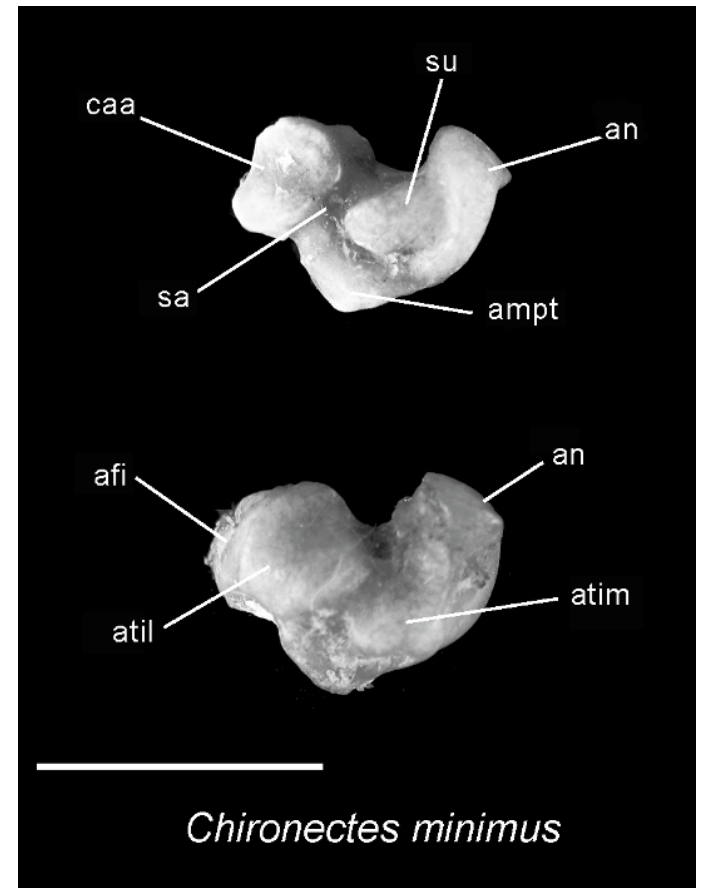

Fig. 31. Chironectes minimus (AMNH 148720), plantar and dorsal views of left astragalus. The ridge between the medial (atim) and lateral (atil) astragalotibial facets is present (ch. 108[1]), as well as being between the lateral atragalotibial and astragalofibular (afi) facets (ch. 109[1]). There is no contact between the astragalonavicular (an) and sustentacular (su) facets (ch. 110[0]), and the sustentacular facet is separated from the calcaneoastragalar (caa) facet by the well-developed sulcus astragali (sa) (ch. 111[0]). Other abbreviation: ampt, astragalar medial plantar tuberosity. Scale bar: $5 \mathrm{~mm}$.

medial and lateral astragalotibial facets is present in large opossums, such as Glironia, Caluromys, Caluromysiops, Didelphis, Metachirus, Lutreolina, and Chironectes. Contrary to Horovitz and Sánchez-Villagra (2003), I coded 0 for Monodelphis, since I did not observe a ridge in this area of the astragalus. No information is currently available about this character for Monodelphis adusta, Micoureus paraguayanus, and Lestodelphys (coded “?”).

Character 109: Astragalus, ridge between lateral astragalotibial facet and astragalofibular facet: (0) absent; (1) present (fig. 31). This character was described by Horovitz and Sánchez-Villagra (2003: ch. 108). Similar 
to the anterior character, the ridge is present only in large opossums. No information is currently available about this character for Monodelphis adusta, Micoureus paraguayanus, and Lestodelphys (coded '"?").

Character 110: Astragalus, contact between astragalonavicular and sustentacular facets: (0) absent; (1) present. This character was described by Horovitz and SánchezVillagra (2003: ch. 110). I found evidence of contact between both facets in most taxa, except Glironia, Caluromysiops, Metachirus, Chironectes (fig. 31), and Thylamys pallidior. Individual variation is observed in Didelphis virginiana and D. marsupialis (coded $\{01\}$ ). No information is currently available about this character for Monodelphis adusta, Micoureus paraguayanus, and Lestodelphys (coded "?").

Character 111: Astragalus, continuous lower ankle joint pattern: (0) absent (fig. 31); (1) present. This pattern results from the contact between posterior calcaneoastragalar and sustentacular facets, which is in relation to the absence of the sulcus astragali. I found evidence of the absence of this pattern in most didelphid groups analyzed here, except for Gracilinanus microtarsus and Hyladelphys, where a continuous lower ankle joint pattern is observed. Opposite to this, individual variation is observed in Marmosa mexicana. No information is currently available about this character for Monodelphis adusta, Micoureus paraguayanus, and Lestodelphys (coded "'?").

Character 112: Astragalus, astragalonavicular facet vertically oriented and distal calcaneocuboid facet deep: (0) absent; (1) present. Most didelphid groups analyzed herein exhibit the astragalonavicular facet transversely oriented, except for the terrestrial Metachirus, where the astragalonavicular facet is almost vertically oriented, which is accompanied by an increase in depth of the distal calcaneocuboid facet of the calcaneus. Although the orientation of the astragalonavicular facet of Monodelphis seems to be somewhat vertical (as was also observed by Szalay, 1994: 191), its position reflects the condition exhibited by most of didelphids groups (i.e., condition 0). The particular vertical orientation of the astragalonavicular facet showed by Metachirus (and partially by

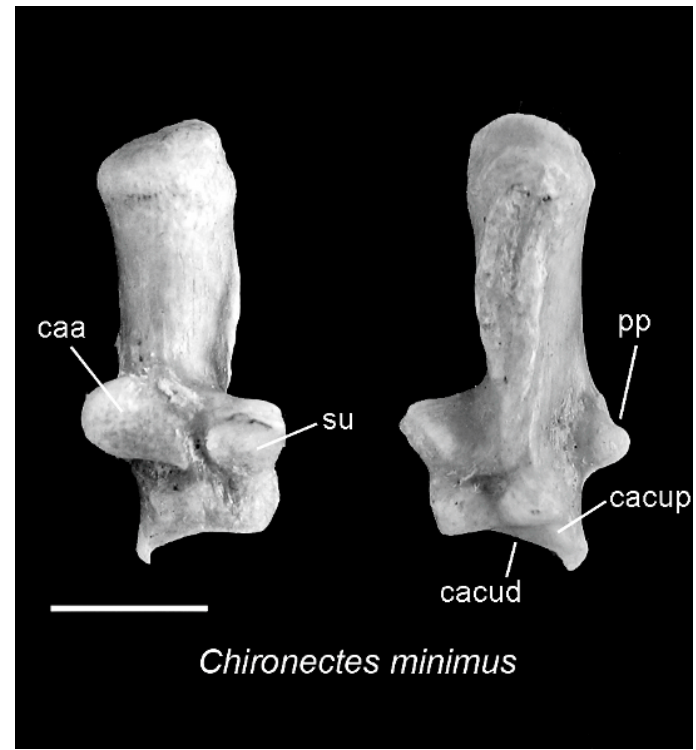

Fig. 32. Chironectes minimus (AMNH 148720), dorsal and plantar views of right calcaneus. The peroneal process (pp) is well developed (ch. 113[1]), and the calcaneal sustentaculum (su) is placed on the anterior tip (ch. 114[1]). Other abbreviations: caa, calcaneoastragalar facet; cacud, distal calcaneocuboid facet; cacup, proximal calcaneocuboid facet; sc, sulcus calcanei. Scale bar: $5 \mathrm{~mm}$.

Monodelphis) suggests the increased functional importance of flexion-extension rather pronation-supination of the hindfoot. Polymorphism is evidenced in Philander opossum (coded $\{01\}$ ). No information is currently available about this character for Monodelphis adusta, Micoureus paraguayanus, and Lestodelphys (coded “'?').

Character 113: Calcaneus, development of peroneal process of calcaneus: (0) small; (1) well developed (fig. 32). Most of the didelphid group analyzed herein exhibits the peroneal process well developed, except for Metachirus and Monodelphis, where this process is smaller (see Szalay, 1994: figs. 812). No information is currently available about this character for Monodelphis adusta, Micoureus paraguayanus, and Lestodelphys (coded “'?").

Character 114: Calacaneus, calcaneal sustentaculum position: (0) subterminal; (1) on anterior tip (fig. 32). The calcaneal sustentaculum is placed on the anterior tip of the calcaneum only in Chironectes. No informa- 
TABLE 5

Tree Statistics from Parsimony Analyses of Different Didelphid Data Sets, Considering Polymorphism as Composite Entries (CO) and Transformation Series (TS)

\begin{tabular}{|c|c|c|c|c|c|c|c|c|c|c|}
\hline & \multicolumn{2}{|c|}{$\begin{array}{l}\text { Morphology (Jansa } \\
\text { and Voss, 2005) } \\
\end{array}$} & \multicolumn{2}{|c|}{ Postcranial } & \multicolumn{2}{|c|}{$\begin{array}{c}\text { Nonmolecular } \\
\text { data set combined }^{\mathrm{b}}\end{array}$} & \multicolumn{2}{|c|}{ Total evidence ${ }^{c}$} & \multicolumn{2}{|c|}{ Total evidence ${ }^{\mathrm{d}}$} \\
\hline & $\mathrm{CO}$ & TS & $\mathrm{CO}$ & TS & $\mathrm{CO}$ & TS & $\mathrm{CO}$ & TS & $\mathrm{CO}$ & TS \\
\hline No. MPTs ${ }^{\mathrm{e}}$ & 76 & 1098 & 3 & 7 & 120 & 6 & 2 & 4 & 6 & 4 \\
\hline Consistency index $(\mathrm{CI})^{\mathrm{f}}$ & f $\quad 0.50$ & 0.50 & 0.27 & 0.25 & 0.33 & 0.29 & 0.61 & 0.59 & 0.46 & 0.43 \\
\hline Retention index (RI) & 0.84 & 0.84 & 0.62 & 0.59 & 0.68 & 0.65 & 0.80 & 0.79 & 0.75 & 0.72 \\
\hline Tree length $^{\mathrm{g}}$ & 178 & 177 & 502 & 540 & 723 & 926 & 6203 & 6464 & 1938 & 2192 \\
\hline Resolved ingroup nodes & 20 & 28 & 31 & 18 & 29 & 27 & 38 & 31 & 36 & 37 \\
\hline Nodes with $\geq 4$ absolute & 5 & 7 & 4 & 6 & 9 & 10 & 26 & 20 & 23 & 20 \\
\hline Bremer support & $(25 \%)$ & $(25 \%)$ & $(13 \%)$ & $(33 \%)$ & $(31 \%)$ & $(37 \%)$ & $(68 \%)$ & $(64 \%)$ & $(64 \%)$ & $(54 \%)$ \\
\hline
\end{tabular}

${ }^{a}$ Nonmolecular data set including craniodental, external morphology, and karyotype; defined by Voss and Jansa (2003).

${ }^{b}$ Morphological data set combining 71 nonmolecular characters defined by Voss and Jansa (2003), and 114 postcranial characters described in this report.

${ }^{\mathrm{c}}$ All combined evidence including IRBP, DMP-1, RAG-1, and morphology.

${ }^{\mathrm{d}}$ Partitioned combined evidence including IRBP, DMP-1, and morphology (RAG-1 eliminated).

eEqually most parsimonious trees recovered by heuristic searches as described in "Materials and Methods".

${ }^{\mathrm{f}}$ Excluding autapomorphies.

${ }^{\mathrm{g}}$ Including autapomorphies.

tion is currently available about this character for Monodelphis adusta, Micoureus paraguayanus, and Lestodelphys (coded “?”).

\section{Postcranial Data Set Summary}

The data set described above includes 114 postcranial characters, of which $106(93 \%)$ are parsimony informative and $8(7 \%)$ are autapomorphic. Sixty-six characters $(58 \%)$ are binary, $47(41 \%)$ describe ordered multistate (additive) transformations, and only 1 character $(0.9 \%)$ describes unordered multistate (nonadditive) transformations (table 3 ). The data matrix (appendix 2) has $114 \times 38=4332$ cells, of which only $79(2 \%)$ are scored as missing (“?") and $44(1 \%)$ are scored as inapplicable ("-"). The remaining 4209 matrix cells $(97 \%)$ record organismal traits, with data completeness for individual terminal taxa ranging from 88 to $100 \%$ (table 4 ). Polymorphism was detected for 30 characters $(2 \%$ of the total matrix cells), which showed intraspecific variation in the sample analyzed (table 2 ).

\section{Analytic Results of POSTCRANIAL CHARACTERS}

A heuristic analysis of the postcranial data analyzed with $\mathrm{CO}$ polymorphic entries re- sulted in three equally most parsimonious trees (502 steps, CI $=0.27, \mathrm{RI}=0.62$; table 5) whose highly resolved strict consensus is shown in figure 33 . The three caluromyines included (except the root) form a single clade in which Caluromys is recovered as a monophyletic group. Unlike results from the previous analysis of a different nonmolecular data set analyzed by Jansa and Voss (2005: fig. 1C), the deep branch topology in the didelphine group is well resolved in the consensus topology, and some already recognized groups are recovered. Hyladelphys appears in basal and intermediate positions between caluromyines and didelphines, which is consistent with hypotheses attained by previous nonmolecular data, IRBP sequences, and combined analyses (table 6). Successively, Marmosa robinsoni and Cryptonanus unduaviensis appear as sister taxa of the remaining didelphines, which form a wellresolved topology. As in previous morphological and molecular analyses, Thylamys is recovered as a monophyletic group, arranged in the sequence ( $T$. pallidior- $T$. venustus ( $T$. macrurus ( $T$. pusillus))). On the other hand, all species of Marmosops included in this analysis (pinheiroi, parvidens, noctivagus, impavidus, and incanus) form a monophyletic group (node $\mathrm{M}$ ), which have been recovered 


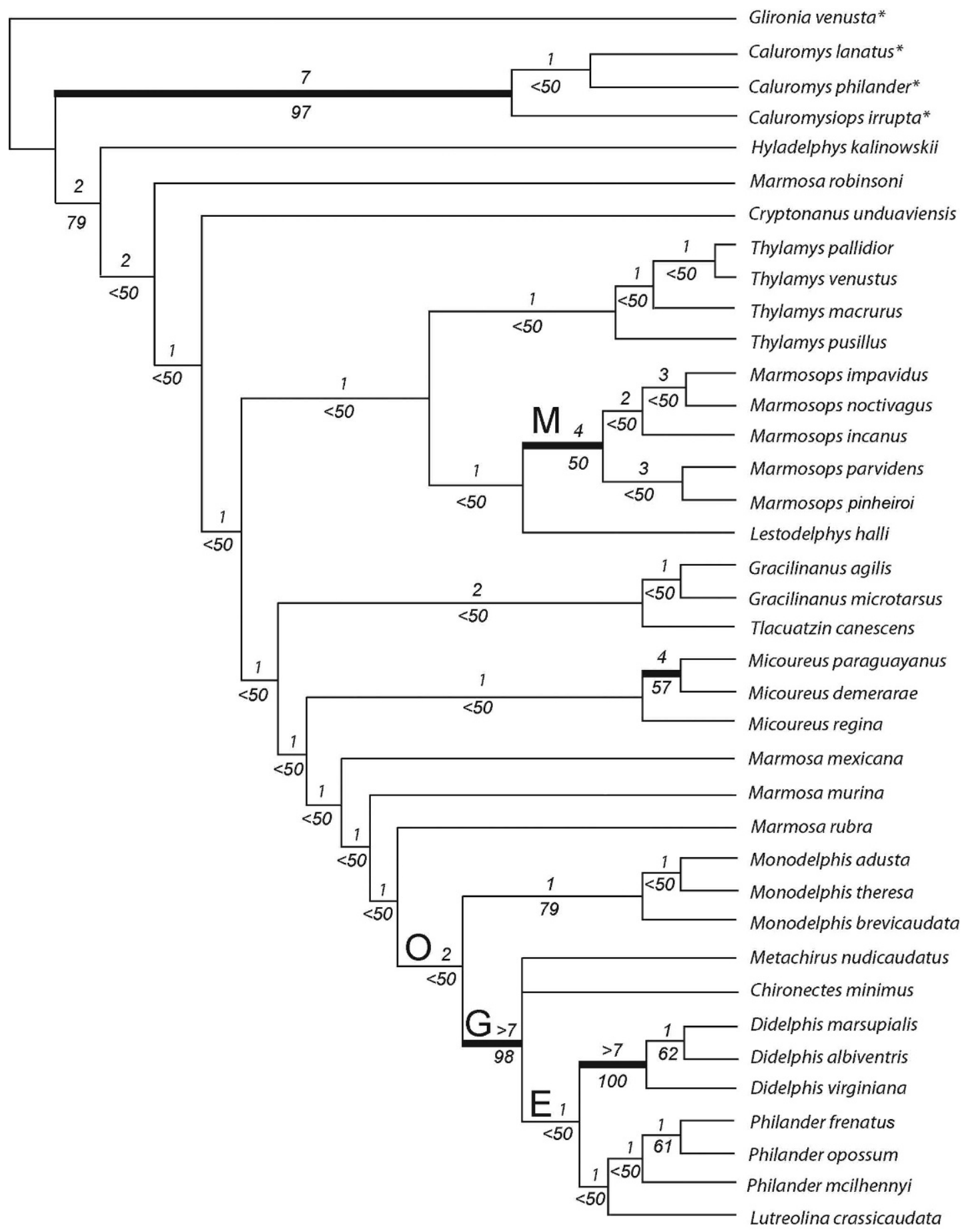

Fig. 33. Strict consensus of three equally most parsimonious trees resulting from cladistic parsimony analysis of 114 postcranial characters for 38 taxa described in this report, where polymorphic data were treated as composite entries (CO) (see tables 2-4 for summary data set characteristics and tree statistics). Numbers above branches refer to absolute Bremer support values $(\geq 1)$. Numbers below branches refer to 
TABLE 6

Different Data Sets Supporting an Intermediate Position of Hyladephys between Caluromyinae and Didelphinae.

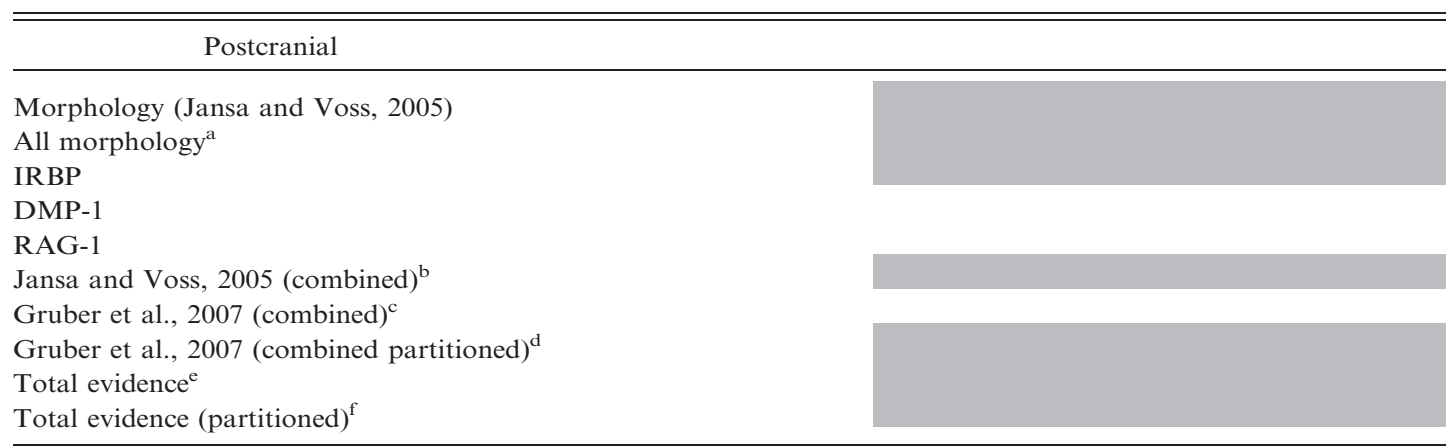

\footnotetext{
${ }^{a}$ Morphological data set including postcranial evidence described here, and the nonmolecular characters defined by Voss and Jansa (2003).

${ }^{\mathrm{b}}$ Data set combining nonmolecular characters, IRBP, and DMP-1 sequences (postcranial excluded).

${ }^{c}$ Data set combining nonmolecular characters, IRBP, DMP-1, and RAG-1 sequences (postcranial excluded).

${ }^{\mathrm{d}}$ Data set combining nonmolecular characters, IRBP, DMP-1, and RAG-1 sequences (postcranial excluded, RAG-1 third position eliminated).

${ }^{\text {e}}$ Data set combining nonmolecular characters, IRBP, DMP-1, and RAG-1 sequences (postcranial included).

${ }^{\mathrm{f}}$ Data set combining nonmolecular characters, IRBP, and DMP-1 (postcranial included, RAG-1 excluded).
}

in previous results with other kinds of evidence. In this group, Marmosops impavi$d u$ and $M$. noctivagus are sister species, as well as $M$. parvidens and M. pinheiroi. Lastly, Lestodelphys is placed as sister of Marmosops. Up in the tree, Tlacuatzin appears as sister of the group Gracilinanus agilis-G. microtarsus, and Micoureus is recovered as a monophyletic group in the sequence $(M$. demerarae-M. paraguayanus) $M$. regina)). The three remaining species of marmosa (M. mexicana, M. murina, and $M$. rubra) are successively arranged. Finally, the species of Monodelphis, resolved in the sequence ( $M$. brevicaudata ( $M$. adusta $+M$. theresa)) are recovered as sister group of the large opossums (node O). Although in general the large opossums (Didelphis, Philander, Metachirus, Chironectes, and Lutreolina; node G) form an unresolved clade, Didelphis is recovered as a monophyletic group in the sequence (Didelphis virginiana (D. marsupialis $+D$. albiventris), as well as Philander in the sequence ( $P$. mcilhennyi ( $P$. opossum-P. fre- natus)). Both genera form a monophyletic group with Lutreolina (node E).

The heuristic search of the postcranial data set with polymorphic entries analyzed with TS resulted in seven most parsimonious trees of 540 steps $(\mathrm{CI}=0.25, \mathrm{RI}=0.59)$. The strict consensus (fig. 34) resulted in a less resolved topology than $\mathrm{CO}$ analysis, where Hyladelphys appears again in an intermediate position. Under this criterion, the species of Marmosa, Gracilinanus, Cryptonanus unduaviensis, and Tlacuatzin are arranged in a basal polytomy. The monophyletic Monodelphis is resolved in the sequence ( $M$. adusta ( $M$. brevicaudata- $M$. theresa), with Thylamys macrurus in basal position, forming a trichotomy with $T$. pusillus and the $T$. pallidior-T. venustus group. The last clade is the more speciose one and includes the monophyletic Marmosops (node M; arranged in the same sequence as in the $\mathrm{CO}$ analysis), Lestodelphys, and the large opossums (node $\mathrm{G}$ ). Unexpectedly, the position of the Patagonian Lestodelphys is not close to some group of

jackknife frequencies (cutoff value $=50 \%$ ). Heavy lines denote branches with a decay index of $\geq 4$. Outgroup taxa are indicated with asterisks, and alphabetic labels indicate didelphine clades discussed in the text. 


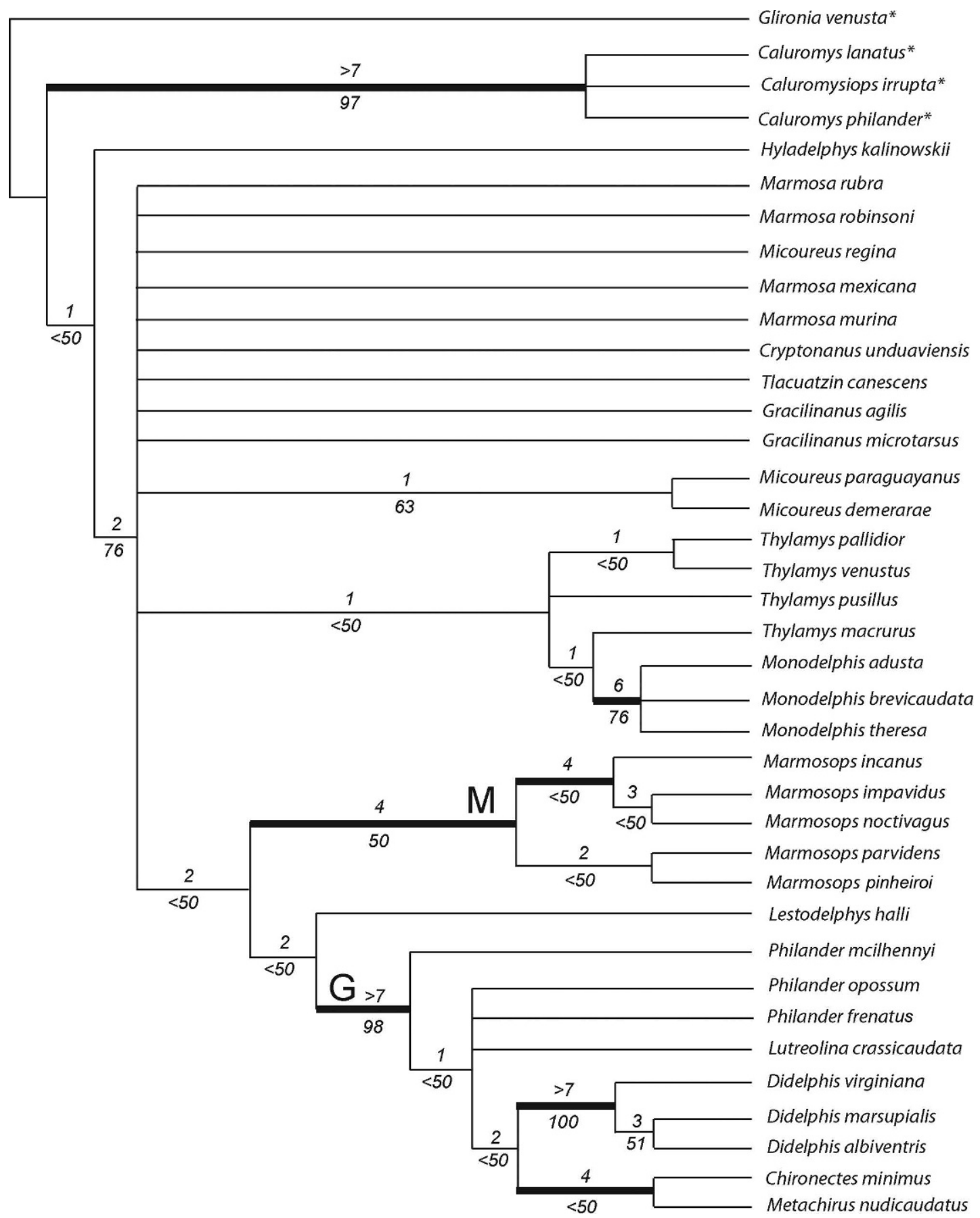

Fig. 34. Strict consensus of seven equally most parsimonious trees resulting from cladistic parsimony analysis of 114 postcranial characters for 38 taxa described in this report, where polymorphic data were treated as transformation series (TS) (see tables 2 and 4 for summary data set characteristics and tree statistics). Numbers above branches refer to absolute Bremer support values $(\geq 1)$. Numbers below branches refer to jackknife frequencies (cutoff value $=50 \%$ ). Heavy lines denote branches with a decay index of $\geq 4$. Outgroup taxa are indicated with asterisks. Alphabetic labels indicate didelphine clades discussed in the text. 
mouse opossums, but as sister of large opossums. In the last group, the topology is almost similar to the one obtained in $\mathrm{CO}$ analysis, but Philander is paraphyletic and Metachirus and Chironectes are sister groups.

\section{Nonmolecular Evidence on Didelphid \\ PHYLOGENY: THE INCLUSION OF Postcranial Characters}

In table 3 are summarized and compared basic statistics for the nonomolecular data set published by Jansa and Voss (2005), the postcranial data presented herein, and the combination of both morphological data sets. Note that both nonmolecular data sets are proportionally similar regarding some basic statistics, that is, as percentages of missing and inapplicable data, parsimony informative characters, and autapomorphies. However, the percentage of ordered multistate characters is higher in the postcranial data set presented here. Combining both nonmolecular data sets analyzed with $\mathrm{CO}$ polymorphic entries, I recovered 120 most parsimonious trees (723 steps, CI $=0.33$, RI $=0.68$ ) whose strict consensus is shown in figure 35 . In this topology, the caluromyines (Caluromys + Caluromysiops) form a monophyletic group, with both species of Caluromys as sister taxa. The intermediate position of Hyladelphys between caluromyines and didelphines appears here again (table 6). Up in the tree, the topology appears as a deep dichotomy, which includes the remaining didelphids arranged basically in two monophyletic clades. In the first group, Tlacuatzin is sister taxon of the group GracilinanusCryptonanus-Chacodelphys-Thylamys-Lestodelphys (node B). In this clade, Gracilinanus and Cryptonanus are respectively recovered as monphyletic groups, and Lestodelphys is nested in the paraphyletic Thylamys (node A) in an unresolved trichotomy with $T$. venustus and $T$. pallidior. $T$. pusillus and $T$. macrurus are successively basal to this group. The second clade is formed by a basal polytomy including Micoureus regina, Marmosa murina, M. lepida, M. mexicana, and M. robinsoni; the group resolved as the sequence Marmosa rubra (Micoureus paraguayanus- $M$. demerarae)); and the group conformed by the monophyletic Marmosops (node M) and the clade formed by the monophyletic Monodelphis and the large opossums as sister groups (node O). The species of Marmosops are split in two sister clades, one formed by (Marmosops incanus (M. impavidus-M. noctivagus)), and another including $M$. parvidensM. pinheiroi. The four species of Monodelphis considered in the morphological analysis are clustered in a well-supported monophyletic clade where $M$. brevicaudata and $M$. emiliae form a pair and $M$. adusta and $M$. theresa complete a trichotomy. The clade formed by the large opossums (node G) includes the monophyletic Didelphis, Philander (sister of the monotypic Lutreolina, node E), and Chironectes and Metachirus successively arranged in increasingly basal positions (nodes $F$ and $G$, respectively). The three species of Didelphis form an unresolved polytomy, and the species of Philander are resolved as ( $P$. mcilhennyi ( $P$. opossum- $P$. frenatus)).

A heuristic search of all morphological data sets with polymorphic entries analyzed as TS resulted in six most parsimonious trees of 926 steps $(\mathrm{CI}=0.29$, $\mathrm{RI}=0.65)$. The strict consensus (fig. 36) results in a barely less resolved topology than the $\mathrm{CO}$ analysis (table 5). Deep in the tree, Hyladelphys is placed in its typical intermediate position, and Marmosa lepida, M. rubra, and $M$. robinsoni are successively arranged basal to the remaining didelphids, which are split in two groups: the clade conformed by the sister species Micoureus paraguayanus-M. demerarae, and a polytomy including the remaining didelphine species. Seven natural groups can be recognized in this unresolved polytomy. Three out of seven monophyletic groups consist of pairs of species: Gracilinanus agilis-G. microtarsus, G. aceramarcae-G. emiliae, and Cryptonanus unduaviensis-C. chacoensis. The four remaining natural groups include the complex Thylamys-Lestodelphys (node A), the monophyletic Marmosops (node $\mathrm{M}$ ) and Monodelphis, and the large opossums (node $\mathrm{G}$ ) in the same topology as in the $\mathrm{CO}$ analysis. However, in the TS analysis Monodelphis adusta and M. theresa are sister species, Philander is recovered as an unresolved trichotomy, and Didelphis is recovered in the arrangenment (Didelphis virginiana (D. albiventris-D. marsupialis). 


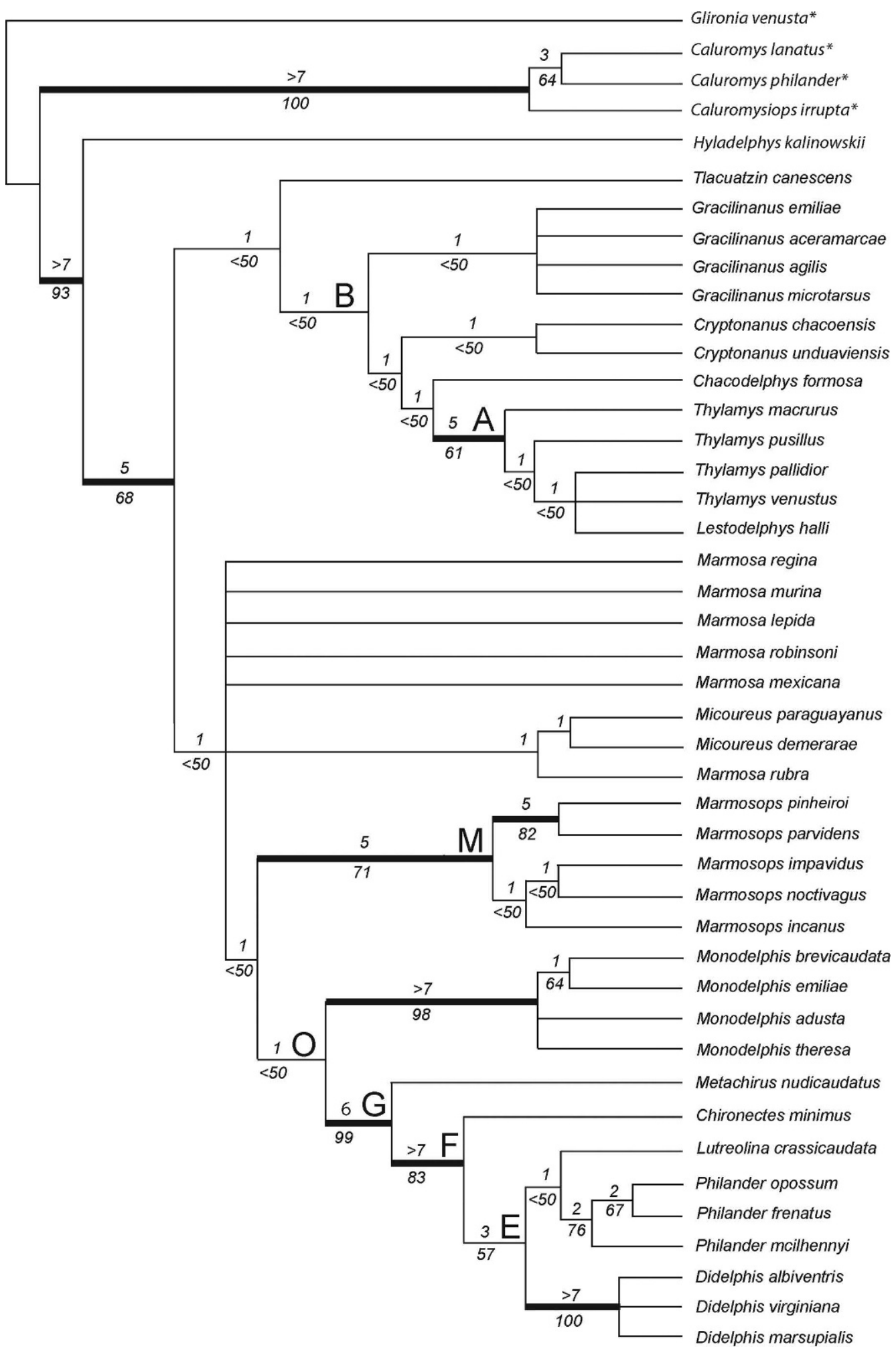




\section{COMBined AnAlysis}

Parsimony analysis combining the new postcranial evidence with the nonmolecular characters previously defined, plus IRBP, DMP-1, and RAG-1 sequences analyzed with $\mathrm{CO}$ polymorphic entries, resulted in two most parsimonious trees (6203 steps, $\mathrm{CI}=0.61$, RI $=0.80)$. The strict consensus topology (fig. 37) resembles, in some positions, the didelphid relationships obtained in the combined evidence by Jansa and Voss (2005: fig. 1D) and Gruber et al. (2007: fig. 2), although the deep branch topology differs remarkably. The relationships within the outgroup are similar to the one obtained with nonmolecular evidence (i.e., Caluromys and Caluromysiops forming a monophyletic group, and both species of Caluromys as sister taxa). Hyladelphys kept its intermediate position between caluromyines and didelphines. From this point of the tree, two traditionally recognized groups in the didelphine subfamily are recovered as monophyletic groups: the $2 \mathrm{n}=22$ large opossums (node F), and the mouse opossums (node L, although including Metachirus). Among the large opossums, the two polytypic living genera (Didelphis and Philander) are recovered as monophyletic and sister groups (node D). Lutreolina and Chironectes are successively arranged in increasingly basal positions (nodes $\mathrm{E}$ and $\mathrm{F}$, respectively). The remaining taxa are split into three diverse clades. The first one contains the monophyletic Monodelphis, resolved in the sequence ( $M$. emiliae (Monodelphis theresa (M. adusta-M. brevicaudata))), and Chacodelphys as sister taxa. More nested in the tree, the complex Micoureus-Marmosa (node I) is resolved on the monophyletic Micoureus in the sequence (Micoureus regina (M. demerarae-M. paraguayanus)), and Marmosa as paraphyletic. Marmosa lepida and M. murina are successively basal to Micour- eus. On the other hand, this group is sister of the group (Marmosa rubra (M. mexicana-M. robinsoni)). The last clade (node $\mathrm{C}$ ) includes the paraphyletic Marmosops, Metachirus, and the complex Thylamys-Lestodelphys (node A), sister of Gracilinanus-Cryptonanus group (node B). Although Marmosops is recovered as monophyletic in other analyses (e.g., nonmolecular and previous combined analyses), the complete evidence presented here recovered the species in two different clades. Three species are resolved in the grouping (Marmosops incanus (M. impavi$d u s-M$. noctivagus), and the sister taxa $M$. parvidens and $M$. pinheiroi are clustered with Metachirus. The species of Thylamys are clustered in a monophyletic group as an unresolved polytomy, although $T$. venustus is placed basal in relation to the remaining species of the genus. As in other results, Lestodelphys is sister of Thylamys. Both species of Cryptonanus (chacoensis and unduaviensis) are recovered as sister taxa, clustered with the monophyletic Gracilinanus (node N), which shows the sequence (Gracilinanus emiliae ( $G$. aceramarcae ( $G$. agilis- $G$. microtarsus))).

A heuristic search of combined data sets with polymorphic entries analyzed as TS resulted in four most parsimonious trees (6464 steps, $\mathrm{CI}=0.59, \mathrm{RI}=0.79)$. The strict consensus (fig. 38) under this parameter is notably less resolved than the topology obtained in $\mathrm{CO}$ analysis (table 5), and some differences can be detected. In this scheme, the position of the $2 n=22$ large opossums (node F), which under $\mathrm{CO}$ treatment is a sister group of the mouse opossums (fig. 37), is clustered with most of the mouse opossums in a polytomy where the nodes $\mathrm{B}$ and $\mathrm{C}$ (observed in $\mathrm{CO}$ analysis) are not recovered (figs. 37, 38). Another minor difference with $\mathrm{CO}$ analysis is the inverted position of Monodelphis theresa and M. emiliae.

Fig. 35. Strict consensus of 120 equally most parsimonious trees resulting from cladistic parsimony analysis of 185 morphological characters for 44 didelphid taxa, where polymorphic data were treated as composite entries (CO) (see tables 2-4 for summary data set characteristics and tree statistics). Numbers above branches refer to absolute Bremer support values $(\geq 1)$. Numbers below branches refer to jackknife frequencies (cutoff value $=50 \%$ ). Heavy lines denote branches with a decay index of $\geq 4$. Outgroup taxa are indicated with asterisks. Alphabetic labels indicate didelphine clades discussed in the text. 


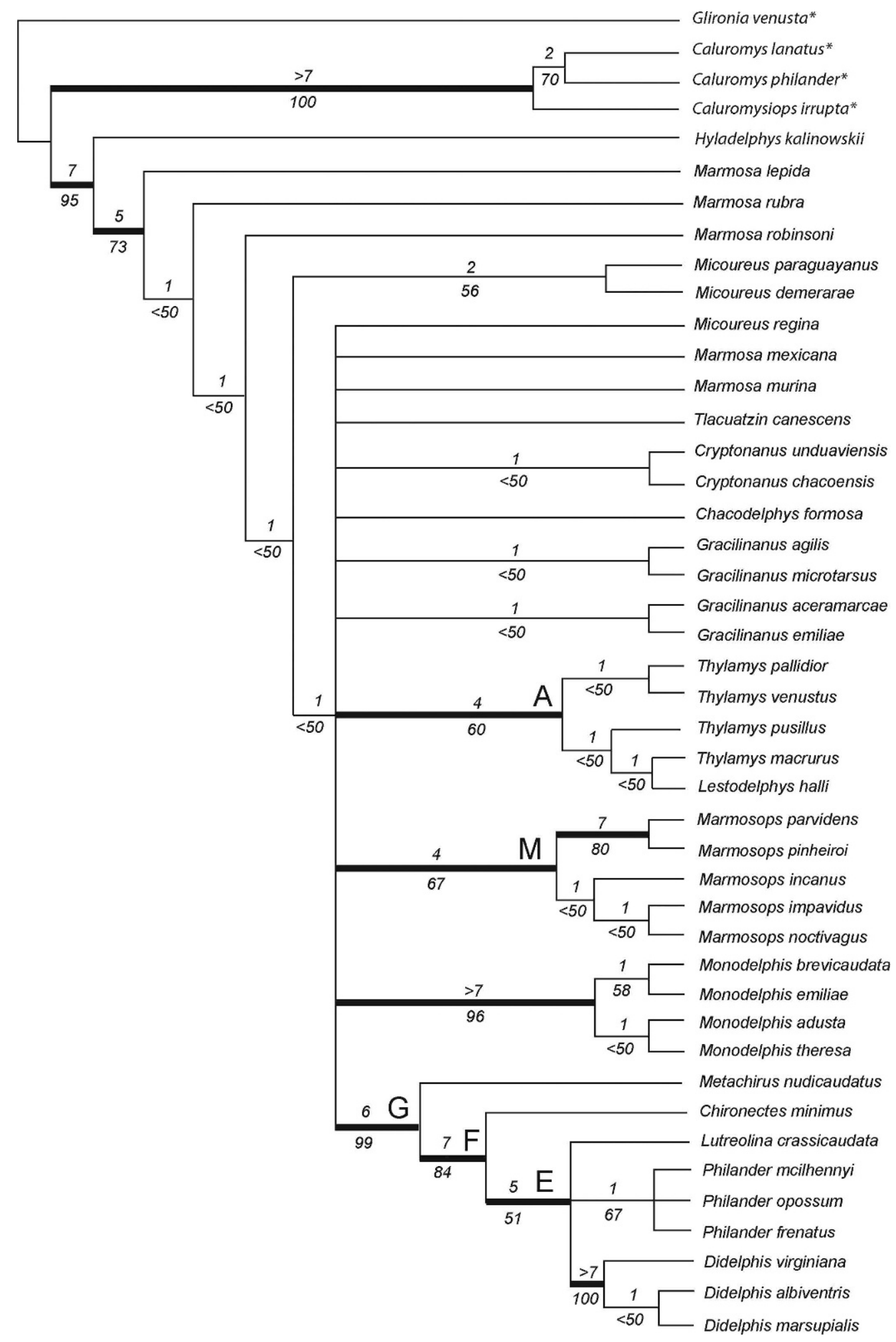




\section{NODAL SUPPORT}

Bremer support values obtained from the postcranial evidence are, in general terms, low. For instance, in the $\mathrm{CO}$ analysis (fig. 33), 21 nodes (68\% of resolved nodes) collapse in trees that are one step longer, 4 additional nodes (13\%) collapse in trees that are two steps longer, whereas 2 more nodes $(6 \%)$ collapse in trees that are three steps longer. Only four nodes (13\% of the total) have a decay index $\geq 4$ (table 5). Although the TS analysis has less resolved ingroup nodes in the consensus tree (fig. 34; table 5), the amount of well-supported nodes is higher (table 5). Five nodes (28\%) collapse in trees that are one step longer, five additional nodes $(28 \%)$ collapse in trees two steps longer, and two nodes $(11 \%)$ collapse in trees three steps longer. Lastly, the remaining six nodes (14\%) have a decay index $\geq 4$ (table 5). Resampling values were also low: in $\mathrm{CO}$ analysis 22 nodes (71\%) have jackknife values below $50 \%$, and 6 nodes (19\%) have jackknife values between $50 \%$ and $85 \%$, while the remaining 3 nodes $(9 \%)$ have jackknife values higher than $85 \%$. In the TS postcranial analysis 11 nodes $(61 \%)$ have jackknife values below 50\%, 4 nodes (22\%) have jackknife values between 50\% and $85 \%$, while only 2 nodes (11\%) have jackknife values higher than $85 \%$.

Nodal support values for morphology of the total tree are slightly higher. In the $\mathrm{CO}$ analysis (fig. 35), 17 nodes (59\%) collapse in trees that are one step longer, 2 additional nodes $(7 \%)$ collapse in trees two steps longer, 1 node $(3 \%)$ collapses in trees that are three steps longer, and 9 nodes (31\%) have a decay index $\geq 4$ (table 5). The consensus tree of the TS analysis (fig. 36) is less resolved than the CO analysis, although in the TS analysis there is one more well-supported node than for the $\mathrm{CO}$ results. Twelve nodes (44\%) collapse in trees that are one step longer, four additional nodes (15\%) collapse in trees two steps longer, and only one node (4\%) collapses in trees three steps longer. The remaining 10 nodes $(37 \%)$ have a decay index $\geq 4$ (table 5). Similarly, resampling values are slightly higher in morphology of the total tree: in the $\mathrm{CO}$ analysis 16 nodes $(55 \%)$ have jackknife values below 50\%, 8 nodes $(27 \%)$ have jackknife values between $50 \%$ and $85 \%$, while the remaining 4 nodes $(14 \%)$ have jackknife values higher than $85 \%$. In the TS morphology/total analysis, 15 nodes (55\%) have jackknife values below 50\%, 8 nodes (29\%) have jackknife values between $50 \%$ and $85 \%$, and the remaining 4 nodes $(15 \%)$ have jackknife values higher than $85 \%$.

Nodal support values for the combined $\mathrm{CO}$ analysis indicate that most of 38 resolved ingroup nodes in the consensus tree (fig. 37) are moderately well supported. Only eight nodes $(21 \%)$ collapse in trees one step longer, one additional node $(3 \%)$ collapses in trees two steps longer, and three more nodes (8\%) collapse in trees three steps longer. The remaining 26 nodes (57\%) have a decay index $\geq 4$ (table 5). The consensus tree of the TS analysis (fig. 38) shows less resolved ingroup nodes than for the $\mathrm{CO}$ analysis. Only three nodes $(10 \%)$ collapse in trees one step longer, two additional nodes (6\%) collapse in trees two steps longer, and six more nodes (19\%) collapse in trees three steps longer. The remaining 20 nodes (64\%) have a decay index $\geq 4$ (Table 5). Similarly, resampling values were relatively high both in the $\mathrm{CO}$ and TS combined analyses. In the $\mathrm{CO}$ analysis 10 nodes (26\%) have jackknife values below $50 \%, 6$ nodes $(16 \%)$ have jackknife values between $50 \%$ and $85 \%$, and the remaining 22 nodes $(58 \%)$ have jackknife values higher than $85 \%$. On the other hand, in the TS combined analysis 16 nodes (52\%) have jackknife values below 50\%, 8 nodes $(26 \%)$ have jackknife values between $50 \%$ and $85 \%$,

Fig. 36. Strict consensus of six equally most parsimonious trees resulting from cladistic parsimony analysis of 185 morphological characters for 44 didelphid taxa, where polymorphic data were treated as transformation series (TS) (see tables 2 and 4 for summary data set characteristics and tree statistics). Numbers above branches refer to absolute Bremer support values $(\geq 1)$. Numbers below branches refer to jackknife frequencies (cutoff value $=50 \%$ ). Heavy lines denote branches with a decay index of $\geq 4$. Outgroup taxa are indicated with asterisks. Alphabetic labels indicate didelphine clades discussed in the text. 


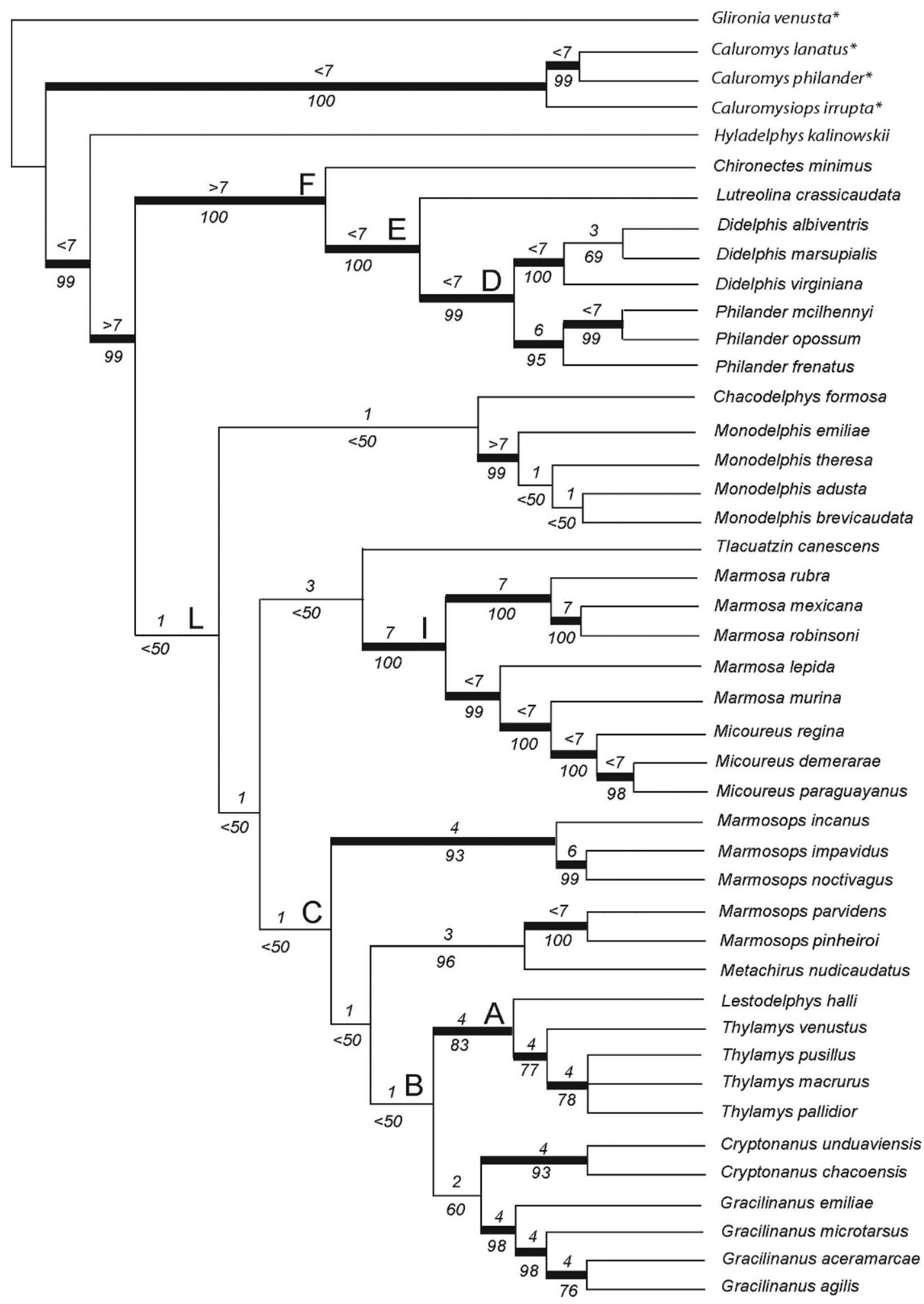


and the remaining 7 nodes $(23 \%)$ have jackknife values higher than $85 \%$.

\section{DISCUSSION}

\section{EFFECTS OF DifFERENT CODINGS OF POLYMORPHIC DATA}

The kind of treatment of polymorphic characters may have a significant impact on phylogenetic analyses. Different methods for dealing with polymorphism may lead to very different estimations of phylogeny, even when relationships are strongly supported by one or more methods (Wiens, 1999). The abundance and impact of polymorphic characters are especially clear for closely related species, but the application of different criteria for analyzing polymorphic data may affect higher level relationships as well. In this sense, although different codings of polymorphic postcranial characters in didelphids produced topologies in general not contradictory, some discrepancies were evident. The principal difference was the loss of resolution of the TS analysis compared to the $\mathrm{CO}$ analysis, with the consistency index and retention index being slightly higher in the $\mathrm{CO}$ analysis (table 5). In the postcranial results, the nodes weakly supported in the $\mathrm{CO}$ analysis collapsed in the TS topology (figs. 33, 34). However, more resolution is expected for the additional phylogenetic information with TS coding (Mabee and Humpries, 1993). Different codings caused little impact on the nodal support, since in both topologies the number of nodes with high decay index $(\geq 4)$ was similar in all analyses, except in the combined analysis considering RAG-1 (table 5), where the CO coding had more well-supported nodes. An inverted bias on nodal support and resolution was observed in a combined analysis in Oryzomyini rodents performed by Weksler
(2006). In the case of didelphids, despite the differences in resolution in the combined total evidence, most relationships obtained in the TS analysis are not contradicted by the $\mathrm{CO}$ analysis, except for two punctual cases: the inverted position of Monodelphis emiliae and $M$. adusta, and the basal position of the Marmosa-Micoureus group (node I) in TS analysis (being instead sister of node $\mathrm{C}$ in the $\mathrm{CO}$ analysis, figs. 37,38 ).

In the total morphology analysis, the effects of different codings on polymorphic data were similar with respect to the postcranial-only data set: the topology obtained with the CO coding analysis is notably better resolved than the TS coding analysis (figs. 35, 36), although the values of Bremer support are in general rather similar (table 5). Contrary to the congruence observed in the topology obtained by both kinds of coding in the potscranial-only analysis, the low resolved topology observed with the TS coding in the total morphology analysis differs considerably in some positions with regard to the $\mathrm{CO}$ coding analysis. In the TS coding total morphology analysis, the change of position of Marmosa lepida and M. rubra is unexpected for different reasons. In the first case, all postcranial characters are missing, and the remaining morphological characters do not show polymorphic entries (see Voss and Jansa, 2003: appendix 5). In Marmosa rubra, although $95 \%$ of the postcranial characters were scored (table 4), there were no polymorphisms since the sample consisted of only one specimen (see appendix 1 ), and the remaining morphological characters do not show any polymorphic entries (see Voss and Jansa, 2003: appendix 5). Lastly, the change observed in Marmosa robinsoni is perhaps a consequence of the high polymorphism present in postcranial morphology (table 2; appendix 2). Other

Fig. 37. Strict consensus of two equally most parsimonious trees resulting from cladistic parsimony analysis of combined morphological and genetic data (i.e., all morphology, IRBP, DMP-1, and RAG-1 sequences) for 44 didelphid taxa, where polymorphic data were treated as composite entries (CO) (see tables 3 and 4 for summary data set characteristics and tree statistics). Numbers above branches refer to absolute Bremer support values $(\geq 1)$. Numbers below branches refer to jackknife frequencies (cutoff value $=50 \%$ ). Heavy lines denote branches with a decay index of $\geq 4$. Outgroup taxa are indicated with asterisks. Alphabetic labels indicate didelphine clades discussed in the text. 


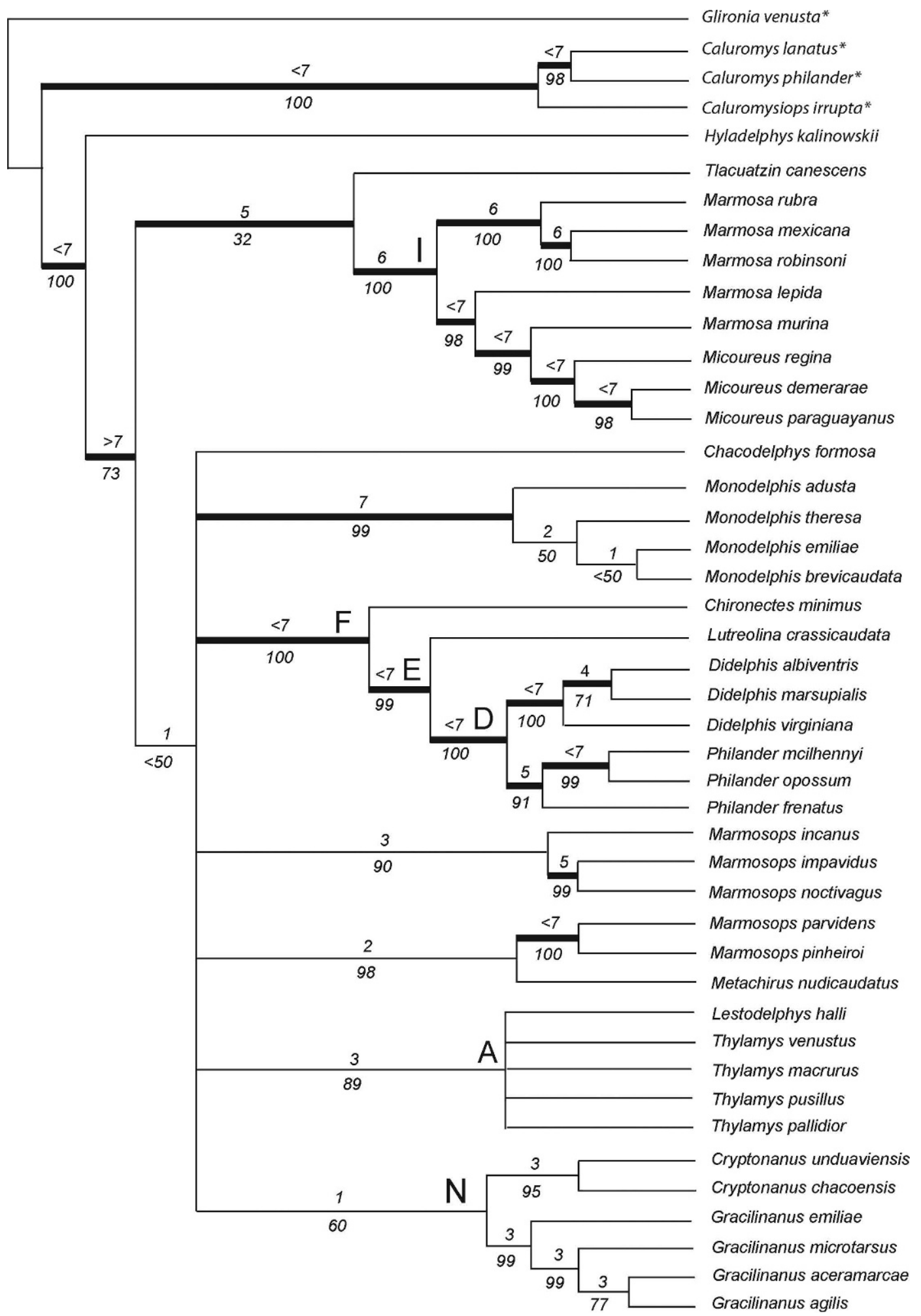


differences can be noted between both kinds of coding, such as the monophyletic condition of Gracilinanus in $\mathrm{CO}$ coding total morphology analysis, the changing position of Thylamys macrurus and T. pusillus, and the relationship among the species of the monophyletic Didelphis and Philander. Despite the differences in deep branch topology, the wellsupported clades in $\mathrm{CO}$ coding total morphology analysis were all recovered in TS coding morphology-total analysis as well (i.e., the monophyly of Monodelphis, Philander, Marmosops, and Didelphis, the relationship of large opossums [nodes E, F, and G], and the relationship of Thylamys-Lestodelphys [node A]). Similarly to postcranial-only analysis, in the case of total morphology, the TS coding analysis seems not to contribute to the retention of more phylogenetic information.

As described in the results, the topologies obtained including the genetic evidence (i.e., combined analysis) are in general highly resolved and better supported than the morphology-only analyses. Despite the fact that the topologies from both kind of codings of polymorphic data are significantly congruent in some aspects, the clustering of the mouse opossums and the better resolution applying CO coding analysis (fig. 37) are interesting. The mouse opossums are the most speciose group in the didelphid living radiation, and the genera currently recognized (sensu Gardner, 2005) are not always recovered as natural groups in the cladistic context. Contrasting with the large opossums, which were considered as a monophyletic group based in a diverse array of previous evidence, the mouse opossums were partially supported only in a morphological framework (e.g. Creighton, 1984; Reig. et al., 1987; Goin, 1995; Flores, 2003). Here, the addition of postcranial evidence to the previous nonmolecular and genetic evidence causes the moderately supported clustering of the mouse opossums in a group just in $\mathrm{CO}$ coding analysis (although Metachirus is nested in the group when RAG-1 is included; fig. 37; appendix 3). However, the group is paraphyletic in all previous nonmolecular and molecular (IRBP, DMP-1, RAG-1) analyses (Kirsch and Palma, 1995; Patton et al., 1996; Jansa and Voss, 2000, 2005; Voss and Jansa, 2003; Voss et al., 2005; Jansa et al., 2006; Gruber et al., 2007), and even in all remaining analyses of this report. In this sense, applying different criteria for the treatment of morphological polymorphic data, the relationships and monophyly of the mouse opossums are strongly affected.

\section{EFFECT OF THE INCLUSION OF POSTCRANIAL Characters in Previous Nonmolecular AND COMBINED Hypotheses}

Nonmolecular Hypotheses: Comparing the topology of nonmolecular evidence illustrated by Jansa and Voss (2005: fig. 1C), the inclusion of the postcranial data set causes considerable changes and better resolution in topology and support values (figs. 35, 36; tables 5-7). As mentioned above, the intermediate position of Hyladelphys is consistent in postcranial-only and combined analyses (table 6). The trichotomy conformed by the monophyletic Didelphis and Philander, as well as the monotypic Lutreolina (node E), is kept both in CO and TS analyses of total morphology evidence, as well as the monophyly of Monodelphis and its sister relationship with large opossums (node $\mathrm{O}$ in the $\mathrm{CO}$ analysis, fig. 35), the relationship of Lestodelphys-Thylamys (node A), and the monophyly of Marmosops (node M), Gracilinanus, and Cryptonanus (although the TS coding analysis produces the rupture of

Fig. 38. Strict consensus of four equally most parsimonious trees resulting from cladistic parsimony analysis of morphological and genetic data (i.e., all morphology, IRBP, DMP-1, and RAG-1 sequences) for 44 didelphid taxa, where polymorphic data were treated as transformation series (TS) (see tables 3 and 4 for summary data set characteristics and tree statistics). Numbers above branches refer to absolute Bremer support values $(\geq 1)$. Numbers below branches refer to jackknife frequencies (cutoff value $=50 \%$ ). Heavy lines denote branches with a decay index of $\geq 4$. Outgroup taxa are indicated with asterisks. Alphabetic labels indicate didelphine clades discussed in the text. 
TABLE 7

Nodes Recovered under Different Analyses and Values of Absolute Bremer Support and Jackknife Frequencies ${ }^{\text {a }}$

\begin{tabular}{|c|c|c|c|c|c|c|c|c|}
\hline Node & 1 & 2 & 3 & 4 & 5 & 6 & 7 & 8 \\
\hline A & & & $5 / 61$ & $4 / 60$ & $4 / 83$ & $3 / 89$ & $4 / 83$ & $5 / 86$ \\
\hline B & & & $1 /<50$ & & $1 /<50$ & & $1 /<50$ & $2 /<50$ \\
\hline $\mathrm{C}$ & & & & & $1 /<50^{\mathrm{b}}$ & & $1 /<50$ & $2 /<50$ \\
\hline $\mathrm{D}$ & & & & & $>7 / 99$ & $>7 / 100$ & $4 / 73$ & $4 / 78$ \\
\hline $\mathrm{E}$ & $1 /<50$ & & $3 /<50$ & $6 /<50$ & $>7 / 100$ & $>7 / 99$ & $>7 / 97$ & $>7 / 99$ \\
\hline $\mathrm{F}$ & & & $>7 / 66$ & $6 / 84$ & $>7 / 100$ & $>7 / 100$ & $>7 / 100$ & $>7 / 100$ \\
\hline G & $>7 / 98$ & $>7 / 98$ & $6 / 83$ & $7 / 99$ & & & $5 / 99$ & 7/99 \\
\hline $\mathrm{H}$ & & & & & & & & $2 /<50$ \\
\hline I & & & & & $7 / 100$ & $6 / 100$ & 7/99 & $>7 / 94$ \\
\hline $\mathbf{J}$ & & & & & & & $1 /<50$ & $2 /<50$ \\
\hline $\mathrm{L}$ & & & & & $1 /<50^{\mathrm{b}}$ & & $4 / 75^{\mathrm{c}}$ & \\
\hline M & $4 / 50$ & $4 / 50$ & $5 / 71$ & $5 / 67$ & & & $5 / 97$ & $6 / 98$ \\
\hline $\mathrm{N}$ & & & & & $2 / 60$ & $1 / 50$ & $1 /<50$ & $2 /<50$ \\
\hline $\mathrm{O}$ & $1 /<50$ & & $1 /<50$ & & & & & \\
\hline
\end{tabular}

${ }^{\text {a }}$ Letters indicate the nodes labeled as in figures 33-40. Each column corresponds to different analyses described and compared in the text. 1, Postcranial data, CO coding for polymorphic entries; 2, postcranial data, TS coding; 3, all nonmolecular data set (including postcranium), CO coding; 4, all nonmolecular dataset (including postcranium), TS coding; 5, combined data (including postcranium), CO coding; 6, combined data (including postcranium), TS coding; 7, combined data (including postcranium), RAG-1 eliminated, $\mathrm{CO}$ coding; 8, combined data (including postcranium), RAG-1 eliminated, TS coding.

bIncluding Metachirus.

${ }^{\mathrm{c}}$ Excluding Metachirus.

the monophyly of Gracilinanus, fig. 36). However, some alterations in the topology can be detected by including the postcranial characters. In the $\mathrm{CO}$ coding analysis (fig. 35), both species of Cryptonanus (chacoensis and unduaviensis) appear as a monophyletic group sister to the clade Chacodelphys-node A (Thylamys-Lestodelphys), and the species of Gracilinanus are also clustered as monophyletic basal in node $\mathrm{B}$. The monophyly of node $\mathrm{B}$ in the $\mathrm{CO}$ coding analysis (fig. 35) is recovered by including the postcranial evidence. In this sense, consideration of the postcranial characters on the morphological evidence previously defined (Jansa and Voss, 2005) is consistent with the genetic evidence, since clustering of the monophyletic genera Gracilinanus and Cryptonanus with node A was obtained using only genetic and combined evidence (Jansa and Voss, 2005; Gruber et al., 2007). The position of Metachirus is also altered when the postcranial evidence is included, since this monotypic taxon is basal to the large $2 \mathrm{n}=22$ opossums in the total morphology analysis (node $\mathrm{G}$ in figs. 35, 36), whereas it is located as the sister taxon of the group consisting of
$2 \mathrm{n}=22$ opossums-Monodelphis in the morphological analysis excluding the postcranial characters (see Jansa and Voss, 2005: fig. 1C; table 7). Although Metachirus shows a particular mode of locomotion and some postcranial autapomorphies (see the character descriptions and appendix 2), its close relationship with the $2 \mathrm{n}=22$ large opossums (node $\mathrm{G}$ in figs. 35,36 ) is well supported by postcranial morphology (table 7). Similarly, the inclusion of postcranial evidence notably affects the position of the recently redescribed Tlacuatzin canescens. This taxon appears in a basal polytomy together with some species of Marmosa and Micoureus in the morphological evidence of Jansa and Voss (2005: fig. 1C). A similar position is obtained when the TS coding criterion is applied for polymorphic characters in the total morphology analysis (fig. 36), but when the postcranial data set is included in the $\mathrm{CO}$ coding analysis, this taxon is placed as sister to node $\mathrm{B}$, although with low support (fig. 35).

The relationship Thylamys-Lestodelphys (node A) and the paraphyly of Thylamys are also kept when postcranial evidence is considered (table 7), but the position of this 
clade in the total morphology consensus tree is remarkably different. In the topology obtained by Jansa and Voss (2005: fig. 1C) this clade is located as sister of the Monodelphis-large opossums group (node $\mathrm{O}$ ), whereas when including the postcranial evidence this group appears as forming part of node $\mathrm{B}$ in the $\mathrm{CO}$ coding analysis (fig. 35). The monophyly of Marmosops (node M) is also kept, but its position is different when the postcranial characters are considered. When omitting postcranial evidence, its position is basal in the clade that includes the large opossums, Monodelphis (node O), and the complex Thylamys-LestodelphysChacodelphys (Jansa and Voss, 2005: fig. 1C). Nonetheless, in the $\mathrm{CO}$ coding total morphology analysis this monophyletic genus appears as sister to node $\mathrm{O}$ (node $\mathrm{G}$ and Monodelphis, fig. 35).

Although the parsimony analysis considering only the postcranial data set resulted in a well-resolved strict consensus but with lower consistency and retention indices compared to the total morphology analysis (i.e., the postcranial characters defined here and the 71 nonmolecular characters defined by Voss and Jansa, 2003; see tables 5, 7; figs. 33, 35), several relationships (which are recovered in both analyses separately) are kept with the inclusion of postcranial evidence (tables 6, 7): the intermediate position of Hyladelphys between caluromyines and didelphines is recovered in all morphological analyses, as is the monophyly of Monodelphis, Marmosops (node M), and the large opossums (Didelphis, Philander, Lutreolina, Chironectes, and $\mathrm{Me}$ tachirus [node G]). Several nodes recovered by including genetic evidence (Jansa and Voss, 2005; Jansa et al., 2006; Gruber et al., 2007) were not found based on morphological evidence omitting postcranial characters. As mentioned above, the inclusion of postcranial evidence also produces better resolution. Nodes B and G (fig. 35) specifically are also recognized in a more inclusive morphological data set, with the postcranial characters concatenated to the previously defined nonmolecular evidence (table 7).

Hypotheses BASEd on Combined EviDENCE: The inclusion of postcranial characters in the combined data set performed by Jansa and Voss (2005), Jansa et al. (2006), and Gruber et al. (2007) causes some interesting alterations in the resulting hypotheses (table 7). The general topology (figs. 37, 38) shows several congruences with diverse aspects of molecular (IRBP, DMP-1) and nonmolecular evidence (table 8). The recent inclusion of RAG-1 sequences (Gruber et al., 2007: figs. 1,2) resulted in the wellsupported clustering of distantly related clades based on profuse evidence: clade B (Thylamys-Cryptonanus-Gracilinanus) as sister of clade I (Marmosa-Micoureus complex). However, the addition of postcranial evidence to the supermatrix analyzed by Gruber et al. (2007) did not recover this apparently spurious clade (figs. 37, 38). The homoplasy caused by the convergence in CG content on the third position in RAG-1 sequences (Gruber et al., 2007) is hidden by the effect of the phylogenetic information coming from the postcranial evidence, independent of the treatment applied to polymorphic characters.

Another example of the influence of postcranial data on previously combined data sets is the position of Hyladelphys (table 6). As mentioned before, this monotypic and recently recognized genus was located in a basal position, intermediate to didelphines and caluromyines, based on profuse molecular and morphological support (Jansa and Voss, 2005). The recent inclusion of RAG-1 sequences alters the typical phylogenetic position of this taxon, being sister to the already mentioned and apparently spurious clade (B+ I in Gruber et al., 2007: fig. 2), although with low support values. The inclusion of postcranial characters in the combined data set (even including the RAG-1 sequences and its third positions) replaces the typical intermediate position of Hyladephys (figs. 37, 38) between caluromyines and didelphines.

Although the combined evidence incorporating the postcranial characters shows some relationships congruent with earlier evidence (tables 7, 8), the position of the cursosaltatorial Metachirus and the recently described Tlacuatzin are highly affected, as was also demonstrated in total morphology analyses. In previous molecular and combined analyses (table 8), Metachirus was within the clade conformed by the large opossums (Didelphis, Philander, Lutreolina, and Chironectes; node $\mathrm{G})$, usually in a basal position (see Gruber et 
TABLE 8

Nodes Recovered in Previous Molecular and Combined Evidence

(All combined analyses excluding postcranial evidence. Letters indicate the nodes labeled as in figures 33-40.)

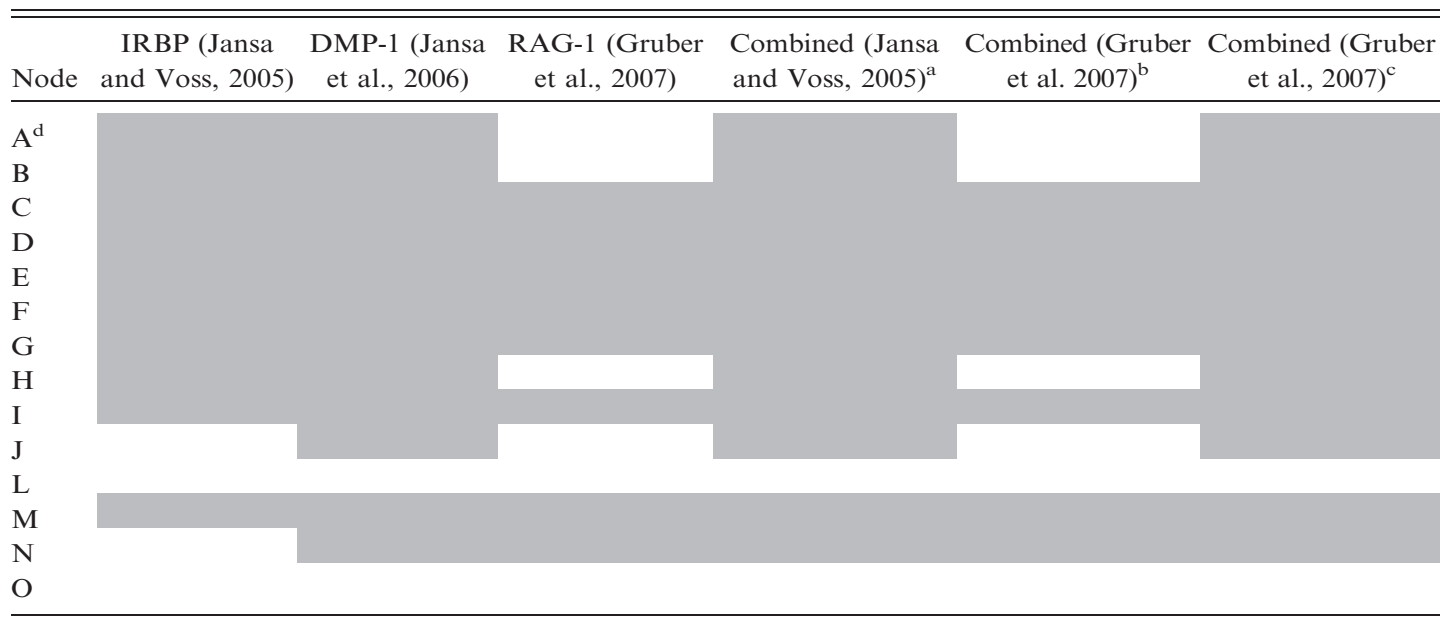

${ }^{\mathrm{a}} \mathrm{RAG}-1$ not included

${ }^{\mathrm{b}} \mathrm{RAG}-1$ included.

${ }^{\mathrm{c}}$ Third position of RAG-1 eliminated.

${ }^{\mathrm{d}}$ Node A (Lestodelphys-Thylamys) is not documented in Gruber et al. (2007) because the RAG-1 sequence data for Lestodelphys is unavailable.

al., 2007; Jansa and Voss, 2005; Jansa et al., 2006). Nonetheless, the addition of postcranial characters to the combined evidence relates Metachirus with Marmosops parvidens- $M$. pinheiroi, nested in the speciose clade labeled $\mathrm{C}$ in figures 37 and 38 (table 7). The inclusion of Metachirus in that group is unlikely in view of the huge amount of phylogenetic information (even postcranial) relating this species to other large opossums. The inclusion of postcranial characters in previously combined evidence (considering also RAG-1 sequences, see appendix 3) apparently affects the presumably true phylogenetic position of Metachirus.

Similarly, consideration of postcranial morphology in combined analyses notably alters the position of Tlacuatzin canescens, which is sister of Monodelphis in all recent combined evidence (see Jansa and Voss, 2005: fig. 1D; Jansa et al., 2006: fig. 5; Gruber et al., 2007: fig. 2). By adding the new data set, Tlacuatzin became sister to the Marmosa-Micoureus complex (node I), a relationship not found in previous analyses. This topology is highly interesting because $T$. canescens was traditionally included in the nonmonophyletic genus Marmosa. However, even when the decay index of this clade is high in the TS and $\mathrm{CO}$ coding combined analyses, the jackknife values in both analyses are notably lower (figs. 37, 38).

As described in the results, the topology obtained by including genetic evidence in the $\mathrm{CO}$ coding analysis is highly resolved and better supported than the morphology-only analyses (tables 7, 8). However, even if the topologies from both kinds of coding of polymorphic characters are considerably congruent in some aspects (figs. 37, 38; table 7), the clustering of the mouse opossums recovered with the $\mathrm{CO}$ coding analysis is remarkable (node $\mathrm{L}$; table 7; although Metachirus is nested in this clade). Here, the addition of postcranial evidence to previous nonmolecular and genetic evidence causes the clustering of the mouse opossums only in the $\mathrm{CO}$ coding analysis, although with most of the support values being low (fig. 37; table 7), which were also paraphyletic in all previous nonmolecular and molecular (table 8) analyses (e.g. Voss and Jansa, 2003; Jansa and Voss, 2000, 2005; Voss et al., 2005; Jansa et al., 2006; Kirsch and Palma, 1995; Patton et al., 1996), and even in all remaining analyses in this report (table 7). 
As discussed above, the addition of postcranial characters recovered some relationships already supported by previous combined analyses (tables 7,8 ), but the resulting topologies are contradictory in some positions. Despite the mentioned incongruences in the positions of Metachirus and Tlacuatzin, the node labeled $\mathrm{O}$, which includes Monodelphis as sister to the large $2 \mathrm{n}=22$ opossums, is recovered when considering the postcranial evidence, but it has not been obseved before in any previous molecular or combined analyses (table 8), except in two analyses in this report (table 7), and in the partial morphological evidence from Jansa and Voss (2005: fig 1C). However, this node is recovered with low support (fig. 38; table 7). Another clear difference concerns the already recognized nodes $\mathbf{J}$ and $\mathrm{H}$ (Voss and Jansa, 2005: fig. 1D; Gruber et al., 2007: fig. 6A-C; Jansa et al., 2006: fig. 4B; table 8), which are well-supported sister groups in previous combined evidence, although this relationship is broken by inclusion of the RAG-1 sequence (figs. 37, 38; see Gruber et al., 2007: fig. 2).

In view of the high convergence of $\mathrm{CG}$ content on the third positions, a more congruent topology was obtained when the third positions of RAG-1 were experimentally eliminated (table 8: Gruber et al., 2007). When omitting the RAG-1 sequence from the combined data set presented here, the relationships obtained are highly congruent with previous evidence recently published, recovering almost all nodes already recognized, even in the TS coding analysis (figs. 39, 40; tables 7, 8). Independent of deep branch differences of the partitioned evidence (depending on the $\mathrm{CO}$ or TS treatment of polymorphic characters), Metachirus recovers its traditional position as sister of the remaining $2 n=22$ large opossums (node $G$ ), and Tlacuatzin is relocated as sister to Monodelphis. The topology obtained with the TS partitioned analysis (fig. 40) is basically similar to the one obtained in the combined analysis by Jansa and Voss (2005: fig. 1D) and some combined topologies obtained by Gruber et al. (2007: fig. 6A-C) and Jansa et al. (2006: fig. 5). Most nodes already documented are recovered: nodes $\mathrm{H}$, $\mathrm{J}, \mathrm{C}, \mathrm{G}, \mathrm{B}$, and I (table 7). However, as in the complete combined data set, the application of different criteria for treatment of polymorphic data in the partitioned analysis strongly affects the relationships and phyletic condition of the mouse opossums, since in the $\mathrm{CO}$ coding analysis node $\mathrm{H}$ is not recovered, and the mouse opossums (node L) are monophyletic although moderately supported (fig. 39; table 7). In this sense, the inclusion of postcranial evidence concatenated to the previous combined data set has notable influence on deep branch topology, depending of the mode of coding polymorphic characters. The partitioned TS combined analysis (excluding RAG-1; fig. 40) recovers all of the topologies already observed (table 7), which indicates that the inclusion of postcranial characters does not contradict the relationships obtained with profuse previous combined evidence (table 8).

The Postcranial Anatomy as Evidence of DidElPHID RELATIONSHIPS AND POSTCRANIAL SyNAPOMORPHIES IN DidelPHinAE

The study of diversity of the cranioskeletal system is one of the most critical areas of research for the understanding of various aspects of behavior and ecological morphology, particularly locomotion and feeding habits in marsupials (Szalay, 1994). Even if the most common conception points toward the close evolutionary relationship between craniodental anatomy and feeding demands, the movements linked to locomotion, posture, and other behavioral patterns are particularly dependent on the musculoskeletal system. Moreover, the skeletal morphology of the most abundantly represented Neogene forms, or extant marsupials, has not been adequately studied from the perspective of evolutionary morphology (Szalay, 1994). Because the skeletal structure is highly correlated with posture, habits, and locomotion, several patterns (both in the axial and appendicular skeleton) have been associated with an apparent functionality. Several recent papers (e.g. Argot 2001, 2002, 2003a, 2003b, 2004a, 2004b; Szalay, 1994; Sears, 2004; Szalay and Sargis, 2001; Weisbecker and Sánchez-Villagra, 2006) have revised the postcranial morphological patterns in mar- 


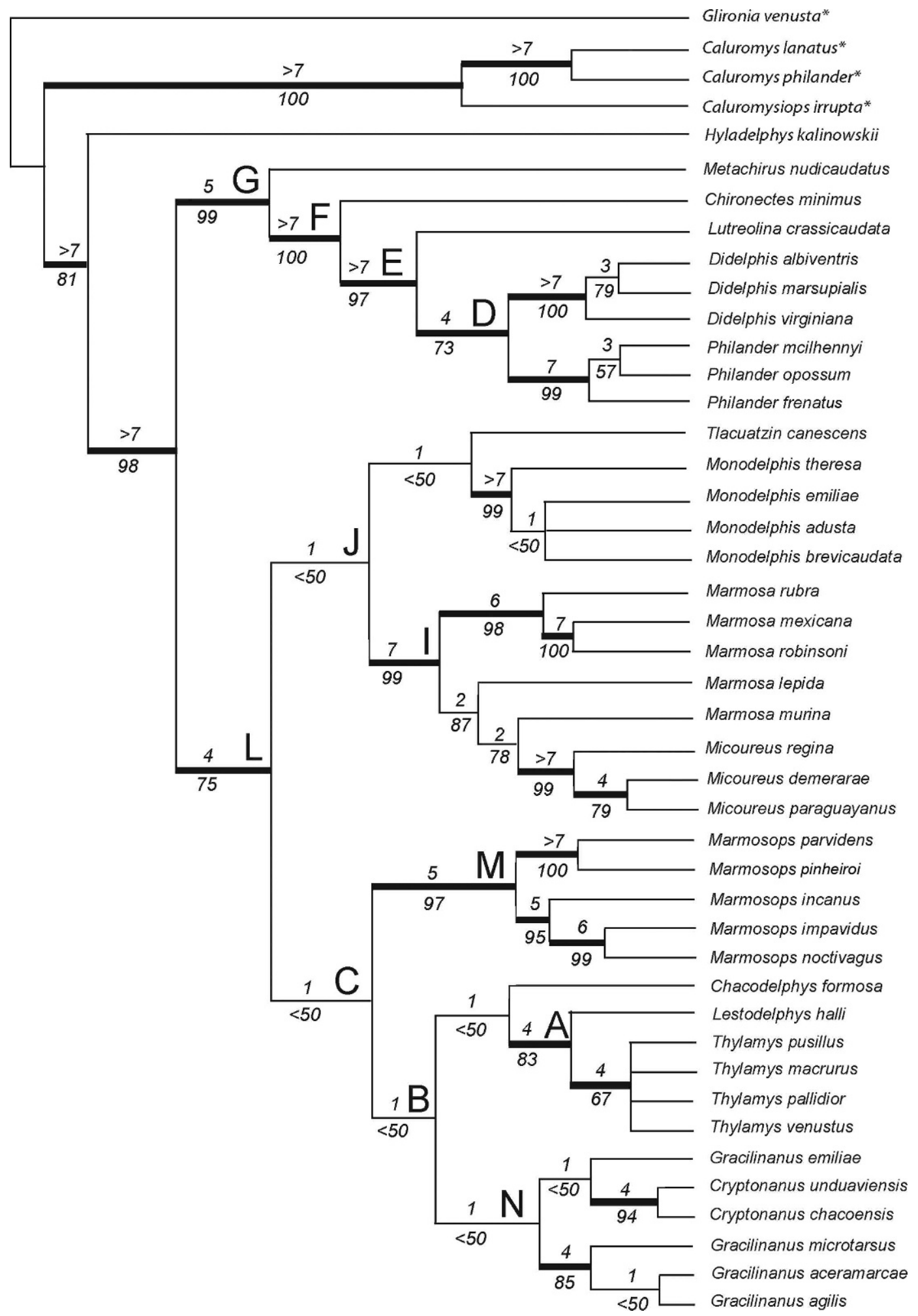


supials and their associated forms-functions on the metatherian postcranium.

The didelphid relationships have been examined with different kinds of data (molecular and morphological), but the application of postcranial characters has never been considered as evidence of didelphid phylogeny in a cladistic frame on a denser taxon sampling. Several postcranial characters defined by Horovitz and Sánchez-Villagra (2003) are highly variable within the didelphid taxa included in this report, although the postcranial unambiguous synapomorphies proposed for Didelphidae in the cited work are also evidenced in all didelphid taxonomic samples considered herein.

Some postcranial topologies recovered in this study (figs. 33, 34) are clearly congruent and noncontroversial with clades already recognized based on other evidence (morphological and molecular; tables 6-8). The postcranial morphology has showed inherent phylogenetic information in recovering some traditionally recognized relationships and monophyletic groups (tables 7, 8; appendix 3). However, the postcranial evidence in didelphids also produces some unusual relationships, a product of the convergence of characters strongly associated with formfunction patterns. In other words, several postcranial characters exhibited the same condition in taxa with analogous locomotion and/or posture patterns, which are clearly linked to specific form-function. For instance, the particular vertical orientation of the astragalonavicular facet of the astragalus (ch. 112[1]) and the depth of the distal calcaneocuboid facet of the calcaneus showed by Metachirus and Monodelphis suggest an increased functional importance of flexionextension of the hindfoot in both terrestrials but not closely related taxa (Szalay, 1994). This is a hint that some atypical relationships obtained are possibly caused by morpholog- ical constraints of form-function in some structures, which can hide the true relationships obtained from other kinds of evidence when the postcranial data set is analyzed separately. This homoplasy is presumably the cause of the low values of consistency index and the poor resolution of the strict consensus (mainly in the TS coding postcranial-only topology, fig. 34) compared to the more resolved trees obtained from the total morphology (fig. 35) and combined evidence (figs. 37-40; table 5).

In the topologies based on the postcranialonly data set, the monophyly of several traditionally recognized polytypic genera (sensu Gardner, 2005), such as Gracilinanus, Marmosa, and Cryptonanus, are not recovered, and some relationships had not been recovered in previous analyses (figs. 33, 34). For instance, the monophyly between the partially terrestrial Thylamys and the highly terrestrial Monodelphis is recovered in the strict consensus of the TS coding postcranial analysis (fig. 34), which is supported by an array of characters clearly related to specific capacities of movements: position of the vertebra where the accessory process is differentiated from the transverse process on T7 (implying an anterior point restricting lateral flexibility; ch. 30[1]), or absence of a longitudinal groove on the lateral surface of the ulna for insertion of $\mathrm{Mm}$. abductor pollicis longus and anconeus (muscles well developed in arboreal forms [Argot, 2001]; ch. 77[0]). In all other topologies obtained here (i.e., total morphology and combined, figs. 35-40), as well as in previous hypotheses (Jansa and Voss, 2000, 2005; Gruber et al., 2007; Voss and Jansa, 2003; Jansa et al., 2006; Reig et al., 1987; Kirsch and Palma, 1995), Monodelphis and Thylamys are clustered in clearly distant clades. Only in the pioneer nonmolecular analysis of Creighton (1984) are both taxa clustered in a mono-

Fig. 39. Strict consensus of six equally most parsimonious trees resulting from cladistic parsimony analysis of morphological and genetic data combined, excluding RAG-1 sequences, for 44 didelphid taxa where polymorphic data were treated as composite entries (CO). Numbers above branches refer to absolute Bremer support values $(\geq 1)$. Numbers below branches refer to jackknife frequencies (cutoff value $=50 \%$ ). Heavy lines denote branches with a decay index of $\geq 4$. Outgroup taxa are indicated with asterisks. Alphabetic labels indicate didelphine clades discussed in the text. 


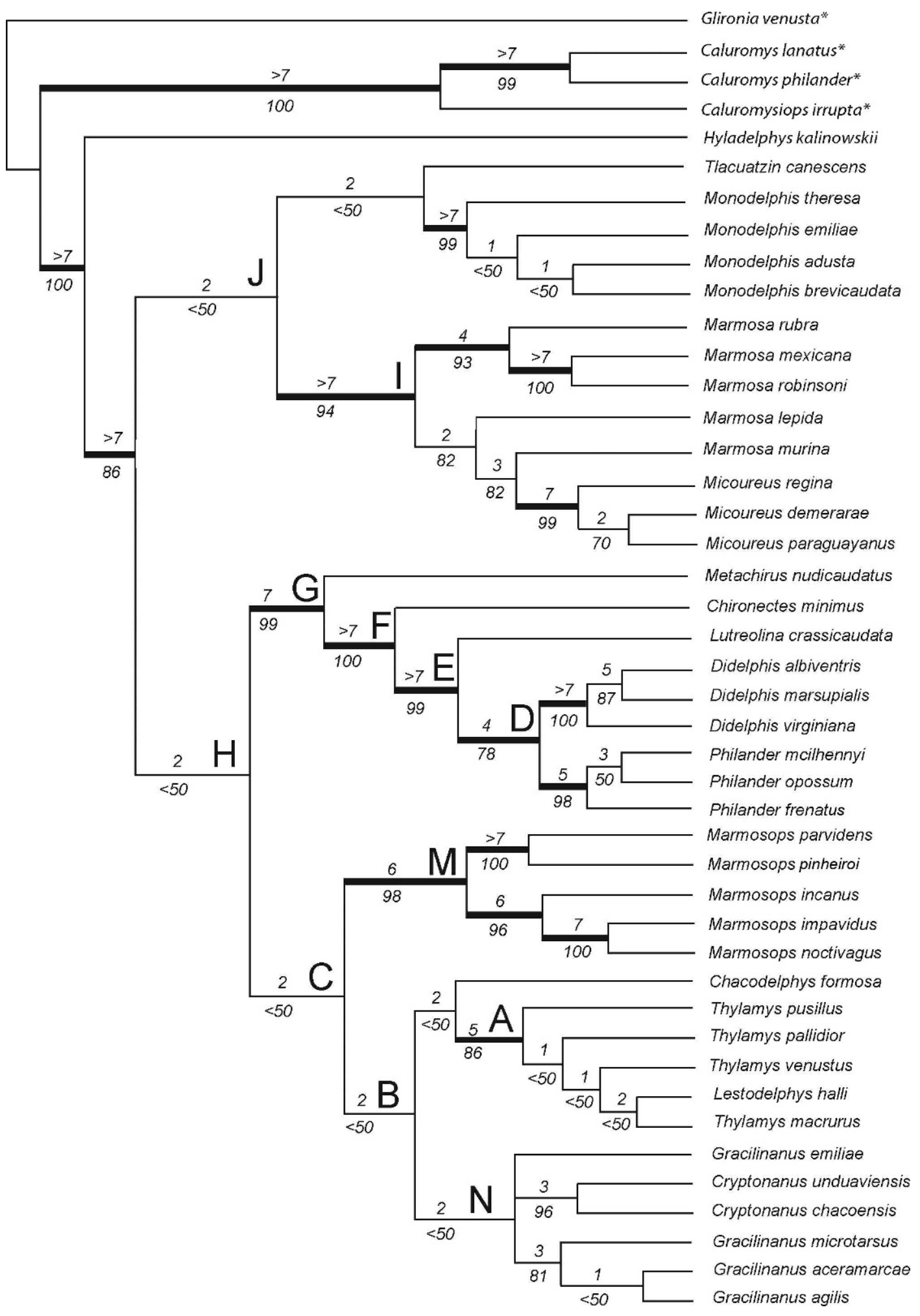


phyletic clade. Another interesting example of a functional component in postcranial characters is the unusual basal position of Lestodelphys on the large opossum clade (node $G$ ) in the TS coding postcranial evidence (fig. 34). The typical position of this terrestrial mouse opossum is close to Thylamys (node A), based on profuse previous evidence (Jansa and Voss, 2000, 2005; Voss and Jansa, 2003; Jansa et al., 2006; Flores, 2003; Creighton, 1984; Reig et al., 1987; Kirsch and Palma, 1995; tables 7, 8). However, Lestodelphys and most of the large opossums shared some character states linked to functional implications on posture and locomotion. In both Lestodelphys and most of large opossums, the spinous process on C6 is laminar (ch. 17[2]) and the femoral lesser trochanter is scarcely developed (ch. 93[0], see Metachirus in fig. 27).

An additional example of an unusual relationship possibly caused by constraints from functional demands is the sister relationship between the terrestrial Metachirus and the specialized swimmer Chironectes in the TS coding postcranial analysis, being closely related to the long recognized monophyletic Didelphis. The previous phylogenetic evidence does not recover Metachirus-Chironectes as sister taxa, with both being successively arranged in the clade containing the large opossums (Reig et al., 1987; Kirsch and Palma, 1995; Jansa and Voss, 2000, 2005; Voss and Jansa, 2003; Gruber et al., 2007). In the scheme obtained from postcranial evidence (fig. 34), both monotypic taxa are clustered as a monophyletic group sharing some synapomorphies associated with specific locomotion or postural patterns: ventral tubercle of atlas of triangular shape (ch. 5[3]), cranial notch of neural arch of axis wide (ch. 15[1], fig. 1), ilium with the distal portion barely curved laterally (ch. 90[1], fig. 26), prepollex absent (ch. 104[0]), and astragalus with well-developed astragalonavicular facet not contacting the sustentacular one (ch. 110[0], fig. 31), although in AMNH 148720 both facets are slightly in contact (see Szalay, 1994: fig. 7.12).

Alternatively, as stated above, the postcranial evidence supports the monophyly of some traditionally already recognized groups (tables 6-8; fig. 33), such as Thylamys, Micoureus, Monodelphis, Marmosops (node M), Didelphis, Philander, and the large opossums (node $\mathrm{G}$ ), which are currently recognized by other kinds of evidence (e.g., Reig et al., 1987; Kirsch et al, 1995; Kirsch and Palma, 1995; Jansa and Voss, 2000, 2005; Jansa et al., 2006; Voss and Jansa, 2003; Gruber et al., 2007; table 8). Similarly, the intermediate position of Hyladelphys among caluromyines and didelphines is also recovered here based on postcranial evidence (figs. 33, 34; table 6), proving its basal position in the didelphid crown group and reinforcing its intermediate phylogenetic position between caluromyines and didelphines already obtained from other sorts of evidence (table 6).

Although not strongly supported, the species of Monodelphis included in this analysis are clustered in a monophyletic group supported by an array of postcranial traits coming from caudal vertebrae morphology, scapula, and some characters from the forelimb and hindlimb. In the same way, Marmosops (node M) is also recovered as monophyletic based on postcranial morphology, although the support values are not high in both TS and CO coding (table 7). This group was already recovered as monophyletic by different kinds of evidence (tables 7, 8); in this report, I add two postcranial characters (deltopectoral crest notably developed, ch. 68[1], and osseous posteroventral extension of the ischium, ch. 86[1]; fig. 23) that reinforce the monophyletic nature of this genus (appendix 3). Other groups of mouse

Fig. 40. Strict consensus of four equally most parsimonious trees resulting from cladistic parsimony analysis of morphological and genetic data combined, excluding RAG-1 sequences, for 44 didelphid taxa where polymorphic data were treated as transformation series (TS). Numbers above branches refer to absolute Bremer support values $(\geq 1)$. Numbers below branches refer to jackknife frequencies (cutoff value $=50 \%$ ). Heavy lines denote branches with a decay index of $\geq 4$. Outgroup taxa are indicated with asterisks. Alphabetic labels indicate didelphine clades discussed in the text. 
opossums previously recognized, such as Thylamys and Micoureus, are also recovered under $\mathrm{CO}$ coding postcranial morphology alone (fig. 33). However, even if both genera where recovered as well-supported natural groups in some published analyses (e.g., Jansa and Voss, 2005; Gruber et al. 2007), their monophyletic condition was proved only in a molecular or combined frame. Here, both groups are slightly supported in a morphological context (appendix 3): in Thylamys the diaphragmatic element is placed on T10 (ch. 27[0]), the longitudinal groove in the lateral surface of the ulna is absent (ch. 77[0]), and the bicipital tuberosity of the radius is scarcely marked (ch. 81[0]), whereas in Micoureus the medial relief for $m$. teres major on the humerus is absent (59[0]).

As mentioned above, the large opossums (Didelphis, Lutreolina, Chironectes, Philander, and Metachirus; node G) were widely recognized in previous papers (table 8). Some postcranial characters, principally traits coming from vertebrae, ribs, and humerus morphology, also add synapomorphies that support the monophyletic condition of this group (appendix 3). A last noncontroversial outcome is the monophyly of Didelphis and Philander, since those genera were largely recovered as natural groups based on several kinds of characters (e.g., Patton et al., 1996; Kirsch et al., 1995; Jansa and Voss, 2003; Flores, 2003; Jansa et al., 2006; Jansa and Voss, 2005; Gruber et al., 2007). The three species of Didelphis included in this report shared the especially strong and scarcely mobile articulation of cervical and thoracic vertebrae (appendix 3), which was illustrated by Coues (1869), whereas the three species of Philander included in this study are clustered by the morphology of the axis, as well as by some special patterns of the forelimb and femur (appendix 3).

\section{CONCLUSIONS}

The recent impulse and increase of knowledge on didelphid phylogeny is the result of the contribution of the inclusion of a denser taxon sampling and the consideration of varied evidence coming from both nuclear sequences (IRBP, DMP-1, and RAG-1) and morphology. Although the nonmolecular aspects frequently have resulted in low resolved topologies compared with hypotheses based on genetic evidence, they are in general agreement with genetic and combined data sets. Topologies coming from postcranial characters only are in general well resolved (mainly in $\mathrm{CO}$ coding analysis) and do not conflict with well-supported groups and relationships (e.g., Hyladelphys as intermediate between didelphines and caluromyines; the monophyly of large opossums; and Didelphis, Philander, Monodelphis, Thylamys, Micoureus, and Marmosops as natural genera), although some unusual clusterings are observed that result from convergences possibly caused by functional demands. However, the contribution of phylogenetic information from postcranial morpohology substantially improves the resolution of previous morphological hypotheses. Some relationships, formerly evidenced only with nuclear sequences, are now recovered with the addition of postcranial characters as well in a morphological framework. Even with the recent consideration of RAG-1 sequences, which reveals some unusual relationships, the phylogenetic information coming from postcranial morphology produces topologies in better agreement with earlier combined hypotheses. However, combined evidence considering postcranial evidence (including and excluding RAG-1 sequences) recovered the clustering of the mouse opossums in the $\mathrm{CO}$ polymorphic character coding. In this sense, the phylogenetic condition of the mouse opossums is still problematic when postcranial characters are considered in a combined data set, although this diverse group is not recovered in other partitioned analyses performed in this report.

The inclusion of new informative sequences and other kinds of morphological characters could provide additional support to groups recognized before or to new topologies. In this sense, the anatomical comparisons on forearm muscles in didelphids and some Australasian taxa performed by Abdala et al. (2006) added some potential new morphological synapomorphies to clades already recognized (i.e., nodes C, G, I, and D). Including a denser taxon sample in such alternative anatomical systems is an important priority in future research on didelphid 
phylogeny for the sake of completeness. Finally, the pending postcranial observations in taxa still not analyzed, as well as the exact condition of missing data of some skeletal traits, could also reinforce several phylogenetic topologies that are slightly supported.

\section{ACKNOWLEDGMENTS}

The financial support for this investigation was provided by a Kalbfleisch Postdoctoral Fellowship of the American Museum of Natural History and partially by CONICET (Consejo de Nacional de Investigaciones Cientificas y Tecnicas, Argentina). I express my appreciation to the Office of Grants and Felloships staff, especially to Diane Bynum and Maria Dickson. At the Department of Mammalogy (AMNH), I thank Pat Brunauer, Rob Voss, Neil Duncan, Darrin Lunde, Teresa Pacheco, John Wahlert, Richard Monk, Robert Anderson, Norberto Giannini, Mariko Kageyama, Valeria Tavares, Ruth O'Leary, Erica Pannen, Adrian Tejedor, Nancy Simmons, and Eileen Westwig for assistance in everything and for kindness during my work at the AMNH. Thanks to Rob Voss, Sergio Solari, Adrián Tejedor, Mónica Díaz, Julián Faivovich, José Tello, Norberto Giannini, Sara Bertelli, and Enrique Penalver for their discussions and suggestions on the earlier version of this work. I am especially grateful to Rob Voss for his trust and support on this project. Thanks to Ines Horovitz, Rob Voss, and an anonymous reviewer for their valuable comments that improved the quality of this work.

Thanks to the curators and staff of museums and institutions for providing valuable assistance on visits and loans: Rubén Bárquez (CML), Daphne Hills (BMNH), Bruce Patterson (FMNH), Jim Patton (MVZ), Mónica Díaz (MMD), Phil Myers (UMMZ), and Hein Van Grow (RMNH). Sharon Jansa kindly provided DMP-1 sequences. Andrea del Moral helped with the English correction. Finally, I also extend my thanks to Luis Luna and Martin Dury for their friendship and support during my days in Westminster (London), and to all my close friends in New York, that is, Sara Bertelli, Adrián Tejedor, Valeria Tavares, Norberto Giannini, Juan Sosa, Enrique
Panalver, Kenny Kozol, Pepe Tello, Marcelo Weksler, Guillermo Cárdenas, Paul Sweet, and Tony Perlstein, for their inestimable help during my unforgettable time there.

\section{REFERENCES}

Abdala, V., S. Moro, and D. Flores. 2006. The flexor tendons in the didelphid manus. Mastozoología Neotropical 13: 193-204.

Alpin, K.P., and M. Archer. 1987. Recent advances in marsupial systematics with a new syncretic classification. In M. Archer (editor), Possums and opossums: studies in evolution., Vol. 1: 15-72. Sydney: Surrey Beatty.

Argot, C. 2001. Functional-adaptive anatomy of the forelimb in the Didelphidae, and the paleobiology of the Paleocene marsupials Mayulestes ferox and Pucadelphys andinus. Journal of Morphology 247: 51-79.

Argot, C. 2002. Functional-adaptive analysis of the hindlimb anatomy of extant marsupials and paleobiology of the Paleocene marsupials Mayulestes ferox and Pucadelphys andinus. Journal of Morphology 253: 76-108.

Argot, C. 2003a. Functional-adaptative anatomy of the axial skeleton of some extant marsupials and the paleobiology of the Paleocene marsupials Mayulestes ferox and Pucadelphys andinus. Journal of Morphology 255: 279-300.

Argot, C. 2003b. Functional adaptations of the postcranial skeleton of two Miocene borhyaenoids (Mammalia, Metatheria), Borhyaena and Prothylacinus, from South America. Palaeontology 46: 1213-1267.

Argot, C. 2004a. Functional-adaptive features and paleobiologic implications of the postcranial skeleton of the late Miocene sabretooth borhyaenoid Thylacosmilus atrox (Metatheria). Alcheringa 28: 229-266.

Argot, C. 2004b. Functional-adaptive analysis of the poscranial skeleton of a Laventan Borhyaenoid, Lycopsis longirostris (Marsupialia, Mammalia). Journal of Vertebrate Paleontology 24: 689-708.

Asher, R.J., I. Horovitz, and M. Sánchez-Villagra. 2004. First combined cladistic analysis of marsupial mammal interrelationships. Molecular Phylogenetics and Evolution 33: 240-250.

Barnett, C.H., and J.R. Napier. 1953. The form and mobility of the fibula in metatherian mammals. Journal of Anatomy 87: 207-213.

Bensley, B.A. 1903. On the evolution of the Australian Marsupialia, with remarks on the relationships of the marsupials in general. Transactions of the Linnean Society of London, Zoology 9: 83-217 + pls. 5-7. 
Bezuidenhout, A.J., and H.E. Evans. 2005. Anatomy of the woodchuck (Marmota monax). Special Publication American Society of Mammalogists 13: 1-180.

Bremer, K. 1994. Branch support and tree stability. Cladistics 10: 295-304.

Campbell, J.A., and D.R. Frost. 1993. Anguid lizards of the genus Abronia: revisionary notes, description of four new species, a phylogenetic analysis, and key. Bulletin of the American Museum of Natural History 216: 1-121.

Clemens, W.A. 1968. Origin and early evolution of marsupials. Evolution 22: 1-18.

Coues, E. 1869. The osteology and myology of Didelphyidae Didelphis virginiana. Memoires of the Boston Society of Natural History 2: 41-154.

Creighton, G.K. 1984. Systematic studies on opossums (Didelphidae) and rodents (Cricetidae). Ph.D. dissertation, University of Michigan, Ann Arbor.

Crompton, A.W. 1989. The evolution of mammalian mastication. In D.B. Wake and J. Roth (editors), Complex organismal function: integration and volution in vertebrates: $23-40$. New York: John Wiley.

Crompton, A.W., and K. Hiiemae. 1970. Molar occlusion and mandibular movements during occlusion in the American opossum, Didelphis marsupialis. Zoological Journal of the Linnean Society 49: 21-47.

Crompton, A.W., and W. Hylander. 1986. Changes in mandibular function following the acquisition of a dentary-squamosal jaw articulation. In N. Hotton III, P.D. MacLean, J.J. Roth, and E.C. Roth (editors), The ecology and biology of mammal-like reptiles: 263-282. Washington, D.C.: Smithsonian Institution Press.

Elftman, H.O. 1929. Functional adaptations of the pelvis in marsupials. Bulletin of the American Museum of Natural History 58(5): 189-232.

Evans, H.E. 1993. Miller's Anatomy of the dog. 3rd ed. Philadelphia: W.B. Saunders.

Filan, S.L. 1990. Myology of the head and neck of the bandicoot (Marsupialia: Peramelemorphia). Australian Journal of Zoology 38: 617-634.

Flores, D.A. 2003. Estudio taxonómico y zoogeográfico de los marsupiales de Argentina. Unpublished Ph.D. dissertation., Universidad Nacional de Tucuman, Argentina.

Flores, D.A., R.M. Bárquez, and M. Díaz. 2008. A new species of Philander Brisson, 1762 (Didelphimorphia, Didelphidae). Mammalian Biology 73: 14-24.

Flower, W.H. 1885. An introduction to the osteology of Mammalia. $3^{\text {rd }}$ ed. London: Macmillan.

Gardner, A. 2005. Order Didelphimorphia. In D.E. Wilson and M. Reeder (editors), Mammals species of the world. 3rd ed.: 3-18. Baltimore, MD: John Hopkins University Press.

Gebo, D.L. 1989. Locomotor and phylogenetic consideration in anthropoid evolution. Journal of Human Evolution 18: 201-233.

Giannini, N.P., and S. Bertelli. 2004. A phylogeny of extant penguins based on integumentary characters. Auk 121: 422-434.

Gilbert, S. 1994. Pictorial anatomy of the cat. 3rd ed. Toronto: University of Toronto Press.

Goin, F.J. 1993. Living South American opossums are not living fossils. Abstracts of the 6th International Theriological Congress: 112-113. Sydney, Australia.

Goin, F.J. 1995. Los Marsupiales. In M.T. Alberdi, G. Leone, and E.P. Tonni (editors), Evolución Biológica y climática en la región pampeana durante los últimos cinco millones de años, un ensayo de correlación con el mediterráneo occidental: 162-179. Madrid: Museo Nacional de Ciencias Naturales.

Goloboff, P., J.S. Farris, M. Källersjö, B. Oxelman, M. Ramírez, and C. Szumik. 2003. Improvements to resampling measures of group supports. Cladistics 19: 324-332.

Goloboff, P., J.S. Farris, and K. Nixon. 2004. T.N.T.: tree analysis using new technologies. Program and documentation. Available in www.cladistics.org.

Goslow, G.E., H.J. Seeherman, C.R. Taylor, M.N. McCutchin, and N.C. Heglund. 1981. Electrical-activity and relative length changes of dog limb muscles as a function of speed and gait. Journal of Experimental Biology 94: 15-42.

Grand, T.I. 1983. Body weight: its relationships to tissue composition, segmental distribution of mass, and motor function. III. The Didelphidae of French Guyana. Australian Journal of Zoology 31: 299-312.

Gregory, W.K. 1910. The orders of mammals. Bulletin of the American Museum of Natural History 27: 1-524.

Gruber, K.F., R.S. Voss, and S.A. Jansa. 2007. Base-compositional heterogeneity in the RAG1 locus among didelphid marsupials: implications for phylogenetic inference and the evolution of GC content. Systematic Biology 56: 1-14.

Herbin, M., V. Jeanne, J.P. Gasc, and P.P. Vidal. 2000. Geometrie du squelette cervical durant la transition repos-locomotion: eneralisation aux caracteristiques du repertoire moteur des rongeurs. Compte Rendues de l'Academie des Sciences Serie III Sciences de la Vie 324: 45-50.

Hershkovitz, P. 1999. Dromiciops gliroides Thomas, 1894, last of the Microbiotheria (Marsupialia), with a review of the Family Microbiotheriidae. Fieldiana Zoology New Series 93: $1-60$. 
Horovitz, I., and M.R. Sánchez-Villagra. 2003. A morphological analysis of marsupial mammal higher-level phylogenetic relationships. Cladistics 19: 181-212.

Howell, A.B. 1965. Speed in animals: their specialization for running and leaping. New York: Hafner Publishing Company.

Jansa, S.A., J.F. Forsman, and R.S. Voss. 2006. Different patterns of selection on nuclear genes IRBP and Dmp1 affect the efficiency but not the outcome of phylogeny estimation for didelphid marsupials. Molecular Phylogenetics and Evolution 38: 363-380.

Jansa, S.A., and R.S. Voss. 2000. Phylogenetic studies on didelphid marsupials. I. Introduction and preliminary results from nuclear IRBP gene sequences. Journal of Mammalian Evolution 7: 43-77.

Jansa, S.A., and R.S. Voss. 2005. Phylogenetic relationships of the marsupial genus Hyladelphys based on nuclear gene sequences and morphology. Journal of Mammalogy 86: 853-865.

Jenkins, F.A., Jr., 1970. Anatomy and function of expanded ribs in certain edentates and primates. Journal of Mammalogy 51: 288-301.

Jenkins, F.A., Jr., and S.M. Camazine. 1977. Hip structure and locomotion in ambulatory and cursorial carnivores. Journal of Zoology 181: $351-370$.

Jenkins, F.A., Jr., and D. McClearn. 1984. Mechanisms of hind foot reversal in climbing mammals. Journal of Morphology 182: 197-219.

Jenkins, F.A., Jr., and W.A. Weijs. 1979. The functional anatomy of the shoulder in the Virginia opossum (Didelphis virginiana). Journal of Zoology 188: 379-410.

Johnson, S.E., and L.J. Shapiro. 1998. Positional behavior and vertebral morphology in atelines and cebines. American Journal of Physical Anthropology 105: 333-354.

Kirsch, J.A.W., and M. Archer. 1982. Polythetic cladistics, or, when parsimony's not enough: the relationships of carnivorous marsupials. In $\mathbf{M}$. Archer (editor), Carnivorous Marsupials., Vol. 2: 595-620. Mosman, NSW: Royal Society of New South Wales.

Kirsch, J.A.W., A.W. Dickerman, and O.A. Reig. 1995. DNA/DNA hibridization studies of Carnivorous Marsupials IV. Intergeneric relationships of the opossum (Didelphidae). Marmosiana 1: 57-78.

Kirsch, J.A.W., and R.E. Palma. 1995. DNA/ DNA hybridization studies of carnivorous marsupials. V. A further estimate of relationships among opossums (Marsupialia, Didelphidae). Mammalia 59: 403-425.

Klima, M. 1987. Early development of the shoulder girdle and sternum in marsupials
(Mammalia, Metatheria). Advances in Anatomy, Embryology and Cell Biology 109: 1-91.

Kurz, C. 2005. Ecomorphology of opossum-like marsupials from the Tertiary of Europe and a comparison with selected taxa. Darmstädter Beiträge zur Naturgeschichte 14: 21-26.

Lanyon, L.E. 1980. The influence of function on the development of bone curvature an experimental study on the tibia rat. Journal of Zoology 192: 457-466.

Larson, S.G. 1993. Functional morphology of the shoulder in primates. In D.L. Gebo (editor), Postcranial adaptation in nonhumans primates: 45-69. DeKalb: Northern Illinois University Press.

Lew, D., R. Pérez-Hernández, and J. Ventura, J. 2006. Two new species of Philander (Didelphimorphia, Didelphidae) from northern South America. Journal of Mammalogy 87: 224-237.

Lunde, D.P., and W.A. Shutt. 1999. The peculiar carpal tubercles of male Marmosops parvidens and Marmosa robinsoni (Didelphidae: Didelphinae). Mammalia 63: 495-504.

Mabee, P.M., and J. Humphries. 1993. Coding polymorphic data: examples from allozymes and ontogeny. Systematic Biology 42: 166-81.

MacLeod, N., and K.D. Rose. 1993. Inferring locomotor behavior in Paleogene mammals via Eigenshape analysis. American Journal of Science 293-A: 300-355.

MacPhee, R.D.E., and L.L. Jacobs. 1986. Nycticeboides simpsoni and the morphology, adaptations, and relationships of Miocene Siwalik Lorisidae. In K.M. Flanagan and J.A. Lillegraven (editors), Contributions to geology: 131161. Laramie: University of Wyoming Press.

Mann-Fischer, G. 1953. Filogenia y función de la musculatura en Marmosa elegans (Marsupialia, Didelphydae). Investigaciones Zoológicas Chilenas 1: 3-15.

Mann-Fischer, G. 1956. Filogenia y función de la musculatura en Marmosa elegans (Marsupialia, Didelphydae). 2 da. Parte. Investigaciones Zoológicas Chilenas 3: 3-28.

Marshall, L.G., J.A. Case, and M.O. Woodburne. 1990. Phylogenetic relationship of the families of marsupials. In H.H. Genoways (editor), Current mammalogy: 433-505. New York: Plenum Press.

Marshall, L.G., and D. Sigogneau-Russell. 1995. Pucadelphys andinus (Marsupialia, Mammalia) from the early Palaeocene of Bolivia. Part 3. The postcranial skeleton. Mémoires du Muséum National d'Histoire Naturelle 165: 91-164.

Martin, K.E.A., and S. Mackay. 2003. Postnatal development of the fore- and hindlimbs in the grey short tailed opossum, Monodelphis domestica. Journal of Anatomy 202: 143-152. 
Maynard Smith, J., and R.J.G. Savage. 1955. Some locomotory adaptations in mammals. Zoological Journal of the Linnean Society 42: 603-622.

Muizon, C. de. 1998. Mayulestes ferox, a borhyaenoid (Metatheria, Mammalia) from the early Paleocene of Bolivia: phylogenetic and paleobiologic implications. Geodiversitas 20: 19-142.

Muizon, C. de, and C. Argot. 2003. Comparative anatomy of the Tiupampa didelphimorphs: an approach to locomotory habits of early marsupials. In M.E. Jones, C.R. Dickman, and M. Archer (editors), Predators with pouches: the biology of carnivorous marsupials: 43-62. Collingwood, VIC: CSIRO Publishing.

Oxnard, C.E. 1963. Locomotor adaptations in the primate forelimb. In J. Napier and N.A. Barnicot (editors), The primates: 165-182. London: Symposium Zoological Society of London.

Patterson, B., and R. Pascual. 1972. The fossil mammal fauna of South America. In A. Keast, F.C. Erk, and B.P. Glass (editors), Evolution, mammals and southern continets: 247-309. Albany: State University of New York Press.

Patton, J.L., S.F. dos Reis, and M.N.F. da Silva. 1996. Relationships among Didelphid Marsupials based on secuence variation in the mitochondrial cytochrome b gene. Journal of Mammalian Evolution 3(1): 1-29.

Pridmore, P.A. 1992. Trunk movements during locomotion in the Marsupial Monodelphis domestica (Didelphidae). Journal of Morphology 211: 137-146.

Reig, O.A., J.A.W. Kirsch, and L.G. Marshall. 1987. Systematic relationships of the living and neocenozoic American "opossum-like" marsupials (Suborder Didelphimorphia), with comments on the classification of these and of the Cretaceous and Paleogene New World and European metatherians. In M. Archer (editor), Possums and opossums: studies in evolution. Vol. 1: 1-89. Sydney: Surrey Beatty.

Roberts, D. 1974. Structure and function of the primate scapula. In F.A. Jenkins (editor), Primate locomotion: 171-200. New York: Academic Press.

Rockwell, H., F. Gaynor Evans, and H. Pheasant. 1938. The comparative morphology of the vertebrate spinal column its form as related to function. Journal of Morphology 63: 87-117.

Sánchez-Villagra, M., S. Ladevèze, I. Horovitz, C. Argot, J.J. Hooker, T. Macrini, T. Martin, S. Moore-Fay, C. de Muizon, T. Schmelzle, and R. Asher. 2007. Exceptionally preserved North American Paleogene matatherians: adaptations and discovery of a major gap in the opossum fossil record. Biology Letters 3: 318-322.

Sanders, W., and B. Bodenbender. 1994. Morphometric analysis of lumbar vertebra UMP 67-28: implications for spinal function and phylogeny of the Miocene Moroto hominid. Journal of Human Evolution 26: 203-237.

Sargis, E.J. 2001. A preliminary qualitative analysis of the axial skeleton of tupaiids (Mammalia, Scandentia): functional morphology and phylogenetic implications. Journal of Zoology 253: 473-483.

Sargis, E.J. 2002. Functional morphology of the hindlimb of tupaiids (Mammalia, Scandentia) and its phylogenetic implications. Journal of Morphology 254: 149-185.

Sears, K. 2004. Constraints on the morphological evolution of marsupial shoulder girdles. Evolution 58: 2353-2370.

Shapiro, L.J. 1993. Functional morphology of the vertebral column in primates. In D. Gebo (editor), Poscranial adaptation in nonhuman primates: 121-149. DeKalb: Northern Illinois University Press.

Shapiro, L.J. 1995. Functional morphology of indrid lumbar vertebrae. American Journal of Physical Anthropology 98: 323-342.

Shapiro, L.J., and W.L. Jungers. 1994. Electromyography of back muscles during quadrupedal and bipedal walking in Primates. American Journal of Physical Anthropology 93: 491-504.

Simpson, G.G. 1971. The evolution of marsupials in South America. Anais da Academia Brasileira de Ciencias 43(Suppl.): 103-118.

Solari, S. 2004. A new species of Monodelphis (Didelphimorphia, Didelphidae) from southeastern Peru. Mammalian Biology 69: 145-152.

Solari, S. 2007. New species of Monodelphis (Didelphimorphia: Didelphidae) from Peru, with notes on M. adusta (Thomas, 1897). Journal of Mammalogy 88: 319-329.

Szalay, F.S. 1982. A new appraisal of marsupial phylogeny and classification. In $\mathrm{M}$. Archer (editor), Carnivorous marsupials: 621-640. Mosman, NSW: Royal Society of New South Wales.

Szalay, F.S. 1994. Evolutionary history of the marsupials and an analysis of osteological characters. New York: Cambridge University Press.

Szalay, F.S., and E.J. Sargis. 2001. Model-based analysis of poscranial osteology of marsupials of Palaeocene of Itaboraí (Brazil) and the phylogenetics and biogeography of Metatheria. Geodiversitas 23: 139-302.

Tardieu, C. 1983. L'articulation du genou analyse morpho-fonctionelle chez les primates et les hominides fossiles. Cahiers de Palèoanthropologie. Paris: Presses du CNRS.

Taylor, M.E. 1974. The functional anatomy of the forelimbs of some African Viverridae (Carnivora). Journal of Morphology 143: 307-336. 
Taylor, M.E. 1976. The functional anatomy of the hindlimbs of some African Viverridae (Carnivora). Journal of Morphology 148: 227-254.

Voss, R.S., A. L Gardner, and S.A. Jansa. 2004. On the relationships of Marmosa formosa Shamel, 1930 (Marsupialia: Didelphidae), a phylogenetic puzzle from the Chaco of northern Argentina. American Museum Novitates 3442: $1-18$.

Voss, R.S., and S. Jansa. 2003. Phylogenetic studies in didelphid marsupials II. Nonmolecular data and new IRBP sequences: separate and combined analyses of didelphine relationships with denser taxon sampling. Bulletin of the American Museum of Natural History 276: $1-82$.

Voss, R.S., D.P. Lunde, and S.A. Jansa. 2006. On the contents of Gracilinanus Gardner and Creighton, 1989, with the description of a previously unrecognized clade of small didelphid marsupials. American Museum Novitates 3482: 1-34.

Voss, R.S., T. Tarifa, and E. Yensen. 2004. An Introduction to Marmosops (Marsupialia: Didelphidae), with the description of a new species from Bolivia and notes on the taxonomy and distribution of other Bolivian forms. American Museum Novitates 3466: 1-40.

Walker, A. 1974. Locomotor adaptations in past and present prosimian primates. In F.A. Jenkins (editor), Primate locomotion: 349-381. New York: Academic Press.

Washburn, S., and J. Buettner-Janush. 1952. The definition of thoracic and lumbar vertebrae. American Journal of Physical Anthropology 10: 251-252.

Weisbecker, V., and M.R. Sánchez-Villagra. 2006. Carpal evolution in Diprotodontian Marsupi- als. Zoological Journal of the Linnean Society 146: 369-384.

Weksler, M. 2006. Phylogenetic relationships of oryzomyine rodents (Muroidea: Sigmodontinae): separate and combined analyses of morphological and molecular data. Bulletin of the American Museum of Natural History 296: 1-149.

White, T.D. 1989. An analysis of epipubic bone function in mammals using scaling theory. Journal of Theoretical Biology 139: 342-357.

White, T.D. 1990. Gait selection in the brush-tail possum (Trichosurus vulpecula), the northern quoll (Dasyurus hallucatus), and the Virginia opossum (Didelphis virginiana). Journal of Mammalogy 71: 79-84.

Whitehead, P.F., W.K. Sacco, and S.B. Hochgraf. 2005. A photographic atlas of physical anthropology. Englewood, CO: Morton Publishing Company.

Wible, J.R. 1990. Petrosals of late cretaceous marsupials from North America, and a cladistic analysis of the petrosal in therian mammals. Journal of Vertebrate Paleontology 10: 183-205.

Wiens, J. 1999. Polymorphism in systematics and comparative biology. Annual Review of Ecology and Systematics 30: 327-362.

Wiens, J.J. 2000. Coding morphological variation withn species and higher taxa for phylogenetic analysis. In J.J. Wiens (editor), Phylogenetic analysis of morphological data: 115-145. Washington, DC: Smithsonian Institution Press.

Wroe, S., M. Ebach, S. Ahyong, C. de Muizon, and J. Muirehead. 2000. Cladistic analysis of dasyuromorphian (Marsupialia) phylogeny using cranial and dental characters. Journal of Mammalogy 81: 1008-1024. 


\section{APPENDIX 1}

\section{Postcranial Material Examined}

The skeletons analyzed for this study are deposited in the following systematic collections, listed in alphabetical order by their acronyms: AMNH, American Museum of Natural History (New York); BMNH, Natural History Museum (London); CML, Colección Mamíferos Lillo (Tucumán, Argentina); FMNH, Field Museum of Natural History (Chicago); MMD, Voucher Collection of Mónica Díaz (will be deposited at the MUSM, Museo de Historia Natural de la Universidad Nacional Mayor San Marcos, Lima, Perú); MSB, Museum of Southwestern Biology (Albuquerque, New Mexico); MVZ, Museum of Vertebrate Zoology (University of California, Berkeley); MZUSP, Museu de Zoologia (Universidade de São Paulo, Brazil); RHMH, Rijksmuseum van Natuurlijke Historie (Leiden, Netherlands); ROM, Royal Ontario Museum (Toronto); RSV, Voucher Collection of R.S. Voss (will be deposited at the AMNH); UMMZ, University of Michigan Museum of Zoology (Ann Arbor); UWZM, University of Wisconsin Zoological Museum (Madison).

Caluromys lanatus: AMNH 133199, 133200, 215001

Caluromys philander: AMNH 95761, 267001, 267002, 267337, 95974, RMNH uncataloged, 12866, 10790, 19646, 20664

Caluromysiops irrupta: AMNH 208101, 244364

Chironectes minimus: AMNH 97319, 148720, 212909, 264571, RMNH uncataloged

Cryptonanus unduaviensis: AMNH 210369, 262401

Didelphis albiventris: AMNH 13102, 148320, 170653, 170654, 170664, 204406, 205301, 205382, 205385, 238006

Didelphis marsupialis: AMNH 13448, 97318, 132784, 209164, 210427, 210428, 210439, 210447, 235003, 255854

Didelphis virginiana: AMNH 70082, 146551, 35908, $235278,70375,21599,215193,240516,240517$, 240519, 240520, 242658

Glironia venusta: MMD 607

Gracilinanus agilis: AMNH 133234, 209157

Gracilinanus microtarsus: MVZ 182057

Hyladelphys kalinowskii: RSV 1572

Lestodelphys halli: UWZM 224223, BMNH 21.6.7.19

Lutreolina crassicaudata: AMNH 133250, 205378, 210421, 210425, CML 2895

Marmosa mexicana: AMNH 189483, 189485, ROM 96090, 99608

Marmosa murina: AMNH 99983, 136159, 254508

Marmosa robinsoni: AMNH 206596, 206597, 206766, 207766, 257209, 257210

Marmosa rubra: FMNH 124612

Marmosops impavidus: AMNH 139226

Marmosops incanus: MVZ 182768, 182769

Marmosops noctivagus: AMNH 136157, 231952

Marmosops parvidens: AMNH 267348, 267344

Marmosops pinheiroi: AMNH 267004, 267005

Metachirus nudicaudatus: AMNH 97320, 136151, 136155, 244617, 267009, CML 7342
Micoureus demerarae: AMNH 257211, 257212, RMNH 12871, 998, 18228

Micoureus regina: AMNH 61391, 148757

Micoureus paraguayanus: CML 7343, 2867, AMNH 42289, 42911

Monodelphis adusta: AMNH 136158, 139227

Monodelphis brevicaudata: AMNH 48133, 257203, RMNH 18079, 12851, 17907

Monodelphis theresa: MVZ 182775

Philander frenatus: MVZ 182066, 182067

Philander mcilhennyi: MVZ 190342

Philander opossum: AMNH 61396, 61864, 97332, 133074, 190446, 210406, 210410, 248703, 254509, 261273, 261276, 262415, RMNH 12835, 12838, 12834, MMD 3737, 1995, 835, 2972

Thylamys macrurus: MZUSP 32094, 32095, 32096, MSB 70700

Thylamys pallidior: AMNH 262405, 262406, 262408, CML 3189, 3192, 3574, 3575

Thylamys pusillus: AMNH 246442, 246446, 275445, 275446, CML 3198, 3573

Thylamys venustus: AMNH 261245, 261253

Tlacuatzin canescens: UMMZ 94604, 94605

\section{APPENDIX 2}

\section{Postcranial Data Matrix} $=$ B.

Caluromys lanatus: 11111112010110002100 $1110000143 \quad 0101 \mathrm{~A} 10111 \quad 00031--200 \quad 2101020110$ $\begin{array}{lllll}1011001102 & 0113101010 & 1100100110 & 1011010110\end{array}$ 01111001110010

Caluromys philander: 11011112010110002100 $1110000243 \quad 010111011100031--200 \quad 2101020110$ $\begin{array}{lllll}1011001102 & 0113101000 & 1100100110 & 1011010110\end{array}$ 01111001110010

Caluromysiops irrupta: 1111101201 0010A02100 $\begin{array}{llll}1110010151 & 01011101110013330200 & 2 \mathrm{~A} 01120110\end{array}$ 00110-1102 $0112102010 \quad 1100101110 \quad 1011010110$ 01111001100010

Chironectes minimus: 11013111011000102101 $11001032010001 \mathrm{~A} 111111113231211 \quad 1000120001$ 10A1110100 0112111001 000000A101 110110A100 01101101100011

Cryptonanus unduaviensis: 00012112011101100000 0A00101342 $1001100111 \quad 0100---010 \quad 1011120110$ $\begin{array}{lllll}1000100011 & 0112101000 & 1000100000 & 1011001110\end{array}$ $110 ? 0000010010$

Didelphis albiventris: $1011100000 \quad 0110002111$ 1110110012 0000A10111 0110-2A1110111011001 11011A0100 110211100010000001001101100110 00111101110010

Didelphis marsupialis: $1011100000 \quad 0110002111$ 1110110012 0000A10111 0110-2A111 01110110A1 11011A0100 110211100010000001001101100110 $001111011 \mathrm{~A} 0010$

Didelphis virginiana: $1011100000 \quad 0110002111$ 1110110012 0000A10111 0110-2A111 0111011001 $110111010011021110001000000100 \quad 1101101110$ 001111011A 0010 
Glironia venusta: 100111110101011011000110101210 $1101100000 \quad-0003--010 \quad 21011201101111001102$ $\begin{array}{llll}0113101000 & 1100100001 & 1011011010 & 0111000110\end{array}$ 0010

Gracilinanus agilis: 0A01311211 110010A000 $010010033210001001110112-201110011110111$ 1AA0100A21 011311A00010000000001011000110 11011000010010

Gracilinanus microtarsus: 0001211211 ??0?100001 0100101311 10001001?? ?112-?0?1? 0011120110 $10001000110113101000 \quad 0000000000 \quad 101100 ? 100$ $11 ? ? ? 000000010$

Hyladeplhys kallinowskii: $00001102001 ? 00001001$ $\begin{array}{lllll}0000103222 & 1001101001 & 1102---110 & 1000120100\end{array}$ $\begin{array}{llll}1010000112 & 0013101010 & 0100000110 & 1011011110\end{array}$ 10111001011010

Lestodelphys halli: $0001211101 \quad 1001102000$ $\begin{array}{lllll}0000102220 & 1000100101 & 0110-11110 & 0110120111\end{array}$ $100010102101120110101100000100 \quad 100100 ? 110$ 01???????? ????

Lutreolina crassicaudata: 10011112011111002000 110010-232 0000111111 1111-2A111 1001120001 $1101110000 \quad 1112111010 \quad 1000101100 \quad 110110 ? 110$ $001 ? ? 101110010$

Marmosa mexicana: 0001210211 1100101001 $\begin{array}{lllll}0110000312 & 1000000111 & 0113021111 & 0011120110\end{array}$ 00001-0010 $0112100000 \quad 0000100100 \quad 1011110110$ 1111100001 A010

Marmosa murina: 0101211201 ??00102001 0100100301 1000A00111 011302A110?011120111 10001A0121 011210A000 00001001001011010110 01011000010010

Marmosa robinsoni: 0A01211201 10A1102000 OA 101013121001 A00111 01A1-2A110 $10011201 \mathrm{A0} \quad 10001 \mathrm{AAA} 210112101000$ 0000100100101110011001111000010010

Marmosa rubra: 110131020111011111000110100211 1000110101 01030--111 ??01100110 1100111112 $\begin{array}{llll}0112101011 & 0000100010 & 101101 ? 110 & 0111100001\end{array}$ 0010

Marmosops impavidus: 00012102011001001001 0A00100302 1000A00111 0110-21110 0111120100 $\begin{array}{lllll}1000100121 & 0112101000 & 1000110100 & 1011100110\end{array}$ $110 ? 1000010010$

Marmosops incanus: 00012102010101001001 01001012111001100110 -110-0AA10 0111120111 $\begin{array}{lllll}1000110111 & 0112111100 & 1000110100 & 1011001110\end{array}$ 11011000010010

Marmosops noctivagus: 0A01210201 100100A001 0100101302 1001A0011A -110-21110 011112A011 $\begin{array}{lllll}1000100111 & 0112101000 & 1000110100 & 1011000110\end{array}$ $110 ? 1000010010$

Marmosops parvidens: 00012102011001101000 $0100101321 \quad 1010100110-110-21110 \quad 1101120101$ $\begin{array}{llll}1000111111 & 0010012110 & 1000110110 & 1011101110\end{array}$ 01011000010010

Marmosops pinheiroi: 0001210211 100110100? 0100101331 101010011A 1110-21110 111112011A $100011011101121010001000110100 \quad 1011101110$ 01011000010010
Metachirus nudicaudatus: 1A01310201 1011102A01 $\begin{array}{lllll}1101100212 & 0010111111 & 1113221111 & 0101011001\end{array}$ $2101110100 \quad 1001011000 \quad 0011001001 \quad 1101101111$ 00101011100100

Micoureus demerarae: 0001111101 1100001A01 $0100100231 \quad 10011001110113021110001112010 \mathrm{~A}$ 100010012B 011310101000001001001011010110 01111000010010

Micoureus paraguayanus: AA01211101 1100001001 $01001002311001100111011302111000111201 \mathrm{AA}$ 1A001A012B 011310A0000000100100 1011010110 01??????? ????

Micoureus regina: 0001111201 100010AA0A $0100100432 \quad 100 \mathrm{~A} 10011101130211100011120100$ 10001000110112101000 0000100A00 1011010110 $01 ? 11000010010$

Monodelphis adusta: 00012112011101101101 $0000100211 \quad 100010011101130100100010110100$ $\begin{array}{lllll}1100110111 & 0112110110 & 0000100100 & 1001001110\end{array}$ 0011?????? ????

Monodelphis brevicaudata: $0101111101 \quad 1101101001$ $\begin{array}{lllll}0101100201 & 1000100111 & 0112-20110 & 0010110100\end{array}$ $\begin{array}{lllll}1100110111 & 0112100110 & 0000100100 & 1001001110\end{array}$ $0011 ? 100010010$

Monodelphis theresa: 00012112011101101001 $0001100342 \quad 1000100111 \quad 01030--010 \quad 1010110100$ $\begin{array}{lllll}1100110111 & 0113101110 & 0000100100 & 1001001110\end{array}$ 00111100010010

Philander frenatus: 1A01211101 1010002100 $\begin{array}{llll}1100101132 & 0000111111 & 1110-21111 & 0101110011\end{array}$ $1101110000 \quad 0113101001 \quad 000000$ A100 1101111110 00111101110010

Philander mcilhennyi: 10011112011000002000 $\begin{array}{llll}1100100233 & 0000110111 & 1110-21111 & 0101110010\end{array}$ $1101110000 \quad 0112001001 \quad 0000000 ? ? 0 \quad 110111 ? 110$ 00011101110010

Philander opossum: 10A1211001 1010001100 $110010013200001101110110-2 A 11101011100 A 1$ $\begin{array}{lllll}1101110000 & 0113111001 & 0000000100 & 1101111110\end{array}$ $00111101110 \mathrm{~A} 10$

Thylamys macrurus: 0001?11201 0101101001 $\begin{array}{lllll}0010100231 & 1000100111 & 1110-00010 & 0011100101\end{array}$ $\begin{array}{lllll}1000100011 & 0112110000 & 0000100100 & 0011001110\end{array}$ 00111000010010

Thylamys pallidior: 00002102111000101001 0A10100321 $1000100111 \quad 0110-20110 \quad 0011121110$ $\begin{array}{lllll}1000100011 & 0112110000 & 0000100100 & 1000001110\end{array}$ 01111000000010

Thylamys pusillus: AA01211211 1101100000 0000100231 $10001001110110-20110 \quad 001112 \mathrm{~A} 110$ 11001A0011 $0112110000 \quad 0000101100 \quad 101100 ? 110$ 01001000010010

Thylamys venustus: 000A210211 100110000A 0A00100321 100010011A -110-20110 0011120100 $10001000110112110000 \quad 0000100 ? 00 \quad 10 ? ? 001110$ 01111000010010

Tlacuatzin canescens: 0000111201 A102100000 $0100102331 \quad 1000100111011332 \mathrm{~A} 0110001120110$ 1000100011011310100000001000010001101100 $010 ? ? 000010010$ 


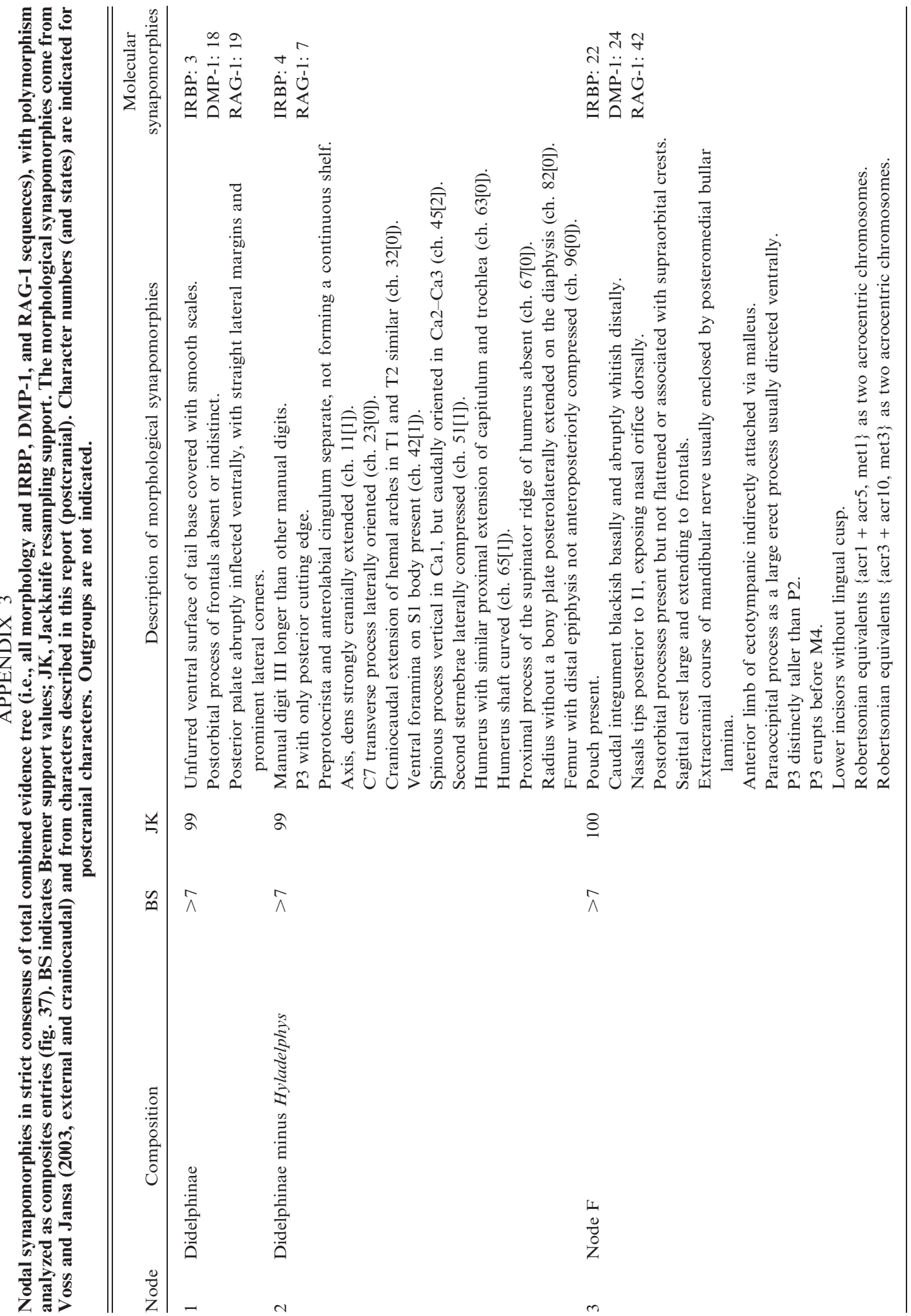




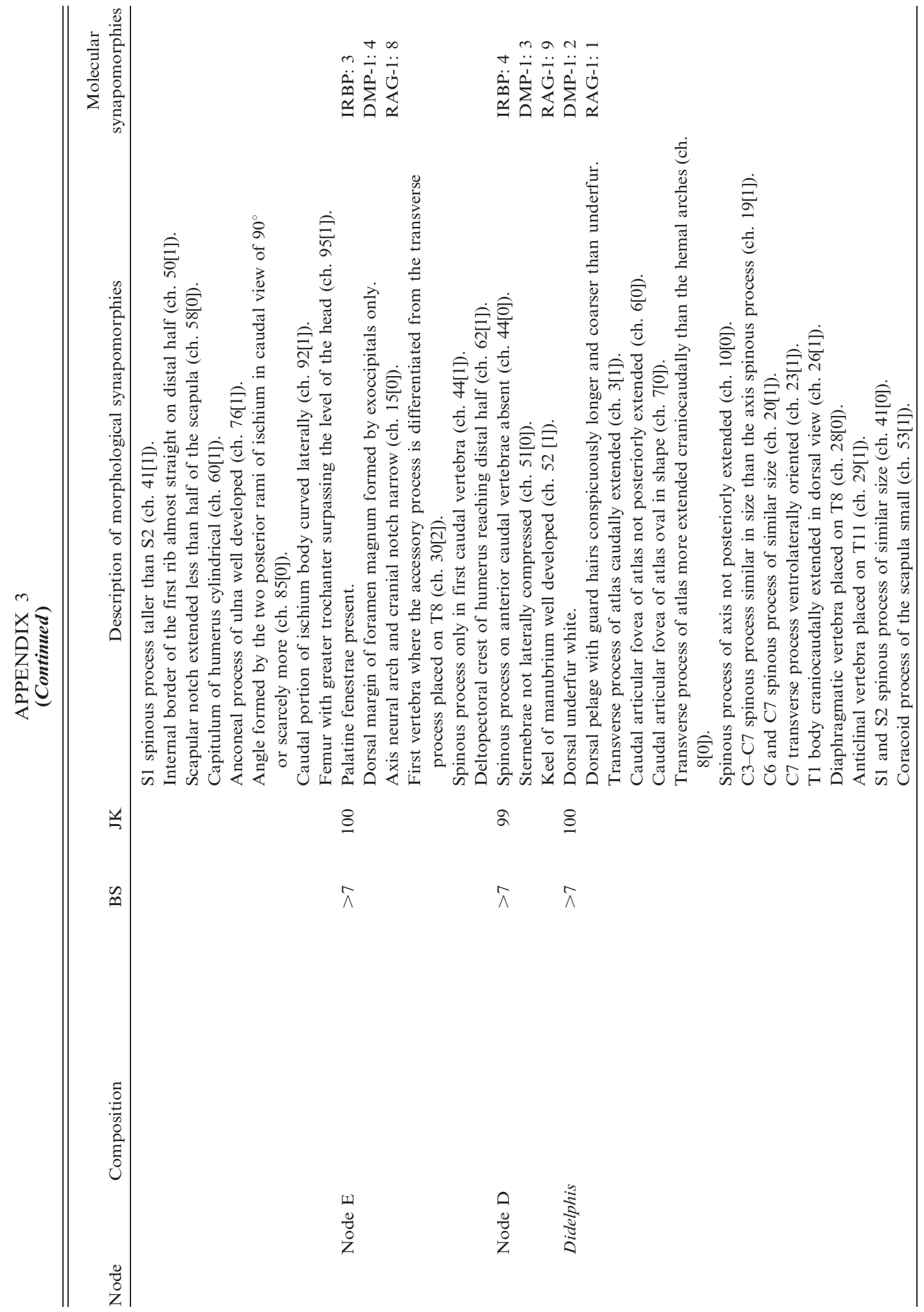




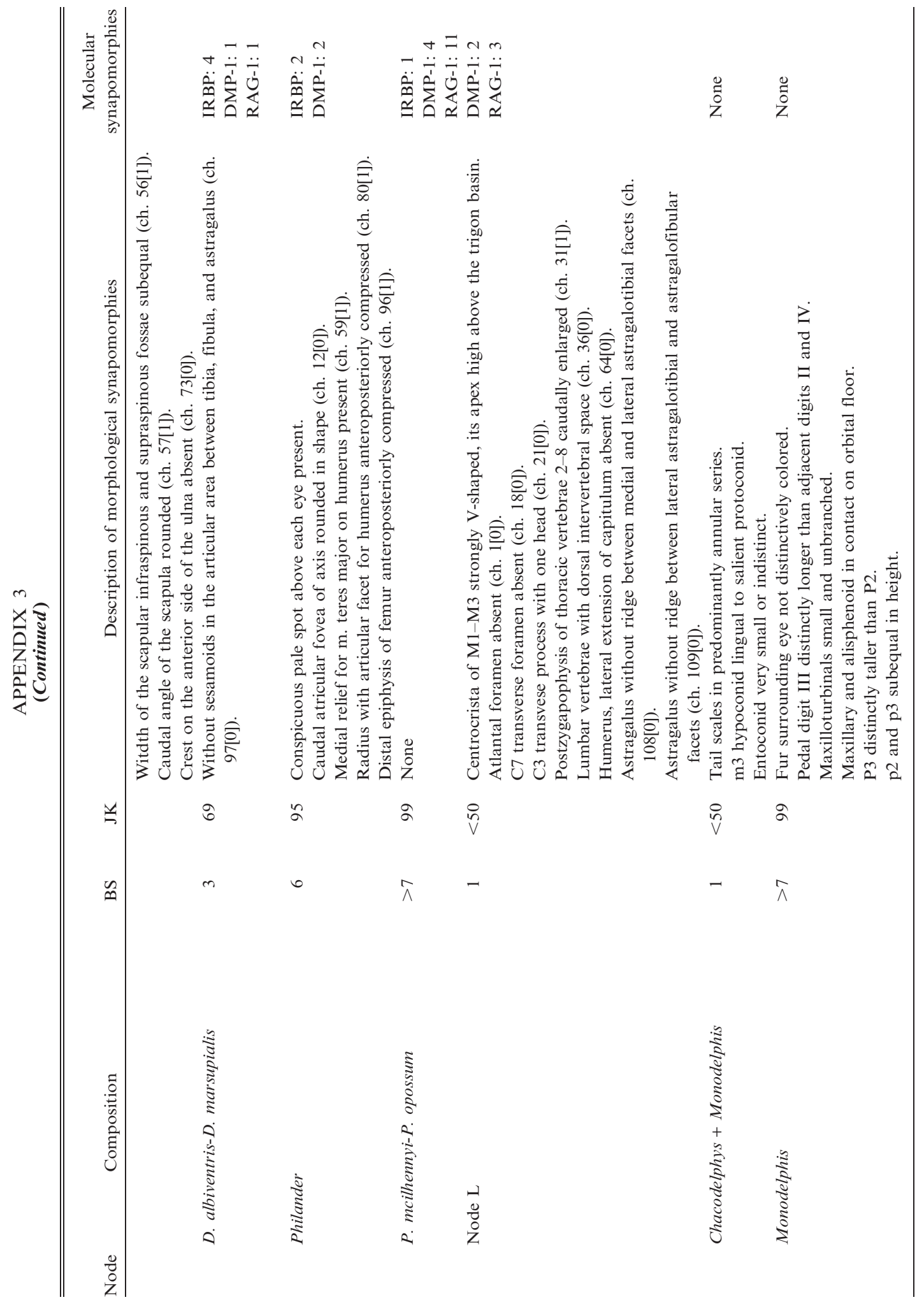




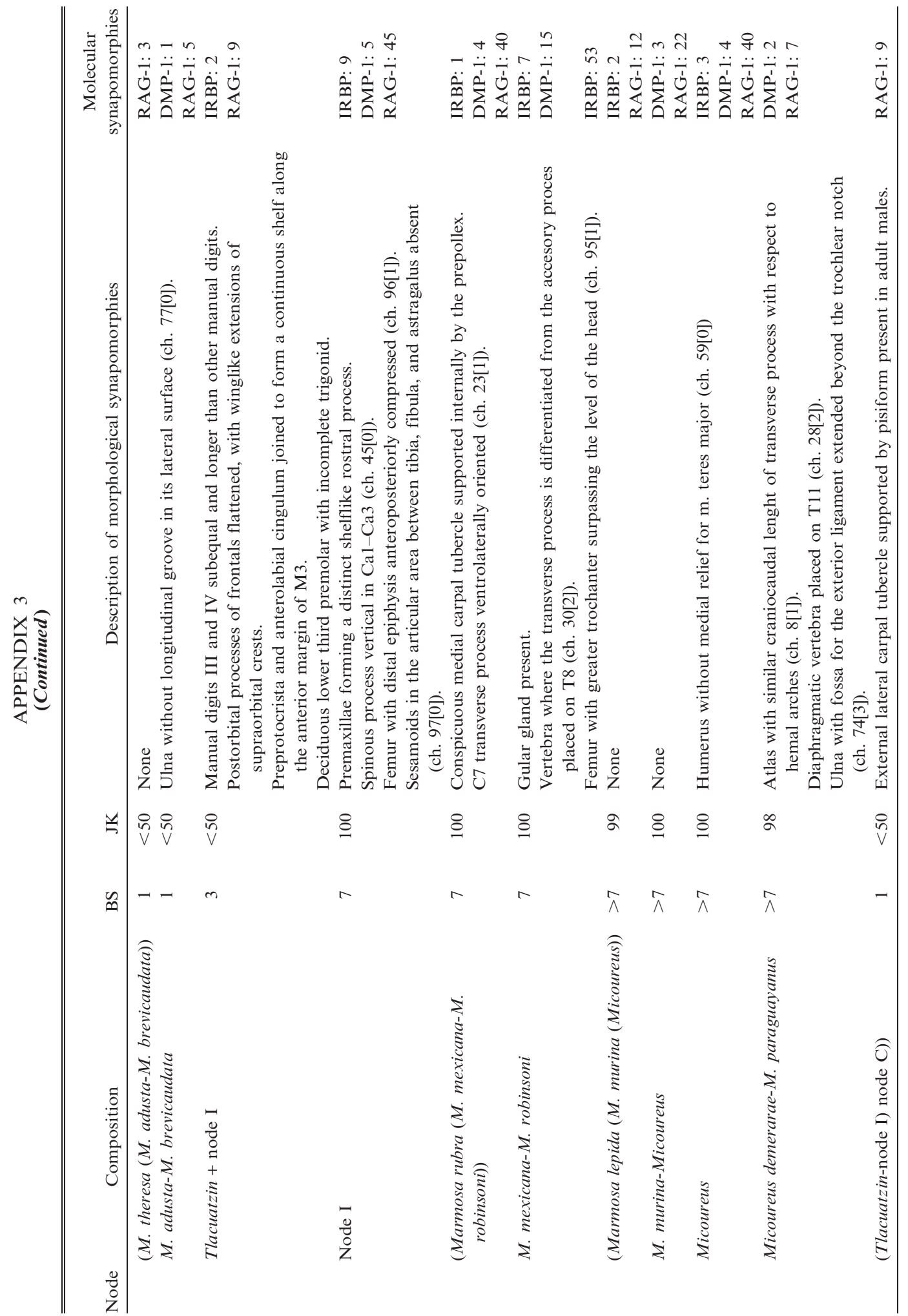




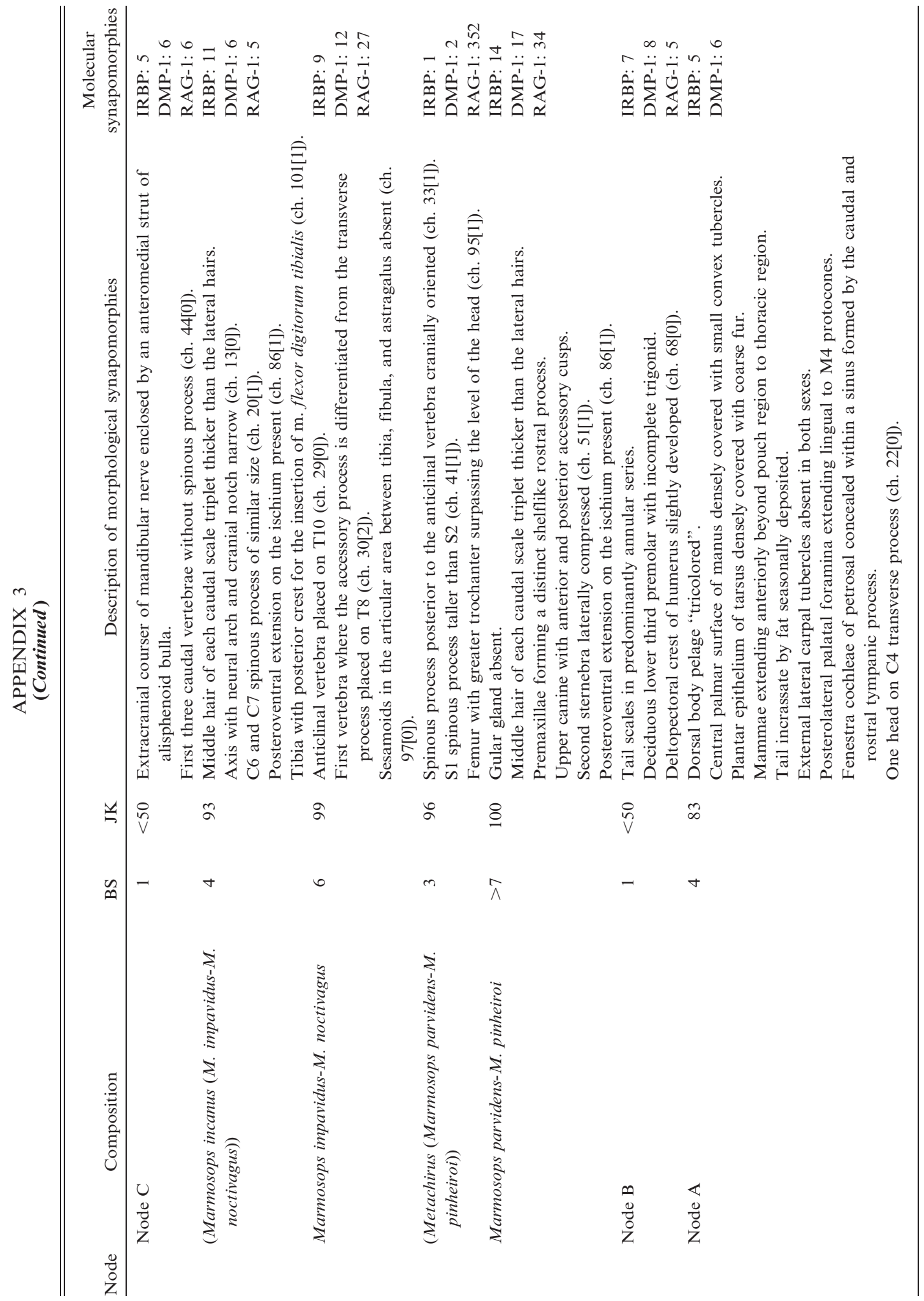




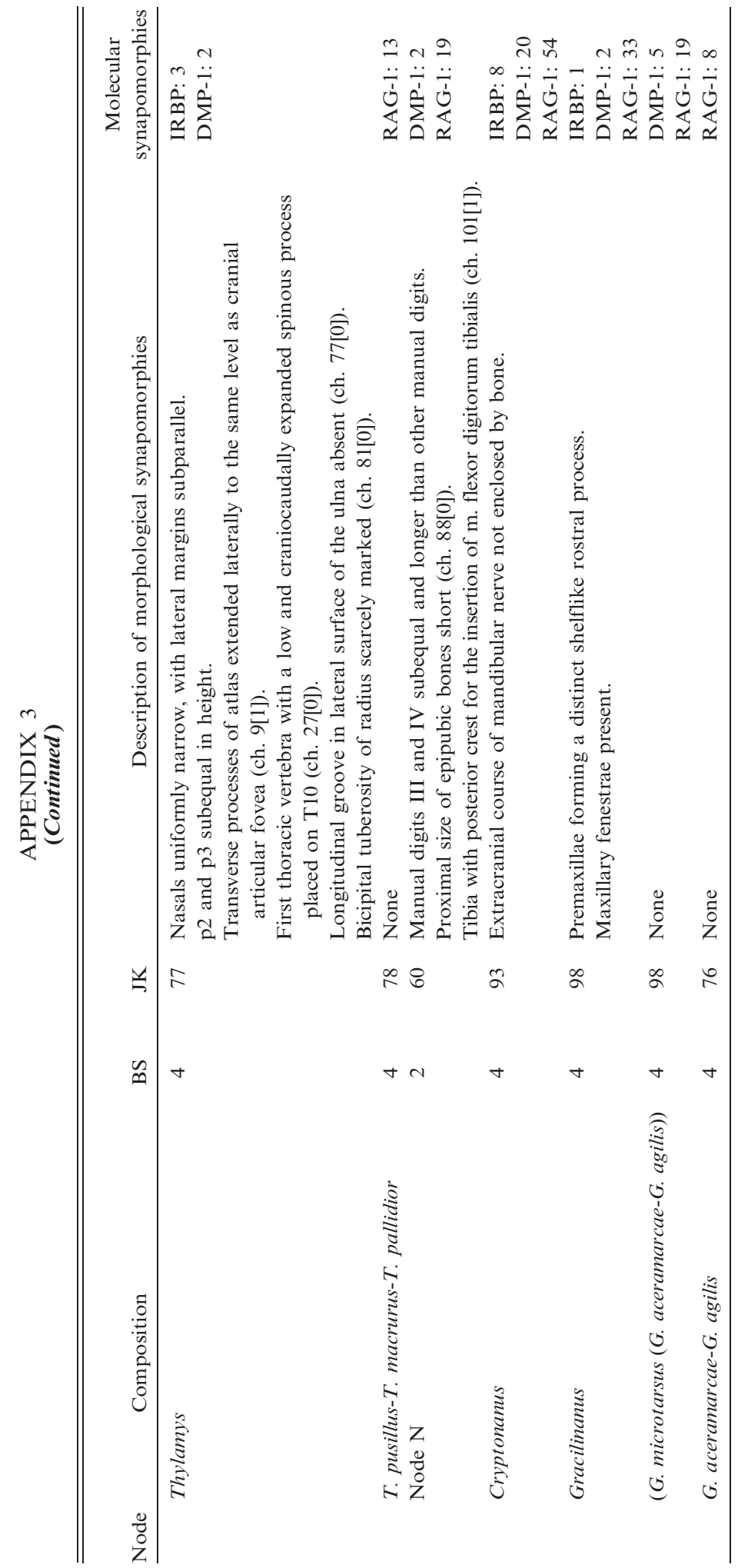

